Accepted to AJ

\title{
The Chemical Compositions of Non-Variable Red and Blue Field Horizontal Branch Stars
}

\author{
Bi-Qing For ${ }^{1}$ and Christopher Sneden \\ Department of Astronomy, University of Texas, Austin, TX 78712, USA
}

\begin{abstract}
We present a new detailed abundance study of field red horizontal branch (RHB) and blue horizontal branch (BHB) non-variable stars. High resolution and high $\mathrm{S} / \mathrm{N}$ echelle spectra of $11 \mathrm{RHB}$ and $12 \mathrm{BHB}$ were obtained with the McDonald $2.7 \mathrm{~m}$ telescope, and the RHB sample was augmented by reanalysis of spectra of 25 stars from a recent survey. We derived stellar atmospheric parameters based on spectroscopic constraints, and computed relative abundance ratios for 24 species of 19 elements. The species include Si II and Ca II, which have not been previously studied in RHB and BHB $\left(T_{\text {eff }}<9000 \mathrm{~K}\right)$ stars. The abundance ratios are generally consistent with those of similar-metallicity field stars in different evolutionary stages. We estimated the masses of the RHB and BHB stars by comparing their $T_{\text {eff }}-\log g$ positions with HB model evolutionary tracks. The mass distribution suggests that our program stars possess masses of $\sim 0.5 M_{\odot}$. Finally, we compared the temperature distributions of field RHB and BHB stars with field RR Lyraes in the metallicity range $-0.8 \gtrsim[\mathrm{Fe} / \mathrm{H}] \gtrsim-2.5$. This yielded effective temperatures estimates of $5900 \mathrm{~K}$ and $7400 \mathrm{~K}$ for the red and blue edges of the RR Lyrae instability strip.
\end{abstract}

Subject headings: stars:abundances - stars: horizontal-branch

\section{Introduction}

Horizontal branch (HB) stars are evolved objects that are fusing helium in their cores

(Hoyle \& Schwarzschild 1955). As low-mass main sequence stars age, they first ascend the

\footnotetext{
${ }^{1}$ biqing@astro.as.utexas.edu
} 
red giant branch (RGB), undergo internal helium-flash (losing some of their mass somewhere along the RGB), and finally take up residence on the HB while they complete their helium consumption. The helium core mass is relatively constant in all types of HB stars $\left(\sim 0.5 M_{\odot}\right)$, but they have a large hydrogen envelope mass range.

HB stars are commonly found in globular clusters (GCs), as well as in field disk and halo populations of our Milky Way. They exhibit a range of photometric colors (or temperatures) which is known as the HB morphology. The distribution can be divided into several groups:

- Red horizontal branch (RHB) stars, which are all HBs cooler than the instability strip (IS).

- RR Lyraes (RR Lyr), named after their prototype. These are variable stars with intermediate temperature and color, located in the IS.

- Blue horizontal branch (BHB) stars, which are hotter than the RR Lyr IS. Their temperatures ranges from $8000-20,000 \mathrm{~K}$, which is also subdivided into HBA $\left(T_{\text {eff }}<10,000 \mathrm{~K}\right)$ and HBB stars $\left(T_{\text {eff }}>10,000 \mathrm{~K}\right.$ ) (Möhler 2004). This division corresponds roughly to $\mathrm{A}$ and $\mathrm{B}$ spectral type. In this paper, we analyze only HBA stars, referring to them collectively as BHB stars.

- Extreme horizontal branch (EHB) stars, which are hotter extension of HB $(20,000-$ $40,000 \mathrm{~K}$ ). These stars often lie below the main sequence in the Hertzsprung-Russell diagram, and thus they are also referred to as hot subdwarfs (see review by Heber 2009).

The assignment of a star to a particular HB group is based on color (or temperature), but the physical cause that determines the position could be affected by multiple parameters. Metallicity, also referred to as the first parameter, was suggested by Sandage \& Wallerstein (1960) to explain the HB morphology as seen in the GCs. Metal-rich clusters have mostly RHB stars and metal-poor clusters have mostly BHB and/or EHB stars.

However, this is not the full story of the HB morphology. Globular clusters that possess similar metallicity often exhibit different HB types. For example, compare the colormagnitude diagrams of M3 vs M13 (see Rosenberg et al. 2000), which clearly indicates that HB morphology is influenced by other parameter(s).

The early study of Searle \& Zinn (1978) suggested that the cluster age could be the second parameter, but later investigation by, e.g, Peterson et al. (1995) and Behr (2003a) argued that stellar rotation could also be a significant contributor. Alternative explanations, such as CNO abundance (Rood \& Seitzer 1981), mixing and helium abundance (Sweigart 
1997), central concentration of the cluster (Fusi Pecci et al. 1993), and Na-O anticorrelation (Gratton et al. 2007) also have been proposed. Lee et al. (1994) demonstrated that various second parameters can produce different HB morphologies. To what extent these potential second parameters influence the variety of observed HB distributions in GCs remains an open question.

Chemical abundance studies of GCs provide ideal laboratories for testing predictions of stellar evolution and nucleosynthesis. Horizontal branch stars are particularly useful for probing several aspects of post-main sequence evolution because they are sensitive to the composition and structure of main sequence stars prior to the exhaustion of their hydrogen fuel (Behr 2003b). Unfortunately, HBs in GCs and stellar streams are faint and as such, hard to observe at high spectral resolution. On the other hand, field horizontal branch (FHB) stars are significantly brighter than cluster stars, and could be useful in many aspects. For example, FHB stars have been used as tracers of Galactic structure (see Wilhelm et al. 1996; Altmann 2000). In addition, field RR Lyrae stars (easy to identify from their variability) yield important information on stellar evolution and pulsation. Their absolute magnitudes and metallicities provide powerful constraints on synthetic HB models (see Cassisi et al. 2004; Demarque et al. 2000).

While FHB kinematics have been widely used to study Galactic structure, their chemical compositions have received scant attention. There are only a handful of detailed abundance studies of FHB stars to date (see Adelman \& Hill 1987; Adelman \& Philip 1990; Lambert et al. 1996). Behr (2003b) conducted a rotational velocity study of FHB stars, with only the derivation of $\mathrm{Mg}$ abundances for all $\mathrm{HB}$ stars. He performed a more extensive chemical abundance study for BHB stars in GCs (Behr 2003a). A recent large survey of FHB stars was carried out by Preston et al. (2006a), but their sample was limited to very metal-poor RHB stars $([\mathrm{Fe} / \mathrm{H}]<-2)$ that were selected from the HK objective-prism survey. Their primary objectives were to investigate any abundance anomalies in these stars, and to derive the fundamental $T_{\text {eff }}$ red edge of the metal-poor RR Lyr IS. They concluded that: (a) FRHB stars generally possess normal enhancements of $\alpha$-elements; (b) there is a [Si/Fe] dependence on $T_{\text {eff }}$ which is unrelated to nucleosynthesis issues; (c) [Mn/Fe] is subsolar; and (d) the $n$-capture elements have large star-to-star relative abundance scatter. They also derived the temperature of the red edge of the metal-poor RR Lyr IS, by interfacing the temperature distributions of field metal-poor RHB and RR Lyr stars with stars of similar metallicities in globular clusters.

In this paper, we present the first detailed abundance study of field RHB and BHB stars that spans an effective temperature range of $4000 \mathrm{~K}$. We explore possible abundance anomalies and their implications on HB evolution. This work potentially can provide a 
different point of views toward understanding HB morphology, and results should aid in application of HB chemical compositions to stellar stream investigations. $\S 2$ describes the target selection and interstellar reddening. The observations and reduction are given in $\S 3$. In $\S 4$ and 5 , we present the line list compilation, equivalent width measurements and analysis methods. The results of individual elemental abundances and evolutionary states of HB stars are given in $\S 6$ and $\S 7$. We discuss the implication of several elemental abundances of our HB samples in $\S 8$. Lastly, we summarize the results of this work in $\S 9$.

\section{Target Selection and Reddening}

The observed targets for this program were selected from Behr (2003b). That paper contains a compilation of known FHB stars that he used for his rotational velocity study. We selected the FHB stars that have $V<11,[\mathrm{Fe} / \mathrm{H}] \leq-1.2$ and $T_{\text {eff }}<9000 \mathrm{~K}$. The temperature restriction was chosen to avoid abundance anomalies due to gravitational settling and diffusion processes that are observed in the hotter BHB stars (e.g, Behr 2003a). RR Lyr stars were deliberately excluded in this program; a companion study of their chemical compositions will be presented in paper II.

We also included metal-poor field red horizontal branch (MPFRHB) stars studied by Preston et al. (2006a) in our program. We did not re-observe the MPFRHB stars, but we analyzed them in a manner consistent with that of the newly observed targets. We refer the reader to the description of target selection and observational details in Preston et al. (2006a). Table 1 gives basic information for our program stars.

Reddening estimates $E(B-V)$ of individual stars were obtained from the NASA/IPAC Extragalactic Database2 (NED) extinction calculator. This technique is based on the Infrared Astronomical Satellite (IRAS) and Diffuse Infrared Background Experiment (DIRBE) measurements of dust IR emission maps of Schlegel et al. (1998) (hereafter SFD). We chose this method in preference to the older Burstein \& Heiles (1982) maps, which are based on H I 21-cm column density and galaxy counts, because the H I maps suffer from the general problem of saturation in the 21-cm line in high extinction regions and have lower spatial resolution than the SFD maps.

Some uncertainties in $E(B-V)$ values estimated from the SFD maps might arise from missing cold dust emission that is not detected by IRAS. In fact, $E(B-V)$ values determined from SFD are probably systematically larger by $\sim 0.02$ mag as compared to

\footnotetext{
2 http://nedwww.ipac.caltech.edu/forms/calculator.html
} 
those of Burstein \& Heiles 1982) (e.g., see comments in Meléndez et al. 2006 and references therein). Burstein \& Heiles (1982) maps are not error free. In fact, their maps contain systematic effect that arises from fluctuations in galaxy count and variation in gas-to-dust ratio. To be consistent and to reduce the degree of systematic effect in our analysis, we only adopted extinctions from SFD maps. To correct these systematic effects of SFD maps, we used a $10 \%$ correction factor as suggested by Meléndez et al.:

$$
c E(B-V)=0.9 E(B-V)-0.01
$$

where $c E(B-V)$ is the corrected $E(B-V)$. We employed the corrected $E(B-V)$ for calculating the photometric $T_{\text {eff }}$, which we used to compare with our independent spectroscopic $T_{\text {eff }}$ values. The details will be given in $§ 5.1$.

\section{Observations and Reductions}

The observations were made with the McDonald $2.7 \mathrm{~m}$ Smith telescope, using the "2dcoudé" cross-dispersed echelle spectrograph. We used this instrument with a $1.2^{\prime \prime}$ slit and in its "cs23-e2" configuration; it gives a 2-pixel resolving power of $R \equiv \lambda / \Delta \lambda \sim 60,000$ with spectra projected onto a Tektronix $2048 \times 2048$ CCD chip with no binning. The total wavelength range is $\sim 3700-8200 \AA$ with complete spectral coverage for $\lambda<5900 \AA$, and with gaps in coverage increasing toward the red. We usually integrated on the target stars for $1.5 \mathrm{hr}$, yielding $\mathrm{S} / \mathrm{N}$ per resolution element of $\sim 70$ near $4000 \AA, \sim 140$ near $5000 \AA$, and $\sim 240$ near $7000 \AA$. The typical seeing for our observing runs varied from $1.5^{\prime \prime}$ to $2.2^{\prime \prime}$. Our observations in 2007-2008 were taken in conjunction with another project, for which we positioned the grating so that more red portion of the spectrum was projected onto the CCD. This resulted in sacrificing some useful blue-spectral echelle orders, which meant that there were fewer lines available for analysis. Optimal spectral coverage was obtained for observing run in 2009.

ThAr comparison lamp exposures were taken at the beginning and the end of each night. We also took the spectra of hot, rapidly rotating, relatively featureless stars throughout the night at different airmasses. These spectra were used to aid in removing telluric features from the spectra of our program stars. Table 2 summarizes the observations and stars that are listed but lack sufficient numbers of detected Fe I \& Fe II lines for stellar parameter estimations were excluded from abundance analysis.

We performed reductions of the spectra with the IRAF 3 ECHELLE package. The raw

\footnotetext{
${ }^{3}$ The Image Reduction and Analysis Facility, a general purpose software package for astronomical data, is
} 
data were bias, flat-field, and scattered-light corrected, then extracted to one-dimensional spectra and wavelength-calibrated in standard fashion. The wavelength calibration arc identification was based on the line list in the IRAF package data file (thar.dat) and the ThAr wavelength table for the 2dcoudé spectrograph (Allende Prieto 2001). The individual wavelength-corrected spectra were then average combined into a single spectrum.

Subsequently, we used the SPECTRE4 (Fitzpatrick \& Sneden 1987) code to normalize the spectra and to remove cosmic rays contamination from the spectral lines. Figure 1 shows typical normalized spectra of RHB and BHB stars. Several of the hotter BHB stars exhibit significant rotational broadening.

\section{Line List and Equivalent Width Measurements}

We compiled an input line list of various elements from previous studies on HB stars (i.e., Preston et al. 2006a.,b; Hubrig et al. 2009; Khalack et al. 2007, 2008; Clementini et al. 1995 \& Lambert et al. 1996). Species such as Si II and Ca II have been included in past HBB studies, but to our knowledge this is the first use of these species for RHB and BHB analysis. Excitation potentials (E.P.) and laboratory oscillator strengths $(\log g f)$ are extracted from various sources, which we cite in Table 3 .

For each star, we measured the equivalent widths (EWs) of unblended atomic absorption lines interactively with SPECTRE. We either adopted the EW value given by fitting a Gaussian to the line profile or by integrating over the relative absorption across a line profile. If a particular line was contaminated by cosmic rays or had an obviously distorted profile (especially lines in BHB stars can be blended with nearby lines due to rotational broadening), we excluded it. Very strong lines on the damping portion of the curve-of-growth (defined as those with reduced widths $\log \mathrm{RW} \equiv \log \mathrm{EW} / \lambda \gtrsim-4.0)$ are relatively insensitive to abundance, and thus were not measured here. After initial trials, we also excluded very weak lines $(\mathrm{EW}<5 \mathrm{~m} \AA)$ because the $\mathrm{EW}$ measurement errors were too large. Since our program stars have a wide range of $T_{\text {eff }}$ and metallicity, the number of lines measured varied considerably. The lines used for each star, along with species, E.P., $\log g f$, its associated references, and measured EWs are listed in Table 3.

We may compare our EW measurements of stars with existing previous studies. Only a

written and supported by the IRAF programming group of the National Optical Astronomy Observatories (NOAO) in Tucson, AZ.

${ }^{4}$ An interactive spectrum measurement package, available at http://verdi.as.utexas.edu/spectre.html 
few high-resolution, detailed chemical abundance investigations of field BHB stars have been conducted to date. The only published iron EW measurements are from Adelman \& Hill (1987) and Adelman \& Philip (1990), which were measured on coudé spectrograms recorded with photographic plates. Figure $2 \& 3$ show the comparison of Fe I \& Fe II EW measurements in four stars. The literature data for the cooler (CS 22951-077) and hotter (CS 22941-027) MPFRHB stars are from Preston et al. (2006a) and those for the two BHB stars (HD 161817 \& HD 109995) are from Adelman \& Hill (1987). Taking the EW measurements difference between Preston et al. (2006a), Adelman \& Hill (1987) and this study (as shown in Figures 2 \& 3), we find: for CS 22951-077, $\triangle \mathrm{EW}=1.3 \pm 0.3 \mathrm{m \AA}, \sigma=2.7 \mathrm{~m} \AA, 82$ lines; for CS 22941-027, $\Delta \mathrm{EW}=1.0 \pm 0.4 \mathrm{~m} \AA, \sigma=2.7 \mathrm{~m} \AA, 37$ lines; for HD 161817, $\Delta \mathrm{EW}$ $=-2.3 \pm 0.8 \mathrm{~m} \AA, \sigma=4.4 \mathrm{~m} \AA, 32$ lines; and for $\mathrm{HD} 109995, \Delta \mathrm{EW}=-2.4 \pm 1.3 \mathrm{~m} \AA, \sigma=5.3$ $\mathrm{m} \AA, 16$ lines. We only compute the $\mathrm{EW}$ difference of lines with $\mathrm{EW}<75 \mathrm{~m} \AA$ in $\mathrm{BHB}$ stars because the larger EW difference in strong lines of HD 161817 is probably due to the different measurement techniques of the two studies. In our case, strong lines were treated by either fitting the damping wing or integrating over the line profile. Since the deviations $(\Delta \mathrm{EW})$ are small, we conclude that our EW measurements are in excellence agreement with others.

\section{Analysis}

Our analysis is based on equivalent width matching and spectrum synthesis. Both methods require a stellar atmosphere model that is characterized by four parameters: effective temperature $\left(T_{\text {eff }}\right)$, surface gravity $(\log g)$, metallicity $([\mathrm{M} / \mathrm{H}])$ and microturbulence $\left(v_{\mathrm{t}}\right)$. We constructed models by interpolation 5 in Kurucz's non-convective-overshooting atmosphere model grid (Castelli et al. 1997). The elemental abundances were derived using the current version of the local thermodynamic equilibrium (LTE) spectral line synthesis code MOOG6 (Sneden 1973). With the exception of iron $\left(\log _{\epsilon}(\mathrm{Fe})=7.52\right)$, this code adopted the solar and meteoritic abundances of Anders \& Grevesse (1989). The details on determining the stellar parameters and methodologies are given in the following subsections.

\footnotetext{
5 The interpolation code was kindly provided by Andrew McWilliam and Inese Ivans.

${ }^{6}$ Available at http://verdi.as.utexas.edu/moog.html .
} 


\subsection{Stellar Parameters}

An initial stellar atmosphere model was created based on the stellar parameters of Preston et al. (2006a) and Behr (2003b). Final model atmosphere parameters were determined by iteration, through spectroscopic constraints: (1) for $T_{\text {eff }}$, that the abundances of individual Fe I lines show no trend with E.P.; (2) for $v_{\mathrm{t}}$, that the abundances of individual Fe I lines show no trend with reduced width $(\log \mathrm{RW})$; (3) for $\log g$, that ionization equilibrium be achieved between the abundances derived from the Fe I and Fe II species; and (4) for metallicity $[\mathrm{M} / \mathrm{H}]$, that its value is consistent with the $[\mathrm{Fe} / \mathrm{H}]$ determination. In the case of $[\mathrm{Fe} / \mathrm{H}]<-2.5$, we adopted $[\mathrm{M} / \mathrm{H}]=-2.5$ for the stellar atmosphere model due to no available models in our grid below this metallicity. Table 4 presents the derived stellar atmosphere model parameters and Fe metallicities of our program stars.

The standard spectroscopic constraints method has drawbacks. In particular, "spectroscopic" gravities derived from ionization balance may be lower than "trigonometric" gravities derived from stellar parallaxes $(\pi)$ or "evolutionary" gravities inferred from HR-diagram positions (see e.g., Allende Prieto et al. 1999). Such mismatches may arise from statistical equilibria that are not well described by LTE. These so-called NLTE effects are mainly due to the additional ionization of neutral-species beyond collisions by UV photons. The problem can increase with decreasing metallicity due to smaller UV line opacities in metal-poor stars. Discrepancies in derived $[\mathrm{Fe} \mathrm{I} / \mathrm{H}]$ and $[\mathrm{Fe} \mathrm{II} / \mathrm{H}]$ are the result: Fe I lines yield lower abundances than do Fe II lines, which are then "corrected" by decreasing assumed gravities in LTE analysis (Thévenin \& Idiart 1999). A full discussion of NLTE effects is beyond the scope of this paper. In the following section, we consider the effects of log $g$ uncertainties on our derived abundances.

We have compared our spectroscopic $T_{\text {eff }}$ 's to those based purely on photometry. We computed photometric temperatures using the metallicity-dependent $T_{\text {eff-color formula of }}$ giants developed by Alonso et al. (1999). These relationships are based on the infrared flux method (IRFM) (Blackwell \& Shallis 1977). We employed only $V-K$ colors for this exercise. In contrast to $B-V$ colors, where blue continua are severely affected by line blanketing, $V-K$ colors are largely insensitive to the choice of metallicity and gravity.

The $(V-K)$ values of our stars, as listed in Table 1, are based on $V_{\text {Johnson }}$ and 2MASS $J$ and $K_{s}$ magnitudes. The calibration curve of Alonso et al. (1999) is based on $(V-K)_{\text {TCS }}$. Therefore, several color transformations were required. We converted these colors to the TCS system in two ways. First, we simply shifted the 2 MASS $K_{s}$ magnitudes to the $K_{\text {TCS }} 7$

\footnotetext{
${ }^{7} K_{\mathrm{TCS}}$ is the broad-band $K$ magnitude in the photometric system developed for the Observatorio del
} 
using Eq. 5(c) of Ramírez \& Meléndez (2005): $K_{T C S}=K_{2 \mathrm{MASS}}-0.014+0.027(J-K)_{2 \mathrm{MASS}}$. The $V_{\text {TCS }}$ magnitudes are essentially equal to $V_{\text {Johnson }}$, thus the $K$ transformation should be

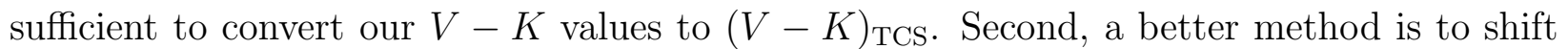
$\left(V_{\text {Johnson }}-K_{s}\right)$ into $(V-K)_{\mathrm{TCS}}$ by two corrections as described in Johnson et al. (2005); we computed the $(V-K)_{\mathrm{TCS}}$ using their Eq. 6: $(V-K)_{\mathrm{TCS}}=0.050+0.993\left(V_{\mathrm{Johnson}}-\right.$ $\left.K_{s}\right)$. For each of these conversion attempts, we then applied extinction corrections to the colors, adopting an extinction ratio of $k=E(V-K) / E(B-V)$, where $k=2.74$ for $(V-K)_{\mathrm{TCS}}\left(\right.$ Ramírez \& Meléndez 2005). Photometric $T_{\text {eff }}$ were subsequently calculated using polynomial relation described in Eq. 8 of Alonso et al. (1999). There are two BHB stars that possess $V-K$ colors that are smaller than $V-K$ range $(<0.2)$ of this equation's calibration. For these stars we simply assumed that the polynomial fit could be extrapolated to $V-K \simeq 0$.

We compared the calculated photometric $T_{\text {eff }}$ of both methods and found that the difference is small $\left(\Delta T_{\text {eff }}=54 \pm 1 \mathrm{~K}, \sigma=6 \mathrm{~K}, N_{\text {star }}=34\right)$ for RHB stars and somewhat larger $\left(\Delta T_{\text {eff }}=109 \pm 3 \mathrm{~K}, \sigma=11 \mathrm{~K}, N_{\text {star }}=11\right)$ for BHB stars. The larger difference for BHB stars is most likely due to the color- $T_{\text {eff }}$ transformation, because it is based mostly on cooler stars. The error of calculated photometric $T_{\text {eff }}$ depends on the slope of the polynomial fit, $\Delta T_{\text {eff }} / \Delta X$, where $\Delta X$ is a function of extinction ratio $(k)$ and error in reddening $(\Delta E(B-V))$. The error is represented by $17 \mathrm{~K}$ per $0.01 \mathrm{mag}$ for $V-K<2.2$ (Alonso et al. 1999).

We show the comparison of the calculated photometric $T_{\text {eff }}$ values that are adopted from the first color-transformation method to the derived spectroscopic $T_{\text {eff }}$ values in Figure 4 . Taking the difference (our spectroscopic $T_{\text {eff }}$ minus photometric $T_{\text {eff }}$ ), we show that both $T_{\text {eff }}$ values of both $\operatorname{RHB}\left(\Delta T_{\text {eff }}=-73 \pm 30 \mathrm{~K}, \sigma=177 \mathrm{~K}, N_{\text {star }}=34\right)$ and BHB stars $\left(\Delta T_{\text {eff }}=59 \pm 91 \mathrm{~K}, \sigma=300 \mathrm{~K}, N_{\text {star }}=11\right)$ are in good agreement.

Ideally our spectroscopic gravities should be compared with trigonometric or physical gravities, but such an exercise is not possible here. Our stars have no reliable parallax data from Hipparcos (Perryman et al. 1997); they are too distant. Most stars selected from the Behr (2003a) catalog have large errors in their parallaxes, and no parallaxes have been reported for stars selected from Preston et al. (2006a).

Teide (Tenerife) $1.5 \mathrm{~m}$ telescope (Alonso et al. 1994). 


\subsection{Parameter Uncertainties}

To estimate the effects of uncertainties in our spectroscopically-based $T_{\text {eff }}$ on derived abundances, we varied the assumed $T_{\text {eff }}$ 's of HD 119516 (RHB) and HD 161817 (BHB). For HD 119516, raising $T_{\text {eff }}$ by $150 \mathrm{~K}$ from the derived $5400 \mathrm{~K}$ produced an unacceptably large trend of derived $\log \epsilon(\mathrm{Fe})$ with excitation potential. For the BHB star, HD 161817, $T_{\text {eff }}$ can be raised to $200 \mathrm{~K}$ before the trend of $\log \epsilon(\mathrm{Fe})$ with E.P. becomes too large. Repeating these trials for other stars suggested that $150 \mathrm{~K}$ and $200 \mathrm{~K}$ are typical uncertainties for the RHB and BHB stars, respectively. The difference between the two groups is due to the lesser number of available Fe I lines in BHB spectra, which causes larger error in $T_{\text {eff }}$ derivation.

We estimated $v_{\mathrm{t}}$ uncertainties in a similar manner, assessing the trends of Fe I abundances with $\log (\mathrm{RW})$. This yielded $v_{t}$ errors of $0.2 \mathrm{~km} \mathrm{~s}^{-1}$ and $0.3 \mathrm{~km} \mathrm{~s}^{-1}$ for RHB and BHB stars, respectively. Finally, (assuming that $\log g$ based on the neutral/ion ionization balance of Fe abundance is correct) from the dependence Fe II abundances with $\log g$, we estimated the error of $\log g$ to be $2 \sigma$ of Fe II abundance error. The mean error of log $g$ to be $\sim 0.16$ dex. We adopted the internal error $(\sigma)$ of Fe I abundances as the model $[\mathrm{M} / \mathrm{H}]$ error.

\subsection{Comparisons with Previous Studies}

We compared our derived $\log g$ and $T_{\text {eff }}$ values with those of Preston et al. (2006a) and Behr (2003b), as shown in Figures 5 \& 6. Behr (2003b) derived these quantities by comparing the synthetic photometric color and the observed color over a grid of $T_{\text {eff }}-\log g$ values. Preston et al. (2006a) employed the same method as we do, i.e., from spectroscopic constraints, but they used both Fe and Ti abundances for determining log $g$ from ionizationbalance considerations. We decided here not to use $\mathrm{Ti}$ in the $\log g$ estimation, because the Ti I $\log g f$ values from the NIST atomic transition database 8 are of relatively high uncertainty and there are not many measurable Ti I lines $(N<6)$ in most cases for our RHB stars. Using small number of lines would cause larger error in log $g$ estimation and could yield systematic error (see below). Additionally, we have no detections of Ti I lines in our BHB sample. Therefore to be consistent in our RHB and BHB star analyses, we decided to only use Fe I and Fe II abundances in estimations of $\log g$.

Our $T_{\text {eff's for }}$ RHB stars are $\Delta T_{\text {eff }}($ Preston-us $)=59 \pm 20 \mathrm{~K}(\sigma=100 \mathrm{~K}, N=25)$ and $\Delta T_{\text {eff }}($ Behr-us $)=154 \pm 40 \mathrm{~K}(\sigma=134 \mathrm{~K}, N=11)$, which are in good agreement. Comparison of BHB stars can only be made with Behr. Our $T_{\text {eff }}$ values generally agree with

\footnotetext{
${ }^{8}$ National Institute of Standards and Technology (NIST): http://www.nist.gov/physlab/data/asd.cfm.
} 
his, $\Delta T_{\text {eff }}$ (Behr-us $)=-152 \pm 43 \mathrm{~K}(\sigma=134 \mathrm{~K}, N=10)$ except for HD 8376 and possibly HD 93329. Our derived RHB log $g$ values are systematically lower ( $\Delta \log g$ (Preston-us) $=0.41 \pm 0.06 \mathrm{dex}, \sigma=0.3 \mathrm{dex}, N=25)$ than those of Preston et al., which is due to different derivation methods. To demonstrate such systematic effect, we performed tests using both Fe and Ti lines. Abundances of neutral species of Titanium is generally larger than ionized species by $0.12-0.2$ dex. As such, this requies a larger log $g$, which is $0.2-0.5$ dex, to achieve the ionization equilibrium for Ti.

Our derived log $g$ values show no correlation with Behr's, and we note significant deviations for HD 8376, HD 6461 and HD 6229. For HD 6461 our derived $[\mathrm{Fe} \mathrm{I} / \mathrm{H}]$ is +0.6 dex higher than Behr's, which in turn forces a larger log $g$ to achieve the ionization equilibrium. Our $T_{\text {eff }}$ for HD 8376 is about $500 \mathrm{~K}$ larger than Behr's estimate, which forces a much larger $\log g$ value in our analysis. We do not have an explanation for the $\log g$ deviation of HD 6229.

\subsection{Microturbulence vs Effective Temperature}

We plot our $v_{\mathrm{t}}$ values against $T_{\text {eff }}$ in Figure 7 , where the correlations (dashed lines) were derived by fitting linear least squares regression lines to the RHB and BHB data. The clear positive correlation of microturbulent velocity with temperature in RHB stars has been found by others (see Preston et al. 2006a and references therein). It is possible that the BHB stars have an anti-correlation between these two quantities. The star-to-star scatter is large, but if we exclude HD 83769, the anti-correlation remains. We have extended the dashed lines beyond their intersection in the figure; comparison of these lines with the RR Lyr data indicates that there is no $v_{\mathrm{t}}$ correlation with $T_{\text {eff }}$ in this doman. This issue will be revisited in paper II.

These trends in derived $v_{\mathrm{t}}$ with $T_{\text {eff }}$ undoubtedly are related to the envelope/atmosphere instabilities of RR Lyr stars. The evolutionary track of a HB star indicates that it evolves from the hot end, crosses the RR Lyr IS into the cool HB region, before ascending to the AGB. As an HB star evolves toward the RR Lyr IS blue edge, its atmosphere begins to be unstable, which results in increasing line widths that we model as increasing microturbulence. And as the HB star evolves away from the RR Lyr IS red edge, the line widths decrease as the stability is regained. We caution here that our microturbulence values are simple compensations for complex physical changes that are occurring in HB stars near the

\footnotetext{
${ }^{9}$ Our derived $v_{\mathrm{t}}$ for HD 8376 is rather uncertain because no $v_{\mathrm{t}}$ choice can eliminate the trend of log $\epsilon(\mathrm{Fe})$ with $\log (\mathrm{EW} / \lambda)$ for this star. This is the only program star for which we have trouble in finding an acceptable $v_{\mathrm{t}}$ value.
} 
instability strip, and thus should be interpreted with caution.

\section{Chemical Abundances}

With the model atmosphere parameters listed in Table 4, we derived the abundances of most elements from their EW measurements. In the cases of Ca II, Mn I, Ni II, Sr II, Zr II, Ba II, La II, and Eu II, the detectable transitions are complex: they are either partially blended, or have significant hyperfine and/or isotopic substructure, or all of these things. We employed spectrum synthesis to determine abundances for these species. That is, for each line we computed theoretical spectra of a wavelength region within $\pm 10 \AA$ of the line for a variety of assumed abundances, then broadened the computed spectrum with Gaussian line profile (or a combination of Gaussian plus rotational velocity line profile), and finally compared these spectra to the observed ones. The assumed abundances were changed iteratively to obtain acceptable synthetic/observed spectrum matches. For stars with detectable rotational line broadening, we began with the $v \sin i$ estimates of Behr (2003b) and derived the final $v \sin i$ based on the fit to observed line profile. Our final numbers were always in good agreement $\left(\Delta v \sin i \simeq 1-2 \mathrm{~km} \mathrm{~s}^{-1}\right)$ with initial values. The damping constant of Barklem \& O'Mara (1998) was adopted whenever possible in both EW analyses and spectrum syntheses method.

We present the derived abundances ratio $[\mathrm{X} / \mathrm{Fe}]$ in Tables 58, and plot these as functions of metallicity in Figure 8,10 and $T_{\text {eff }}$ in Figures 11 13. Non-LTE corrections have been applied to the data on these figures and tables wherever applicable. The mean $[\mathrm{X} / \mathrm{Fe}]$ values of RHB and BHB stars are summarized in Table 9. In the following subsections we comment on individual elements.

The total error in the abundances is a combination of internal error (line-to-line scatter), and external errors (induced by stellar model atmosphere parameter uncertainties). The lineto-line scatter is given by the abundance standard deviation $(\sigma)$ from individual spectral lines. To estimate the errors caused by model parameter uncertainties, we performed numerical experiments for four stars, in which we varied the model parameter errors as estimated in §5.2. These stars are CS 22898-043 (very metal-poor), HD 25532 (moderately metalpoor), HD 93329 (BHB) and $\mathrm{BD}+18^{\circ} 2890$ (RHB). They were selected because they are representative of our whole sample. The results of $[\mathrm{X} / \mathrm{Fe}]$ sensitivity to different stellar model atmosphere parameter variations are shown in Table 10 \& [1], In most cases $\Delta[\mathrm{X} / \mathrm{Fe}] \lesssim 0.05$ in response to changes in $\log g,[\mathrm{M} / \mathrm{H}]$ and $v_{\mathrm{t}}$. On the other hand, varying $T_{\text {eff }}$ by $150 \mathrm{~K}$ has a larger effect on the abundance ratios of cool, metal-poor RHB star BD $+18^{\circ} 2890$, especially on the neutral species. The overall average variations in $[\mathrm{X} / \mathrm{Fe}]$ are small, $\simeq 0.05$. Thus, in general external error from stellar model atmosphere parameters do not greatly influence 
the derived abundance ratios. For abundances derived from one spectral line, we adopted an error of 0.2 dex, judging from the statistical source of error (ie., sensitivity of $\Delta[X / F e]$ with stellar parameters error, uncertainties in measuring the EW or matching a synthetic spectrum etc).

\subsection{The Light Alpha Elements: Magnesium, Calcium and Titanium}

It has been known for decades that metal-poor stars are generally overabundant in $\alpha$ -

elements (e.g., Wallerstein et al. 1963). Our HB stars show standard enhancements in these elements, with neutral species $<[\mathrm{Mg}, \mathrm{Ca}, \mathrm{Si}, \mathrm{Ti} / \mathrm{Fe}]>\simeq+0.3$ (see Figure 8).

Two RHB stars, BD $+18^{\circ} 2890$ and CS 22883-037, exhibit relatively low $[\mathrm{Mg} / \mathrm{Fe}]$, but not in other $\alpha$-elements. Only a single $\mathrm{Mg}$ I line was analyzed in both of these cases, which resulted in larger abundance uncertainties. Caution is advised in interpreting the $\mathrm{Mg}$ abundances of $\mathrm{BD}+18^{\circ} 2890$ and CS 22883-037.

The Calcium abundances of BHB stars have a larger scatter than the RHB stars. There is also an offset, $\sim 0.3$ dex of mean $[\mathrm{Ca} / \mathrm{Fe}]$ of RHB and BHB stars. We investigated this offset by synthesizing the Ca II $3933 \AA \mathrm{K}$-line of BHB stars. This line is rarely used in abundance analyses, as it is extremely strong in cool stars. In our case, the K-line could be analyzed in BHB stars, in which the line is not very strong and uncontaminated in most cases. There are weak interstellar contamination for HD 2857 and BD+252602. However, it does not affect our abundances derivation, which is based on a Gaussian line profile fitting to the line. The abundances in BHB stars for Ca I and Ca II are approximately consistent with each other. The presence of the BHB/RHB offset is currently unknown. We also note that there is an unexplained trend of decreasing $[\mathrm{Ca} / \mathrm{Fe}]$ with increasing $T_{\text {eff }}$ for BHB stars (see Figure 11). Investigation of larger sample of BHB stars might resolve this puzzle.

There are no Ti I lines detectable in our BHB stars. Additionally, our $\log g f$ values for the Ti I lines are taken from the NIST compilation, but their estimated uncertainties are large. In the RHB stars, Ti I lines are visible, but not many measurable lines. The analysis yields a trend of increasing [Ti I/Fe] with increasing $T_{\text {eff }}$ (see Figure 11). This trend is opposite the sense of Si (discussed below) and has been noted by others (see Lai et al. 2008 and references therein). The abundance ratios derived from Ti II, unlike those of the other $\alpha$-elements, do not decline as the metallicity increases. The mean value is flat, with small scatter, across the entire metallicity range. The Ti II-based titanium abundances should be trustworthy as many Ti II lines were used to determine the abundances. 


\subsection{The Alpha Element Silicon: A Special Case}

Substantial dependence of $[\mathrm{Si} \mathrm{I} / \mathrm{Fe}]$ with temperature has been found in previous studies of metal-poor field stars (see Cavrel et al. 2004, Cohen et al. 2004, Preston et al. 2006a, Sneden \& Lawler 2008 \& Lai et al. 2008). This effect seems to depend entirely on $T_{\text {eff }}$; there is no apparent trend with log $g$. To address this puzzle, Shi et al. (2009) investigated NLTE effects in warm metal-poor stars. They showed that the Si I $3905.53 \AA$ lines and Si II $6347 \AA$, $6371 \AA$ Aines exhibit significant NLTE departures in warm metal-poor stars. Their study was limited to a sample of metal-poor dwarfs and a single cool giant. Observationally however, warmer FRHB stars $\left(6000 \mathrm{~K} \lesssim T_{\text {eff }} \lesssim 6400 \mathrm{~K}\right)$ have similar Si abundances to those of metalpoor main sequence turnoff stars, $[\mathrm{Si} / \mathrm{Fe}] \simeq 0$ (see Figure 10 of Preston et al. or Figure 8 of Sneden \& Lawler), in spite of their large gravity differences $(<\Delta \log g\rangle \sim 2)$. Thus, the effect seems to be most dependent on $T_{\text {eff }}$, so we assume that the predicted NLTE effects for main sequence stars will also affect our low gravity, metal-poor, warm RHB and BHB stars. Taking the offsets of +0.1 dex and -0.1 dex to the Si I and Si II abundances from these lines, as suggested by Shi et al., we corrected the abundances of these two species in our program stars with $T_{\text {eff }} \geq 6000 \mathrm{~K}$. Note that there is a large star-to-star scatter for RHB and BHB stars even after this adjustment (see Figure 11). This suggests, in agreement with the conclusions of Shi et al., that addition of an offset is inadequate to produce abundance consistency for this species.

The Si I abundances of all the BHB stars and the CS stars, with the exception of CS 22940-070, were exclusively derived from the 3905.53 $\AA$ line. As always, the reader is cautioned about the abundances derived from a single line. The blue-spectral region of hot stars are not overcrowded with lines, so blending is not an issue in this case. For cool stars, 3905.53 A might be blended with a weak CH transition (Cohen et al. 2004) which would become stronger with decreasing temperature. However, Preston et al. (2006a) argue that the $\mathrm{CH}$ contamination in metal-poor RHB stars is very weak, and will not seriously affect the derived Si abundance. The line is thus essentially ublended and weak enough for abundance determinations in all BHB stars, and in RHB stars with $T_{\text {eff }} \geq 5400 \mathrm{~K}$ and $[\mathrm{Fe} / \mathrm{H}] \leq-2.10$

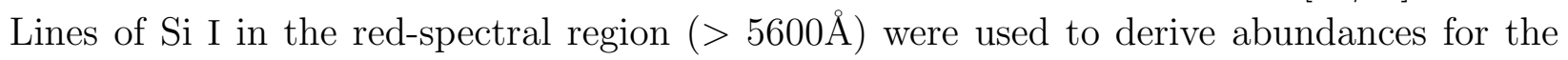
rest of the RHB stars. There are eight stars that we used at least four lines for determining the abundances. For these stars, we derived $<[\mathrm{Si} \mathrm{I} / \mathrm{Fe}]>=+0.42$, which is consistent with the mean of typical $\alpha$-enhancement in metal-poor stars.

In Figure 14, we summarize the Si I abundances found in large-sample studies and the

10 We could not determine a Si abundance for HD 119516 because our spectrum of the $3905 \AA$ line was corrupted by cosmic rays. 
spectral regions that were used to derive the Si I abundances. All investigators agree on the declining trend of $[\mathrm{Si} \mathrm{I} / \mathrm{Fe}]$ with increasing $T_{\text {eff }}$ among cooler metal-poor stars, and we have shown that the abundances reach a (low) plateau in BHB stars. Resolution of this unsatisfactory situation is beyond the scope of this study.

An important check on the Si abundances is provided by our detection of Si II, which has mainly been studied in stars with $T_{\text {eff }}>10,000 \mathrm{~K}$. Only a handful of dwarfs have reported Si II abundances (see Stephens \& Boesgaard 2002), and no prior investigation has been done for RHB stars. In general, Si II lines are very weak for RHB stars, only becoming strong $(\mathrm{EW}>30 \mathrm{~m} \AA)$ in BHB stars. We caution that weak lines and 1-3 Si II lines were used for deriving the Si II abundances.

In Figure 15, we illustrate the mixture of lines that have been used to derive Si II abundances for both RHB and BHB stars. The scatter of [Si II/Fe] is large but the mean abundances agree with the general $\alpha$-enhancement indicated by $\mathrm{Mg}$ and Ca for our HB stars. We find unusually large Si II abundances for CS 22955-174 and CS 22937-072. However, they show normal enhancement in Si I (i.e., +0.3 and +0.5 dex, respectively). Unfortunately, in both cases, only 1-2 Si I or Si II lines were used to derive their abundances, so these abnormally large abundances should be viewed with caution.

\subsection{Light Odd-Z Elements Sodium and Aluminum}

For sodium abundances, we used mainly the Na I resonance D-lines (5889.9 $\AA, 5895.9$ $\AA$ ). Only a few of the cooler RHB stars have detectable, albeit weak, higher excitation Na I lines (the 5682.6 $\AA$, 5688.2 $\AA$ and the $6154.2 \AA, 6160.7 \AA$ doublets). We visually inspected the D-line spectral region to search for ISM contamination of the stellar lines. Any suspected line blending resulted in dropping the D-line measures for a star. The derived $[\mathrm{Na} / \mathrm{Fe}]$ values exhibit a large star-to-star scatter (see Figure 8). We warn the reader that the Na I D-lines are relatively strong in the RHB stars as compared to the BHB stars. Unfortunately, there are only two BHB stars in our samples that have measurable, clean D-lines. Therefore, we could not make direct comparison with the star-to-star scatters in BHB and RHB stars. Nevertheless, the large variations derived here are consistent with those seen in previous field metal-poor star studies (see Pilachowski et al. 1996; Venn et al. 2004 and references therein).

Aluminum is underabundant in RHB stars, $<[\mathrm{Al} / \mathrm{Fe}]>\simeq-0.64$, and overabundant in $\mathrm{BHB}$ stars, $<[\mathrm{Al} / \mathrm{Fe}]>\simeq+0.36$ (see Figure 8 ). There are only two $\mathrm{Al} \mathrm{I}$ lines, the resonance transitions $3944 \AA$ and $3961 \AA$ in the blue spectral region, which we can employ for this study. 
The $3944 \AA$ line can be contaminated by CH transition (Arpigny \& Magain 1983). However, it is not an issue in our very warm BHB stars and it is even undetectable in our metal-poor RHB stars. Additionally, the $3961 \AA$ line can only be a reliable abundance indicator in metalpoor stars, as it is affected by the strong wing of $\mathrm{Ca}$ II $\mathrm{H}$ and $\mathrm{H}_{\epsilon}$ features in higher metallicity stars (Sneden \& Lawler 2008). Higher excitation Al lines in the red spectral region, e.g., the $6696 \AA, 6698 \AA$ pair, generally result in higher [Al/Fe] (see discussion of Francois 1984). The discrepancy of $[\mathrm{Al} / \mathrm{Fe}]$ between the transitions of red and the blue spectral region is currently not completely understood. Unfortunately we could not detect the red Al I lines in our stars.

As noted by others, Na D lines and the Al I red and blue resonance spectral region can be significantly altered from NLTE effects. These corrections are important for warm, metal-poor turnoff stars with $T_{\text {eff }} \gtrsim 6000 \mathrm{~K}$ (Baumueller et al. 1998). The suggested NLTE corrections are -0.5 dex for Na (Baumueller et al. 1998) and +0.65 dex for Al (Baumueller \& Gehren 1997). Since the majority of our RHB stars are below this $T_{\text {eff }}$ we only applied NLTE corrections of suggested values to $\mathrm{Na}$ and $\mathrm{Al}$ abundance ratios of our BHB stars.

\subsection{The iron-peak elements: Scandium through Zinc}

Scandium lines can have substantial hypefine substructure. We synthesized a few Sc II lines with their full substructure, and found that the abundances derived from synthesis do not differ by more than 0.05 dex from those derived by the single-line EW method. Thus, we used the EW method for deriving all final Sc II abundances. A study by Cohen et al. (2004) showed that there are discrepancies of [Sc II/Fe] among different evolutionary groups of metal-poor stars, in which they are generally enhanced in main sequence stars while RGB stars exhibit deficiencies. Our results are more in accord with those of main-sequence stars, $<[\mathrm{Sc} \mathrm{II} / \mathrm{Fe}]>\simeq+0.13$ (see Figure 9).

Our vanadium abundances come exclusively from V II lines, which were detectable in both RHB and BHB stars. We find no trends of $[\mathrm{V} / \mathrm{Fe}]$ with either $[\mathrm{Fe} / \mathrm{H}]$ or $T_{\text {eff }}$.

Chromium abundances derived from Cr I transitions generally yield smaller abundances than those from Cr II lines in metal-poor stars (e.g, Preston et al. 2006a, Sobeck et al. 2007, and references therein). Ideally, we would have preferred to use recent laboratory transition probabilities for both Cr I (Sobeck et al. 2007) and Cr II (Nilsson et al. 2006) for our study. However, there are no Cr II lines studied by Nilsson et al. (2006) that are routinely detectable 
in our spectra. Therefore, we employed the transition probabilites of detectable $\mathrm{Cr}$ I and Cr II lines from Sobeck et al. (2007) and NIST, respectively. The offset between Cr I \& Cr II remains (see Figure 9). The trend of increasing Cr II with decreasing metallicity is due to large line detection/measurement uncertainty; only 1-2 lines were used in relatively metal-poor, RHB stars. This offset is also present in the detailed $\mathrm{Cr}$ transition probability study of Sobeck et al. (2007). Ionization imbalance or non-LTE effect could be the cause.

A trend of increasing $[\mathrm{Cr} \mathrm{I} / \mathrm{Fe}]$ with increasing $T_{\text {eff }}<7000 \mathrm{~K}$ has also been found for RHB stars (see Figure 12). This is first pointed out by Lai et al. (2008) (see their Figure 21). Clearly no such trend is apparent in our BHB stars.

Manganese abundances of field and halo metal-poor dwarf and giant stars have been shown to be substantially underabundant (see, e.g, Sobeck et al. 2006, Lai et al. 2008, and references therein). Our analysis yields $<[\mathrm{Mn} / \mathrm{Fe}]>\simeq-0.35$. The general trend of increasing $[\mathrm{Mn} / \mathrm{Fe}]$ with at higher $[\mathrm{Fe} / \mathrm{H}]$ metallicities in our HB sample is in agreement with those and other previous studies. We refer the reader to review the extensive discussion of Sobeck et al. (2006) regarding the production of $\mathrm{Mn}$.

We derived nickel abundances via spectrum synthesis of the Ni II $4067 \AA$ line and the remaining iron-group elements from EW analysis. The reader should be cautious in interpreting the Co I, Ni II, and Zn I abundances, as they were determined with only 1-2 lines each. There are insufficient data to define an abundance pattern of Ni II at this point. Our $[\mathrm{Ni} 1 / \mathrm{Fe}]$ values are generally near solar for moderately metal-poor stars $([\mathrm{Fe} / \mathrm{H}]>2.0)$. The larger star-to-star scatter for very metal-poor stars $([\mathrm{Fe} / \mathrm{H}]<2.0)$ is probably not real, as only one weak Ni I line was used in our analysis, resulting in uncertain $\mathrm{Ni}$ abundances for individual stars.

Zinc has multiple abundant isotopes $\left({ }^{64,66,67,68} \mathrm{Zn}\right)$, but the isotopic/hyperfine substructure of Zn I lines are not large and the observed features are weak (Timmes et al. 1995). Therefore we treated $\mathrm{Zn}$ I lines as single absorbers. The discussion of $[\mathrm{Zn} / \mathrm{Fe}]$ will be given in $\S 8.1$.

\subsection{The neutron capture elements: Strontium, Yttrium, Zirconium, Barium, Lanthanum and Europium}

We derived the strontium abundances using available Sr II $4077 \AA, 4161 \AA$ and 4215 $\AA$ lines. These lines are particularly hard to analyze in RHB stars because they are strong and/or partially blended. For example, the $4077.8 \AA$ resonance line can be affected by Dy II 4078.0 $\AA$ and possibly La II 4077.3 $\AA$. We illustrate this in Figure16, which shows an example 
of the Sr II 4077 $\AA$ synthesis superimposed on the observed spectrum of an RHB star. The Dy abundance cannot be determined reliably with the spectra. Therefore, the adopted Dy abundance was arbitrarily changed to produce the best fit to the red wing of the observed Sr II line profile.

The star-to-star scatter in $\mathrm{Sr}$ abundances is large (see Figure 101). These variations are intrinsic to the stars, as can be easily seen in the spectra. In Figure 17 we show a few examples. Comparison of stars with similar stellar parameters (i.e., CS 22186-005 and CS 22875-029 in this figure) shows that the large scatter in [Sr/Fe] ratios is real. We also note an offset $(\sim 0.5 \mathrm{dex})$ of $\mathrm{Sr}$ abundance ratios between the RHB and BHB stars, which is not present in Yttrium and Zirconium abundance ratios (see Figure 10 \& 13). This offset may be related to the large Sr II line strength difference between the two HB groups. Additionally, contamination of the lines by other species, which plagues the RHB spectra, is not an issue in the BHB stars.

We performed EW analysis for Yttrium lines. The star-to-star scatter is also large in this element but the analytical uncertainties are smaller for $\mathrm{Y}$ abundances. We compare a Y II line in stars with similar metallicity in Figure18, The comparison shows that stars with similar metallicity possess different [Y II/Fe].

Synthesis were performed for Zr II $4149 \AA, 4161 \AA, 4090 \AA$ and $4317 \AA$ lines, whenever present in the spectra. Generally Zr appears to be overabundant as compared to its neighboring light $n$-capture elements $\mathrm{Sr}$ and Y. We caution that the Zr II lines are generally very weak, and the resulting abundance uncertainties are thus large.

Barium is a much-studied member of the heavier $n$-capture element group. Its lines are affected by both hyperfine substructure and isotopic splitting. A line list with full Ba II substructure is given in McWilliam (1998). We adopted the solar abundance ratio distribution among the ${ }^{134--138} \mathrm{Ba}$ isotopes (Lodders 2003), and synthesized the Ba II lines at $4554 \AA, 5853 \AA, 6141 \AA$, and $6496 \AA$, whenever present in the spectra. We note that the $4554 \AA$ line is always substantially stronger than the other lines, and Ba abundances derived from this line can be severely affected by microturbulence and damping.

The spectral lines of La have significant hyperfine substructure, and those of Eu have both hyperfine substructure and isotopic substructure. There are two natural occurring isotopes, ${ }^{151,153} \mathrm{Eu}$, for which we adopted the solar abundance ratio distribution (Lodders 2003). We employed La II $4086 \AA$ and $4123 \AA$ lines and Eu II 4129 and $4205 \AA$ lines for abundance analysis. In general, Eu and La lines are very weak. None are detectable in BHB stars, and only 1-2 lines are available in RHB stars. 


\section{Evolutionary States}

\section{1. $T_{\text {eff }}-\log g$ Plane}

We investigated the physical properties of our HB samples, by comparing our derived temperatures and gravities using the $\alpha$-enhanced, HB models of Pietrinferni et al. (2006). These models implemented the low T-opacities of Ferguson et al. (2005) and an $\alpha$-enhanced metal distribution that represents typical Galactic halo and bulge stars. The $\alpha$-enhancement treatment is particularly important because the $\alpha$-elements are overabundant in metal-poor stellar atmospheres, and they are major donors of electrons for the for $\mathrm{H}^{-}$continuum opacity. We adopted the HB canonical models of various metallicities with $\eta=0.4$. The models of Pietrinferni et al. were chosen because they provide a fine grid of masses and time steps in contrast to other available HB models.

In order to convert the bolometric luminosities $L / L_{\odot}$ of the models for each mass to $\log g$ values, we adopted Eq. (2) of Preston et al. (2006a),

$$
\log g=\log \left(M / M_{\odot}\right)+4 \log T_{\text {eff }}-\log \left(L / L_{\odot}\right)-10.607
$$

in which the constant was evaluated by using the solar $T_{\text {eff }}$ and $\log g$ values. In Figure 19, we show the spectroscopic $T_{\text {eff }}$ and $\log g$ values of our stars and the field RR Lyraes that are based on spectroscopic $T_{\text {eff }}$ and $\log g$ of Lambert et al. (1996), and, photometric $T_{\text {eff }}$ and Baade-Wesselink log $g$ of Clementini et al. (1995), on the $T_{\text {eff }}-\log g$ plane. Both their data and our samples exhibit similar gravity scatter at fixed temperature.

To estimate the uncertainties associated with the Pietrinferni et al. (2006) HB models, we compare their luminosities (as translated into log $g$ ) for a given mass with Lee \& Demarque (1990)'s HB model (i.e., $[\mathrm{Fe} / \mathrm{H}]=-2.26, Z=0.0001, Y=0.23) 11$ The comparison is summarized in Table 12. The difference in $\log g$ in the two studies is $\lesssim 0.1$ dex, much smaller than the uncertainties in our spectroscopic $\log g$ values. Therefore, model choice is not an issue in contributing significant error on the mass derivation.

\subsection{Derivation of HB Masses}

Our mass estimation uses HB evolutionary tracks in the $T_{\text {eff }}-\log g$ plane. As discussed in $§ 5.1$, spectroscopic $\log g$ values are generally lower than the photometric ones, which

\footnotetext{
11 Dorman et al. (1993) also published HB models with similar parameters, but their time steps are too large to be useful in this exercise.
} 
would result in deriving more of low mass HB stars. Therefore, a correction of the spectroscopic gravities is necessary and adopting the photometric gravities is more appropriated to represent the physical gravities.

Preston et al. (2006a) derived an empirical relation for computing photometric gravities $\left(\log g_{\text {phot }}\right)$ by using their spectroscopic gravities $\left(\log g_{\text {spec }}\right)$ in conjunction with the existing $\log g_{\mathrm{phot}}$ of M 15. We adopted this relation,

$$
\log g_{\text {phot }}=\log g_{\text {spec }}+28.802-7.655 \log T_{\text {eff }, \text { spec }}
$$

to obtain the $\log g_{\text {phot }}$ for all our RHB stars. While there are published log $g_{\text {phot }}$ data for BHB stars in other GCs (Behr 2003a), there are no useful log $g_{\text {spec }}$ values for comparison (Behr 2003a suggested that their measurements are too uncertain to provide any useful information on this issue). Additionally, Preston et al. showed that the corrections to log $g_{\text {spec }}$ decline with increasing $T_{\text {eff }}$ and essentially disappear at the red edge of RR Lyr IS (see their Figure 17). This can be understood by noting that the continuous opacity of a hotter star is dominated by $\mathrm{H}^{-}$, and the dominant electron donor is hydrogen itself rather than the metals. The electron density rises sharply with increasing $T_{\text {eff }}$ among RHB stars. Examination of atmosphere models for the M15 RHBs (from Preston et al.) suggests that in the line-forming regions, the electron pressure increases by a factor of more than 30 from the coolest $\left(T_{\text {eff }}\right.$ $=5000 \mathrm{~K})$ to the warmest $\left(T_{\text {eff }}=6250 \mathrm{~K}\right)$ stars. This higher electron pressure helps to enforce LTE in the ionization equilibria in warmer HB stars. Thus, we assume the spectroscopic $\log g$ for our BHB stars is correct and no correction is applied. Future spectroscopic investigation of $\log g$ for BHB stars in GCs would be welcome.

After calculating RHB log $g_{\text {phot }}$ values, we estimated the masses of individual HB star by employing an interpolation scheme. To account for different metallicities of our program stars, we first chose two models that closely match a star's $[\mathrm{Fe} / \mathrm{H}]$ and superimposed them on the $T_{\text {eff- }} \log g$ plane along with the $T_{\text {eff,spec }}$ and $\log g_{\text {phot }}$. Then, calculating the linear interpolation between these two metallicities and masses:

$$
M_{\text {star }}=M_{1}+\frac{\left(M_{2}-M_{1}\right)}{\left([\mathrm{Fe} / \mathrm{H}]_{2}-[\mathrm{Fe} / \mathrm{H}]_{1}\right)} \times\left([\mathrm{Fe} / \mathrm{H}]_{\mathrm{star}}-[\mathrm{Fe} / \mathrm{H}]_{1}\right)
$$

where $M_{1}, M_{2}$ are estimated masses from the two models, and $[\mathrm{Fe} / \mathrm{H}]_{1},[\mathrm{Fe} / \mathrm{H}]_{2}$ are the two models' iron abundances. For stars positioned outside the model mass range $\left(0.503 M_{\odot} \leq\right.$ $\left.M \leq 0.80 M_{\odot}\right)$, we chose the mass that is within the $\log g$ and $T_{\text {eff }}$ errors of the star on $T_{\text {eff }}-\log g$ plane. If there is no mass track lies within the errors, we constrain the upper mass limit to be $0.8 M_{\odot}$, the approximate turnoff mass of a old metal-poor main-sequence star. In Figure 20, we show an example of a set of HB stars superimposed on the HB tracks $([\mathrm{M} / \mathrm{H}]=-1.79$ and -2.27$)$ that were used to derive their masses. We summarize the derived 
masses as a histogram in Figure 21 and parameters used to derive the masses is listed in Table 13 .

The inferred mass distributions have means at $0.59 M_{\odot}$ and $0.56 M_{\odot}$ for $\mathrm{RHB}$ and $\mathrm{BHB}$ stars, respectively (see Figure 21). If we exclude those RHB stars that have masses set to the upper limit $\left(M>0.8 M_{\odot}\right)$, the mean masses for RHB and BHB stars are both $0.56 M_{\odot}$, and the median masses are $0.54 M_{\odot}$ and $0.56 M_{\odot}$.

This estimated mean mass is smaller than the HB masses found in some GCs, e.g. M3, for which Valcarce \& Catelan (2008) derived mean masses of $0.633 M_{\odot}$ and $0.650 M_{\odot}$ for RHB and BHB stars, respectively. We also do not find a bimodal or multi-modal HB mass distribution that appears to exist in many GC's (see Valcarce \& Catelan; Catelan 2004). Several reasons could contribute to these differences. (1) GC's are mostly monometallic, in contrast to the large metallicity range of our FHB stars. We have needed to multiple evolutionary tracks that correspond most closely to the individual metallicities of our FHB stars (refer back to the interpolation method as described above). (2) Our sample sizes of RHB and BHB stars are too small to clearly indicate statistically significant mass distributions. (3) We have used an empirical correction to spectroscopically-determined log $g$ values, which directly impacts the derived masses. (3) Our samples consist more of RHB than BHB stars, where the majority agglomerate near the low mass end, resulting in more low mass HB estimates. (4) Finally, Valcarce \& Catelan cautioned about over-interpretation of masses derived from the GC CMD method, because they are biased against stars in later evolutionary states. Thus, it is not clear that our mean masses are substantially different than those reported for M3.

Additionally, other GC HB mass study have reported mean mass in reasonable agreement with ours. For example, de Boer et al. (1993) obtained $<M_{\mathrm{HB}}>=0.5 M_{\odot}$ for NGC 6397. Masses of nearby HB stars derived via Hipparcos parallaxes have slightly smaller mean masses, $\left\langle M_{\mathrm{HB}}>=0.38 M_{\odot}\right.$, than ours (de Boer et al. 1997). Finally, the evolutionary

and structural models of Sweigart (1987) suggest a wide range of individual HB masses (0.2 - $\left.1.2 \mathrm{M}_{\odot}\right)$. We conclude that our derived mean masses for the field HB stars are reasonable.

\subsection{Blue and Red Edges of the RR Lyrae Instability Strip: $[\mathbf{F e} / \mathbf{H}]>-2.5$}

Locations of the blue and red edges (BE and RE) of the RR Lyr IS provide powerful constraints on stellar pulsation theory. They can be determined directly by examining the color-magnitude diagram of GCs that are well populated with RR Lyrs. Unfortunately, this requirement eliminates most clusters. 
Additionally, accurate cluster reddenings must be known to transformation from colors to $T_{\text {eff }}$ values. Determining the blue and red edges from bright field RR Lyr stars via spectroscopic method can avoid these complications. For the metallicity regime $[\mathrm{Fe} / \mathrm{H}]<$ -2.0 , Preston et al. (2006a) estimated the fundamental red edge from the $T_{\text {eff }}$ distributions of field RHB stars and GC RR Lyrs. Since HB colors are affected by metallicity, shifting slightly blueward with decreasing [Fe/H] (e.g., see Figure 1 of Sandage 1990), we repeated the exercise with our sample. We considered only those stars with $[\mathrm{Fe} / \mathrm{H}]>-2.5$, and compared the $T_{\text {eff }}$ distributions of our field RHB and BHB with the distribution for field RR Lyr stars.

In Figure 22, the top and bottom panels show the distributions of spectroscopic and photometric $T_{\text {eff' }}$ 's of BHB and RHB stars with $[\mathrm{Fe} / \mathrm{H}]>-2.5$, respectively. The data for field RR Lyr stars (fundamental mode RRab and first overtone RRc variables) in both middle panels are extracted from Lambert et al. (1996) and Clementini et al. (1995). It shows the RR Lyr distribution drops at $T_{\text {eff }}=5900 \mathrm{~K}$ and $7000 \mathrm{~K}$. Both photometric and spectroscopic $T_{\text {eff }}$ RHB distributions decline at $T_{\text {eff }}>5700 \mathrm{~K}$ and overlap with the RR Lyr distributions (bottom panels). We suggest that the weak overlap region, $\simeq 5900 \mathrm{~K}$, is the red edge of field $\mathrm{HB}$ with $[\mathrm{Fe} / \mathrm{H}]>-2.5$. The $T_{\text {eff }}$ 's of our BHB sample have no overlap with those of the RR Lyr stars. This is expected since RRc type variables, which are bluer than the RRab type variables, are generally used for determining the BE, and there are only two RRc type variables from Lambert et al. (1996) being included in the histogram (middle panels). Assuming the RRc type variables defined the blue edge in this case, we approximated it to be $7400 \mathrm{~K}$.

While field HB stars can be used for deriving red and blue edges, we warn that the method is not very robust. The lack of large BHB samples and uncertainties in $T_{\text {eff }}$ values of field RRc stars are limiting factors on our blue edge estimates. The overlapping distributions of field RHB and RRab stars also limit the red edge accuracy. Perhaps semi-empirical work (i.e., simulations to map the observed distributions) would provide a better constraints on the red and blue edges of FHB stars. Before then, deriving $T_{\text {eff }}$ 's for a large sample of field BHB and RRc will be needed.

\section{Discussion}

In this paper we have explored the chemical compositions of non-variable RHB and BHB field stars. Here we will compare our results with abundances in other evolutionary groups of halo field stars, and discuss some of the possible nucleosynthetic implications. The comparisons of our $[\mathrm{X} / \mathrm{Fe}]$ values with those of field stars are presented in Figure 23-25, where neutral and ionized species abundances of several elements have been averaged. We 
did not combine Cr I \& Cr II abundances, since their distributions conspicuously diverge at lower metallicities (as discussed in $\S 6.4$ ). Data for field stars were mainly taken from the compilation of Venn et al. (2004). For those [X/Fe] that are not listed in Venn et al. (2004), we assembled the comparison samples from several references, which we summarize in Table 14.

\subsection{Light and Iron-peak Elements}

Enrichment of $\alpha$-elements in metal-poor stars has been known for decades. The explanation for this behavior presumes predominance of nucleosynthetic contributions from shortlived massive stars that died in core-collapse type II supernovae (SNe II) in early Galactic times. The resulting explosions contributed large amounts of light $\alpha$-elements (e.g., O, Ne, $\mathrm{Mg}$ and $\mathrm{Si}$ ), smaller amounts of heavier $\alpha$-elements (e.g., $\mathrm{Ca}$ and $\mathrm{Ti}$ ) and small amounts of Fe-peak elements to the ISM (Woosley \& Weaver 1995). Longer-lived, lower-mass stars began to contribute their ejecta by adding more Fe-peak elements through Type Ia supernovae (SNe Ia) from lower-mass progenitors which exploded in thermonuclear runaway processes at later times. When SNe Ia became significant polluters of the ISM, a lowering of the $[\alpha / \mathrm{Fe}]$ values (at higher metallicities) occurred.

In general our $\mathrm{HB} \alpha$-element abundances agree with those of other halo star populations. We illustrate this in Figure 23, where $[\mathrm{Mg} \mathrm{I} / \mathrm{Fe}]$ and $[\mathrm{Ti} \mathrm{I} / \mathrm{Fe}]$ of our RHB and BHB are in close accord with other field stars. The $<[\mathrm{Si} \mathrm{I}+\mathrm{II} / \mathrm{Fe}]>$ and $<[\mathrm{Ca} \mathrm{I}+\mathrm{II}]>$ of RHB stars follow the general field star trend but these ratios tend to be lower for BHB stars in the same metallicity range (i.e., $\sim 0.35$ dex lower). The offset of mean Ca abundances is mainly due to the lower $[\mathrm{Ca} \mathrm{I} / \mathrm{Fe}]$ of BHB stars (see description in $\S 6.1$ ). Similar lines were used in both BHB and RHB stars, as such, line selection is probably not the cause of the offset. As for $<[\mathrm{Si} \mathrm{I}+\mathrm{II} / \mathrm{Fe}]>$, the star-to-star scatter is large and the offset between RHB and BHB stars is dominated by the RHB star [Si I/Fe] dependence on $T_{\text {eff }}$ (see $\S 6.2$ ).

Our BHB and RHB sodium abundance pattern looks quite different than in other field stars. However, little weight should be attached to our results because they have large uncertainties. We must rely solely on the Na D lines, and they are very strong in RHB stars. Aluminum is produced in massive stars, similarly to magnesium, but significantly deficient with respect to iron in metal-poor stars. The production of $\mathrm{Al}$ rises as it reaches the diskto-halo transition at higher metallicity, i.e., $[\mathrm{Fe} / \mathrm{H}] \gtrsim 1.5$ (e.g., Timmes et al. 1995). Our abundances confirm this, with the caution that our derived trend with metallicity depends solely on RHB stars at low $[\mathrm{Fe} / \mathrm{H}]$ and all BHB stars at high $[\mathrm{Fe} / \mathrm{H}]$. 
Iron-peak elements (with the exception of $\mathrm{Ti}$, discussed above) are believed to be largely produced during Type Ia and Type II SNe explosion events. In our metallicity regime the iron-peak abundances of main-sequence and RGB stars generally have their solar values, with the exception of $\mathrm{Mn}$ and $\mathrm{Cu}$. The derived Fe-peak abundance ratios (i.e., Sc II, Cr I, and V II) of our RHB and BHB stars are also in agreement with those found in field dwarfs and giants (see Figure 24). Most of them are expected to be constant in all metallicity regimes. Manganese and Zinc are the exceptions. In common with previous studies, $[\mathrm{Mn} / \mathrm{Fe}]$ ratios of our HB stars increase as metallicity increases, but the slope of this relation may be larger in our sample. We do not have a clear physical explanation to this, and caution that, (a) the trend is based on relatively few points, and (b) $[\mathrm{Mn} / \mathrm{Fe}]$ is quite sensitive to stellar parameter choices (refer to Table 10 \& 11). Again, we refer the reader to Sobeck et al. (2006) for the production of $\mathrm{Mn}$.

For nickel abundances we must rely on $\mathrm{Ni}$ I lines for RHB stars and $\mathrm{Ni}$ II lines for BHB stars. The low Ni II abundances of BHB stars should not be given large weight, as they are solely derived from one line. The very large [Ni I/Fe] values of several RHB stars, substantially at variance with the general trend of field stars, are most likely due to the lack of many detectable lines. The RHB stars with more than four lines contributing to their $\mathrm{Ni}$ abundance have ratios in good agreement with the field stars.

We find $[\mathrm{Zn} / \mathrm{Fe}] \simeq 0.0$ throughout the metallicity regime of $[\mathrm{Fe} / \mathrm{H}]>-2.0$, which is consistent with the study of Sneden et al. (1991). Recent work by Cavrel et al. (2004) shows increasing $[\mathrm{Zn} / \mathrm{Fe}]$ at decreasing metallicities. Such a trend could indicate an $\alpha$-rich freezeout process contribution to Fe-group element production at low metallicities. Our Zn abundance at low metallicity range, i.e., $[\mathrm{Fe} / \mathrm{H}]<-2.0$, perhaps consistent with this recent finding, but our data points are too sparse for firm conclusions on this point. Unfortunately, the comparison can only be made for RHB stars since the Zn I lines in BHB stars are too weak to be detected.

\subsection{Neutron-Capture Elements}

Elements heavier than the iron-peak $(\mathrm{Z}>30)$ cannot be efficiently synthesized by charged-particle fusion because of Coulomb repulsion and the endothermic nature of such reactions. They are produced in the late stages of stellar evolution via neutron-capture events, namely the $s$ - and $r$-processes (see review by Sneden et al. 2008). The $s$-process

occurs quiescently in the He-fusion zones of low or intermediate mass AGB stars, while the $r$-process is believed to occur explosively in neutron rich sites, e.g., Type II SNe or merging events of two neutron stars (Rosswog et al. 1999). 
We have abundances for six $n$-capture elements in HB stars. Strontium, Yttrium and Zirconium are relatively light $n$-capture elements. In the solar system, they are attributed mostly to the "main" $s$-process (Arlandini et al. 1999). Barium and Lanthanum are heavier $n$-capture elements also primarily $s$-process elements in solar-system material. Europium is our sole representative of solar-system $r$-process elements.

Our HB $n$-capture abundance ratios are generally in accord with field stars studies (see Figure 25). The offset of $[\mathrm{Sr} / \mathrm{Fe}]$ between RHB and BHB stars are discussed in $\S 6.5$. Unfortunately, we do not have $[\mathrm{Sr} / \mathrm{Fe}]$ for field stars with $[\mathrm{Fe} / \mathrm{H}]>-2.0$ for comparison. The resonance lines of Sr II are very strong for moderately metal-poor cooler stars and thus Strontium is not well represented in previous field-star surveys in this metallicity regime. We conclude that $<[\mathrm{Sr} / \mathrm{Fe}]>\sim 0$ for $[\mathrm{Fe} / \mathrm{H}]>-2.0$.

Increasing star-to-star scatter with decreasing metallicity is apparent in the heavier $n$ capture elements $\mathrm{Ba}$, La, and $\mathrm{Eu}$, in accord with trends seen in other field star samples. A sharp downward trend of $[\mathrm{Ba} \mathrm{II} / \mathrm{Fe}]$ with decreasing metallicity becomes apparent for $[\mathrm{Fe} / \mathrm{H}]<-2.0$. This pattern is present in field stars studies as well. The $[\mathrm{La} / \mathrm{Fe}]$ should roughly correlate with $[\mathrm{Ba} / \mathrm{Fe}]$. Unfortunately, we cannot easily detect La II lines in HB stars below $[\mathrm{Fe} / \mathrm{H}] \simeq-2.5$, where the drop in Ba abundance becomes apparent. The simplest explanation for the rise of $[\mathrm{Ba} / \mathrm{Fe}]$ at $[\mathrm{Fe} / \mathrm{H}]>-2.0$ is that the $r$-process dominates $\mathrm{Ba}$ production at lowest metallicities while the $s$-process plays a more important role at higher metallicities (Busso et al. 1999).

The initial examination of our derived Europium abundances yielded six RHB stars with $[\mathrm{Eu} / \mathrm{Fe}]>0.5$, well above the mean trend. However, high $[\mathrm{Eu} / \mathrm{Fe}]$ has also been found in some field stars (as shown in Figure 25). For example, $n$-capture rich star CS 22892-052 has $[\mathrm{Eu} / \mathrm{Fe}]=+1.64$ (Sneden et al. 2003) and CS 31082-001 has $[\mathrm{Eu} / \mathrm{Fe}]=+1.63$ (Hill et al. 2002). The other $n$-capture elements of three of the Eu-rich RHB stars in our samples, i.e., CS 22875-029, CS 22886-043 and BD $+17^{\circ} 3248$ are also high, implying that these three are truly $n$-capture rich stars. The overall $n$-capture abundance distributions for the other three RHB stars with Eu excesses are less certain. These six RHB stars deserve followup spectroscopic investigation of the $n$-capture elements.

\subsection{Heavier vs Lighter Neutron-Capture Elements}

Abundances of light $n$-capture elements Sr, Y, and Zr appear to be highly correlated with each other, and clearly they share a common nucleosynthetic origin (e.g., McWilliam et al. 1995; Francois et al. 2007; Aoki et al. 2005). In Figure 26, we compare the mean Sr-Y-Zr 
abundances the heavier element Ba for our HB stars, adding in the data of Francois et al. (2007). Only stars with detections of all of these elements are included in this plot. The comparison shows a tight correlation (i.e., increasing overabundant as decreasing Barium abundances), which suggests the correlation exists regardless of metallicity regime and evolutionary state.

To examine the contributions of the $r$ and $s$-process ratios of metal-poor stars, abundances of Y, Ba, La and Eu are generally used. As discussed above, Y, Ba and La can be formed via $r$ and $s$-processes, while $\mathrm{Eu}$ is largely formed via the $r$-process. In Figure 27 , we plotted the $[\mathrm{La} / \mathrm{Eu}],[\mathrm{Ba} / \mathrm{Eu}]$ and $[\mathrm{Y} / \mathrm{Eu}]$ vs $[\mathrm{Fe} / \mathrm{H}]$ of our HB samples along with those of Venn et al. (2004), Simmerer et al. (2004) and Woolf et al. (1995), and compare them with estimated pure $r$-process solar system abundances (Arlandini et al. 1999; Sneden et al. 2008).

The top panel shows the $[\mathrm{La} / \mathrm{Eu}]$ distribution, which the rise of $[\mathrm{La} / \mathrm{Eu}]$ as metallicity increases progresses slower than $[\mathrm{Ba} / \mathrm{Eu}]$ and $[\mathrm{Y} / \mathrm{Eu}]$. The comparison between $[\mathrm{La} / \mathrm{Eu}]$ and middle panel of $[\mathrm{Ba} / \mathrm{Eu}]$ demonstrates that the larger scatter of $[\mathrm{Ba} / \mathrm{Eu}]$ is due to the Barium not Europium abundances. The middle and bottom panels of $[\mathrm{Ba} / \mathrm{Eu}]$ and $[\mathrm{Y} / \mathrm{Eu}]$ show large scatter in very metal-poor stars regime, which suggests an inhomogeneous mixing in early Galactic time. We also find a slow increase of $[\mathrm{Ba} / \mathrm{Eu}]$ and $[\mathrm{Y} / \mathrm{Eu}]$ as the metallicity increases. The rise is further evidence of the increasing contribution of the $s$ process as metallicity increases (with time in the Galaxy). The slope of [Ba/Eu] for our HB stars is steeper than the field stars but the overall trend is indistinguishable from the large scatter. Also, the $[\mathrm{Y} / \mathrm{Eu}]$ abundances are above the estimated pure $r$-process solar-system abundances, which again suggests that the $s$-process (from AGB stars) play a significant role in Yttrium production.

\subsection{CS 22186-005}

The RHB star CS 22186-005 has an extremely low Sr abundance, i.e., [Sr II/Fe] = -1.03 (see Figures $17 \&$ 25). As expected, there is no detection of the weaker Zr II and Y II in this star. However, we detected Barium, with an abundance ratio of $[\mathrm{Ba} \mathrm{II} / \mathrm{Fe}]=-0.58$. Its Barium abundance follows the general declining trend of metal-poor stars that has metallicity below -2.0 (see Figure [25). The resulting abundance ratio, $[\mathrm{Ba} / \mathrm{Sr}]=+0.45$, is somewhat surprising because in most $n$-capture metal-poor cases, the heavier $n$-capture elements are underabundant with respect to lighter ones (as summarized in see Figure 7 of Sneden et al. 2008). Other heavier $n$-capture elements (i.e., Eu and La) were not detectable with our spectra of CS 22186-005, This star does not appear to have obvious abundance anomalies 
among the lighter elements.

In Figure 28, we extend Sneden et al's Figure 7 by adding in $\mathrm{Sr}$ and Ba abundances of our RHB and BHB stars. It is clear that CS 22186-005 is not the only metal-poor star that exhibits unusually large $[\mathrm{Ba} / \mathrm{Sr}]$ ratios at low $[\mathrm{Ba} / \mathrm{Fe}]$. Such stars have mainly been found among the very metal-poor giant sample of François et al. (2007). Clearly these stars provide further evidence that $n$-capture synthesis events cannot easily be characterized by single nucleosynthesis processes. Followup observations at higher $\mathrm{S} / \mathrm{N}$ and resolution of this type of star should be undertaken.

\section{Conclusions}

We present the first large-scample detailed chemical composition study of non-variable field RHB and BHB stars. The high resolution spectra for our work were obtained with the $2.7 \mathrm{~m}$ telescope at the McDonald Observatory. The sample was selected from the survey of Behr (2003b). Additional RHB spectra from Preston et al. (2006a) were also added to the analysis. We derived the model stellar atmospheric parameters, $T_{\text {eff }}, \log g,[\mathrm{Fe} / \mathrm{H}]$, and $v_{\mathrm{t}}$ for all program stars based on spectroscopic constraints. Of some interest is that the microturbulence of RHB stars increase with increasing $T_{\text {eff }}$, in agreement with Preston et al. (2006a), while microturbulence appears to decline with increasing $T_{\text {eff }}$ in BHB stars. More data on BHB stars to solidify this conclusion would be welcome.

Employing these stellar parameters, we derived relative abundance ratios, $[\mathrm{X} / \mathrm{Fe}]$, of the $\alpha$-elements, Fe-peak elements and $n$-capture elements for these stars. The abundance ratios vs metallicity of our $\mathrm{RHB}$ and $\mathrm{BHB}$ stars are generally in accord with other field star studies. In particular, the $\alpha$-elements are overabundant, [Al I/Fe] (RHB stars only) and $[\mathrm{Mn} \mathrm{I} / \mathrm{Fe}]$ are underabundant for metal-poor stars. Large star-to-star scatter is present in $[n$-capture/Fe] abundance ratios.

Finally we investigated the physical properties of our RHB and BHB stars by locating them in the $T_{\text {eff }}-\log g$ plane, and comparing them to HB evolutionary tracks of Pietrinferni et al. (2006), in order to estimate individual stellar masses. The mass distribution suggests that the majority of our stars have $M \sim 0.56 M_{\odot}$. By comparing the $T_{\text {eff }}$ distribution of our field RHB and BHB stars with the field RR Lyraes of Lambert et al. (1996) and Clementini et al. (1995), we estimated the temperatures of red and blue edges of the RR Lyr IS for stars with $[\mathrm{Fe} / \mathrm{H}]>-2.5$. We derived $5900 \mathrm{~K}$ and $7400 \mathrm{~K}$, respectively for these edges.

The general consistency of $\mathrm{HB}$ abundance ratios with those of other dwarf and giant 
halo star samples justifies that HB stars can be used routinely in the future for Galactic sturcture-metallicity studies (such as investigations of stellar streams). More importantly, this work provides a starting point for our future study on chemical compositions of RR Lyrs (For et al., in prep). Determinations of abundances of these stars throughout their pulsational cycles will be examined in detail with the same methods as have been employed in this paper.

B.-Q. For acknowledges the invaluable assistance from the mountain support staff at the McDonald observatory, and travel assistance from a SigmaXi grant-in-aid. We are grateful to Bradford Behr and George Preston for helpful discussions and advice on their earlier studies. This research was supported by U.S. National Science Foundation grants AST-0607708 and AST-0908978.

\section{REFERENCES}

Adelman, S. J., \& Hill, G. 1987, MNRAS, 226, 581

Adelman, S. J., \& Philip, A. G. D. 1990, MNRAS, 247, 132

Allende Prieto, C. 2001, ArXiv Astrophysics e-prints

Allende Prieto, C., García López, R. J., Lambert, D. L., \& Gustafsson, B. 1999, ApJ, 527, 879

Alonso, A., Arribas, S., \& Martinez-Roger, C. 1994, A\&A, 282, 684

Alonso, A., Arribas, S., \& Martínez-Roger, C. 1999, A\&AS, 140, 261

Altmann, M. \& de Boer, K. S. 2000, A\&A, 353, 135

Anders, E., \& Grevesse, N. 1989, Geochim. Cosmochim. Acta, 53, 197

Aoki, W., et al. 2005, ApJ, 632, 611

Arlandini, C., Käppeler, F., Wisshak, K., Gallino, R., Lugaro, M., Busso, M., \& Straniero, O. 1999, ApJ, 525, 886

Arpigny, C., \& Magain, P. 1983, A\&A, 127, L7

Barklem, P. S., \& O’Mara, B. J. 1998, MNRAS, 300, 863

Barklem, P. S., et al. 2005, A\&A, 439, 129 
Baumueller, D., Butler, K., \& Gehren, T. 1998, A\&A, 338, 637

Baumueller, D., \& Gehren, T. 1997, A\&A, 325, 1088

Beers, T. C., Preston, G. W., \& Shectman, S. A. 1992, AJ, 103, 1987

Behr, B. B. 2003a, ApJS, 149, 67

-. 2003b, ApJS, 149, 101

Blackwell, D. E., \& Shallis, M. J. 1977, MNRAS, 180, 177

Burstein, D., \& Heiles, C. 1982, AJ, 87, 1165

Busso, M., Gallino, R., \& Wasserburg, G. J. 1999, ARA\&A, 37, 239

Cassisi, S., Castellani, M., Caputo, F., \& Castellani, V. 2004, A\&A, 426, 641

Castelli, F., Gratton, R. G., \& Kurucz, R. L. 1997, A\&A, 318, 841

Catelan, M. 2004, ApJ, 600, 409

Cayrel, R., et al. 2004, A\&A, 416, 1117

Clementini, G., Carretta, E., Gratton, R., Merighi, R., Mould, J. R., \& McCarthy, J. K. 1995, AJ, 110, 2319

Cohen, J. G., et al. 2004, ApJ, 612, 1107

de Boer, K. S., Geffert, M., Tucholke, H., \& Schmidt, J. H. K. 1997, in ESA Special Publication, Vol. 402, Hipparcos - Venice '97, ed. R. M. Bonnet, E. Høg, P. L. Bernacca, L. Emiliani, A. Blaauw, C. Turon, J. Kovalevsky, L. Lindegren, H. Hassan, M. Bouffard, B. Strim, D. Heger, M. A. C. Perryman, \& L. Woltjer, 331-334

de Boer, K. S., Schmidt, J. H., \& Heber, U. 1993, in Astronomische Gesellschaft Abstract Series, Vol. 9, Astronomische Gesellschaft Abstract Series, ed. G. Klare, 173-+

Demarque, P., Zinn, R., Lee, Y., \& Yi, S. 2000, AJ, 119, 1398

Dorman, B., Rood, R. T., \& O’Connell, R. W. 1993, ApJ, 419, 596

Ferguson, J. W., Alexander, D. R., Allard, F., Barman, T., Bodnarik, J. G., Hauschildt, P. H., Heffner-Wong, A., \& Tamanai, A. 2005, ApJ, 623, 585

Fitzpatrick, M. J., \& Sneden, C. 1987, in Bulletin of the American Astronomical Society, Vol. 19, Bulletin of the American Astronomical Society, 1129-+ 
François, P., et al. 2007, A\&A, 476, 935

Francois, P. 1984, Academie des Science Paris Comptes Rendus Serie B Sciences Physiques, 299, 195

Fulbright, J. P. 2000, AJ, 120, 1841

Fusi Pecci, F., Ferraro, F. R., Bellazzini, M., Djorgovski, S., Piotto, G., \& Buonanno, R. 1993, AJ, 105, 1145

Gratton, R. G., et al. 2007, A\&A, 464, 953

Heber, U. 2009, ARA\&A, 47, 211

Hill, V., et al. 2002, A\&A, 387

Hoyle, F., \& Schwarzschild, M. 1955, ApJS, 2, 1

Hubrig, S., Castelli, F., de Silva, G., González, J. F., Momany, Y., Netopil, M., \& Moehler, S. 2009, A\&A, 499, 865

Johnson, C. I., Kraft, R. P., Pilachowski, C. A., Sneden, C., Ivans, I. I., \& Benman, G. 2005, PASP, 117, 1308

Khalack, V. R., Leblanc, F., Behr, B. B., Wade, G. A., \& Bohlender, D. 2008, A\&A, 477, 641

Khalack, V. R., Leblanc, F., Bohlender, D., Wade, G. A., \& Behr, B. B. 2007, A\&A, 466, 667

Lai, D. K., Bolte, M., Johnson, J. A., Lucatello, S., Heger, A., \& Woosley, S. E. 2008, ApJ, 681,1524

Lambert, D. L., Heath, J. E., Lemke, M., \& Drake, J. 1996, ApJS, 103, 183

Lee, Y., \& Demarque, P. 1990, ApJS, 73, 709

Lee, Y., Demarque, P., \& Zinn, R. 1994, ApJ, 423, 248

Lodders, K. 2003, ApJ, 591, 1220

McWilliam, A. 1998, AJ, 115, 1640

McWilliam, A., Preston, G. W., Sneden, C., \& Searle, L. 1995, AJ, 109, 2757 
Meléndez, J., Shchukina, N. G., \& Vasiljeva, I. E. a nd Ramírez, I. 2006, ApJ, 642, 1082

Möhler, S. 2004, in IAU Symposium, Vol. 224, The A-Star Puzzle, ed. J. Zverko, J. Ziznovsky, S. J. Adelman, \& W. W. Weiss, 395-402

Nilsson, H., Ljung, G., Lundberg, H., \& Nielsen, K. E. 2006, A\&A, 445, 1165

Nissen, P. E., Akerman, C., Asplund, M., Fabbian, D., Kerber, F., Kaufl, H. U., \& Pettini, M. 2007, A\&A, 469, 319

Perryman, M. A. C., et al. 1997, A\&A, 323, L49

Peterson, R. C., Rood, R. T., \& Crocker, D. A. 1995, ApJ, 453, 214

Pietrinferni, A., Cassisi, S., Salaris, M., \& Castelli, F. 2006, ApJ, 642, 797

Pilachowski, C. A., Sneden, C., \& Kraft, R. P. 1996, AJ, 111, 1689

Preston, G. W., \& Sneden, C. 2000, AJ, 120, 1014

Preston, G. W., Sneden, C., Thompson, I. B., Shectman, S. A., \& Burley, G. S. 2006a, AJ, 132,85

Preston, G. W., Thompson, I. B., Sneden, C., Stachowski, G., \& Shectman, S. A. 2006b, AJ, 132, 1714

Ramírez, I., \& Meléndez, J. 2005, ApJ, 626, 465

Reddy, B. E., Tomkin, J., Lambert, D. L., \& Allende Prieto, C. 2003, MNRAS, 340, 304

Rood, R. T., \& Seitzer, P. O. 1981, in IAU Colloq. 68: Astrophysical Parameters for Globular Clusters, ed. A. G. D. Philip \& D. S. Hayes, 369-+

Rosenberg, A., Aparicio, A., Saviane, I., \& Piotto, G. 2000, A\&AS, 145, 451

Rosswog, S., Liebendörfer, M., Thielemann, F., Davies, M. B., Benz, W., \& Piran, T. 1999, A\&A, 341, 499

Sandage, A. 1990, ApJ, 350, 603

Sandage, A., \& Wallerstein, G. 1960, ApJ, 131, 598

Schlegel, D. J., Finkbeiner, D. P., \& Davis, M. 1998, ApJ, 500, 525

Searle, L., \& Zinn, R. 1978, ApJ, 225, 357 
Shi, J. R., Gehren, T., Mashonkina, L., \& Zhao, G. 2009, A\&A, 503, 533

Simmerer, J., Sneden, C., Cowan, J. J., Collier, J., Woolf, V. M., \& Lawler, J. E. 2004, ApJ, 617,1091

Skrutskie, M. F., et al. 2006, AJ, 131, 1163

Sneden, C., Cowan, J. J., \& Gallino, R. 2008, ARA\&A, 46, 241

Sneden, C., Gratton, R. G., \& Crocker, D. A. 1991, A\&A, 246, 354

Sneden, C., \& Lawler, J. E. 2008, in American Institute of Physics Conference Series, Vol. 990, First Stars III, ed. B. W. O’Shea \& A. Heger, 90-103

Sneden, C., et al. 2003, ApJ, 591, 936

Sneden, C. A. 1973, PhD thesis, THE UNIVERSITY OF TEXAS AT AUSTIN.

Sobeck, J. S., Ivans, I. I., Simmerer, J. A., Sneden, C., Hoeflich, P., Fulbright, J. P., \& Kraft, R. P. 2006, AJ, 131, 2949

Sobeck, J. S., Lawler, J. E., \& Sneden, C. 2007, ApJ, 667, 1267

Stephens, A., \& Boesgaard, A. M. 2002, AJ, 123, 1647

Sweigart, A. V. 1987, ApJS, 65, 95

-. 1997, ApJ, 474, L23+

Thévenin, F., \& Idiart, T. P. 1999, ApJ, 521, 753

Timmes, F. X., Woosley, S. E., \& Weaver, T. A. 1995, ApJS, 98, 617

Valcarce, A. A. R., \& Catelan, M. 2008, A\&A, 487, 185

Venn, K. A., Irwin, M., Shetrone, M. D., Tout, C. A., Hill, V., \& Tolstoy, E. 2004, AJ, 128, 1177

Wallerstein, G. qnd Greenstein, J. L., Parker, R., Helfer, H. L., \& Aller, L. H. 1963, ApJ, 137,280

Wilhelm, R., Beers, T. C., Kriessler, J. R., Pier, J. R., Sommer-Larsen, J., \& Layden, A. C. 1996, in Astronomical Society of the Pacific Conference Series, Vol. 92, Formation of the Galactic Halo...Inside and Out, ed. H. L. Morrison \& A. Sarajedini, 171-+ 
Woolf, V. M., Tomkin, J., \& Lambert, D. L. 1995, ApJ, 453, 660

Woosley, S. E., \& Weaver, T. A. 1995, ApJS, 101, 181 


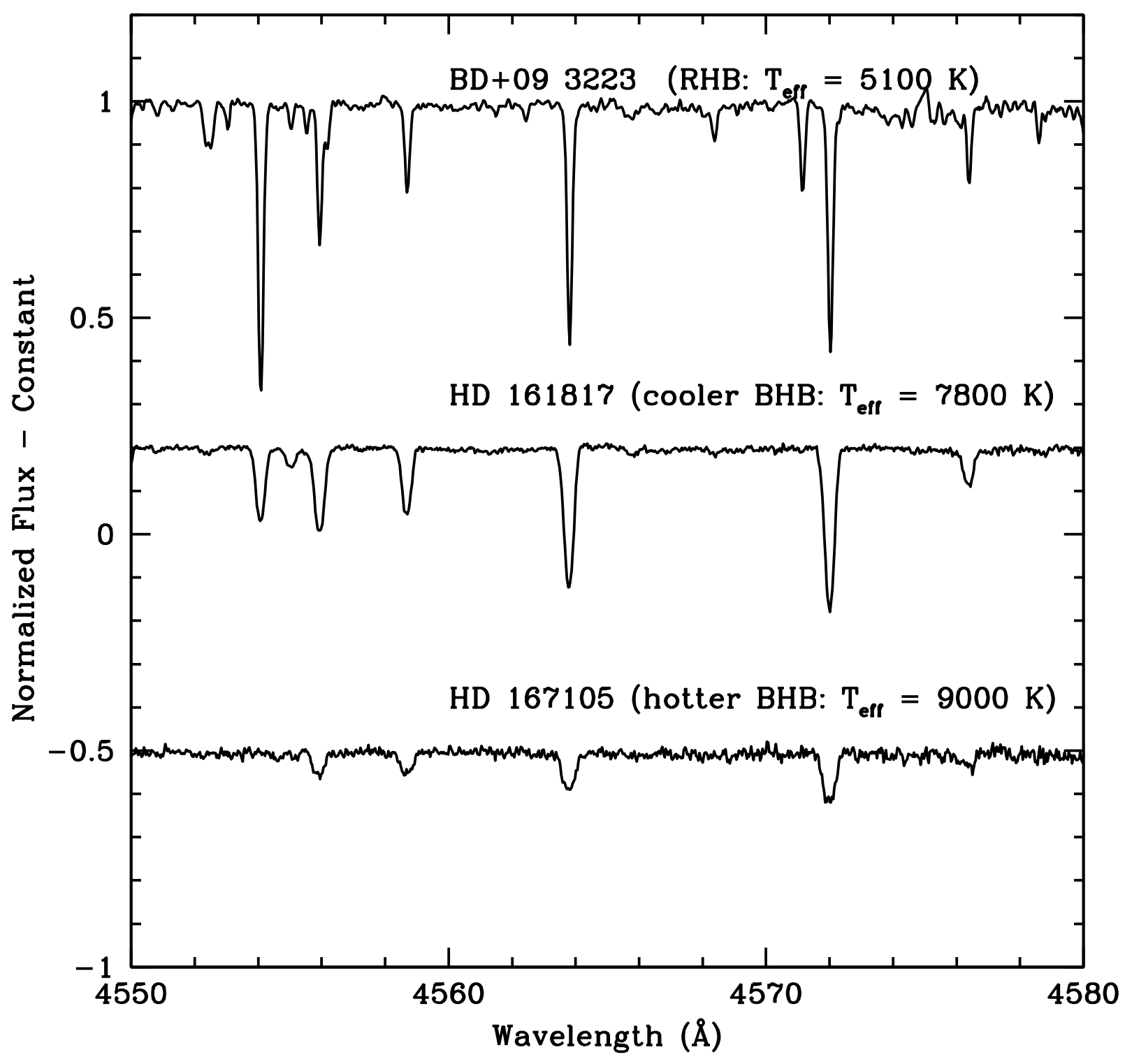

Fig. 1.- Typical reduced, normalized spectra of RHB and BHB stars obtained at McDonald $2.7 \mathrm{~m}$ telescope. Large rotational velocity is seen in hotter BHB stars. 

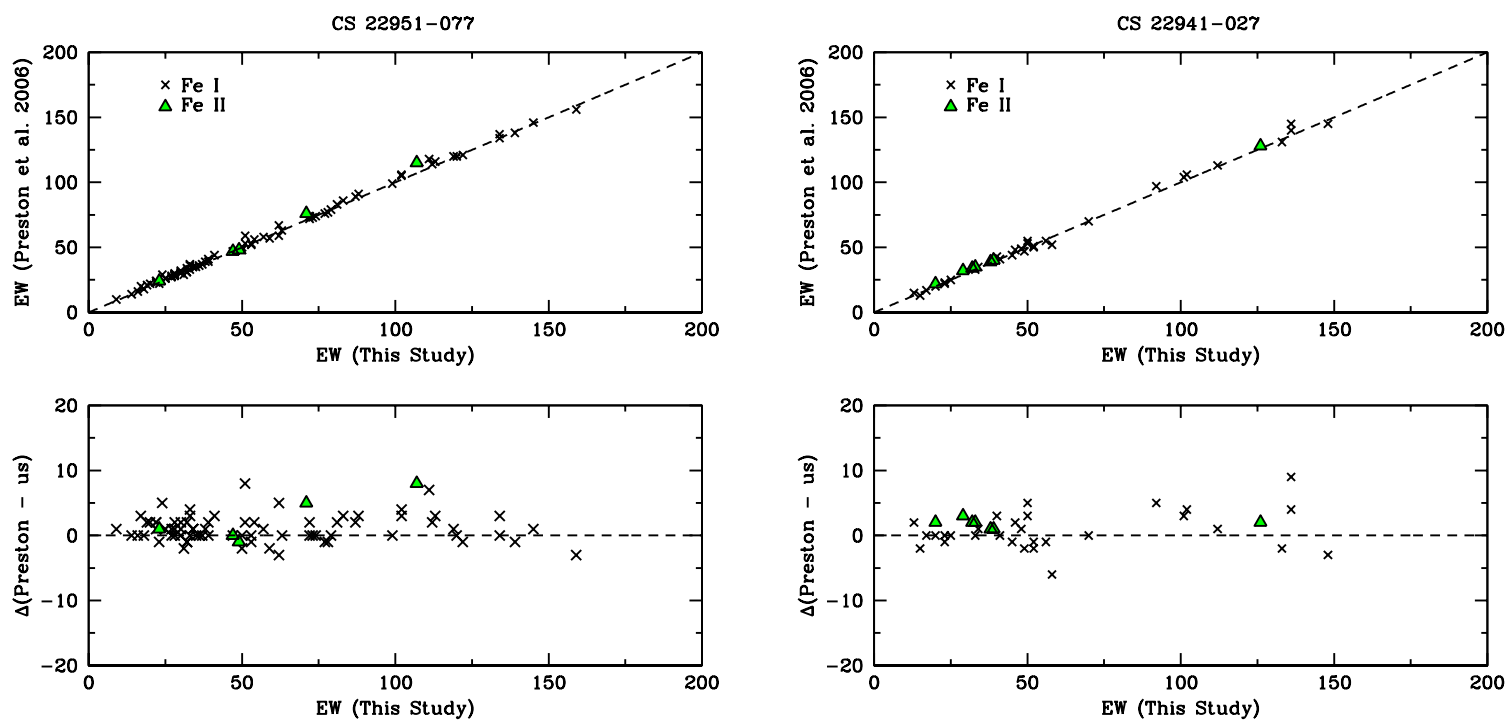

Fig. 2.- Comparisons of our measured Fe I \& II EWs of cooler (CS 22951-077) and hotter (CS 22941-027) MPFRHB stars with Preston et al. (2006a). The top panels show 1:1 comparison of EW measurements. The bottom panels show the difference between our EW measurements and Preston et al. (2006a). The crosses and triangles represent Fe I and Fe II lines, respectively. 

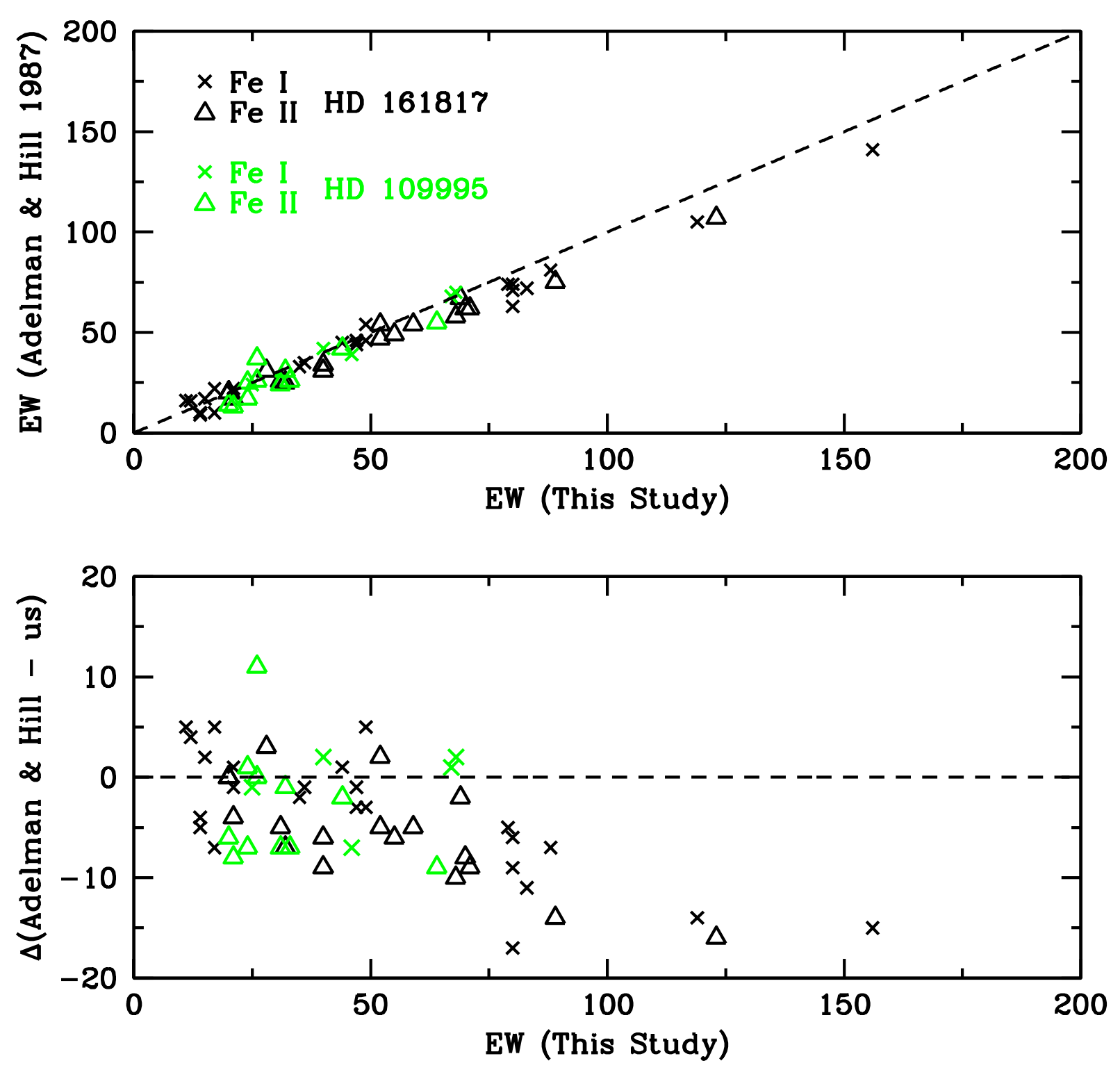

Fig. 3.- Comparisons of our measured Fe I \& II EWs of HD 161817 and HD 109995 with Adelman \& Hill (1987). The top panel shows 1:1 comparison of EW measurement. The bottom panel shows the difference between our EW measurements and Adelman \& Hill (1987). See text for explanation on the large deviation between ours and Adelman \& Hill (1987) measurements. The crosses and triangles represent Fe I and Fe II lines. The green and black correspond to lines measured in HD 109995 and HD 161817, respectively. 


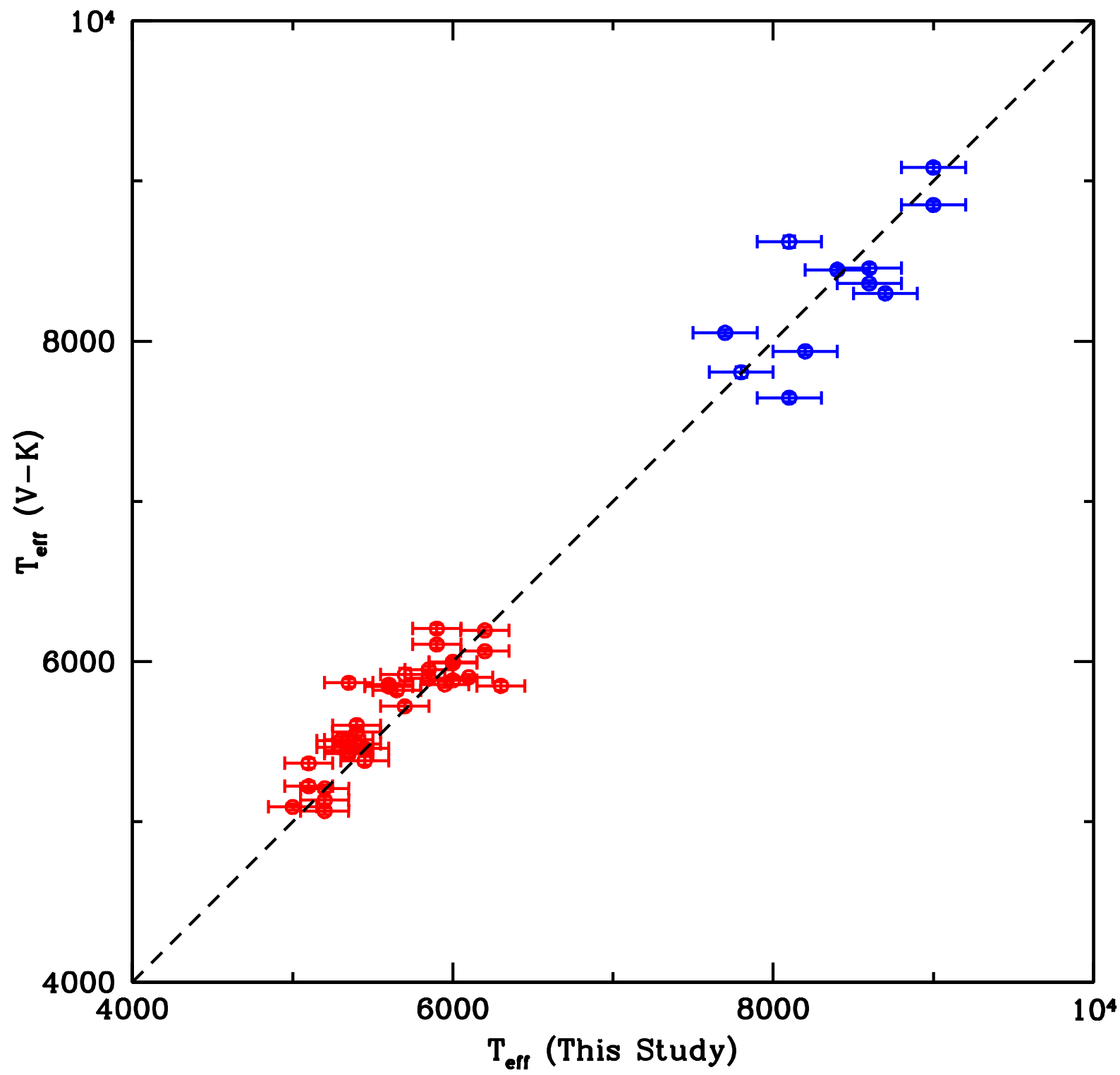

Fig. 4.- Comparison of spectroscopic $T_{\text {eff }}$ with photometric $T_{\text {eff }}$ derived from $(V-K)_{\text {TCS }}$ metallicity-dependent $T_{\text {eff }}$-color formula of Alonso et al. (1999). The error of photometric $T_{\text {eff }}$ is equal to or smaller than the size of the dots. 


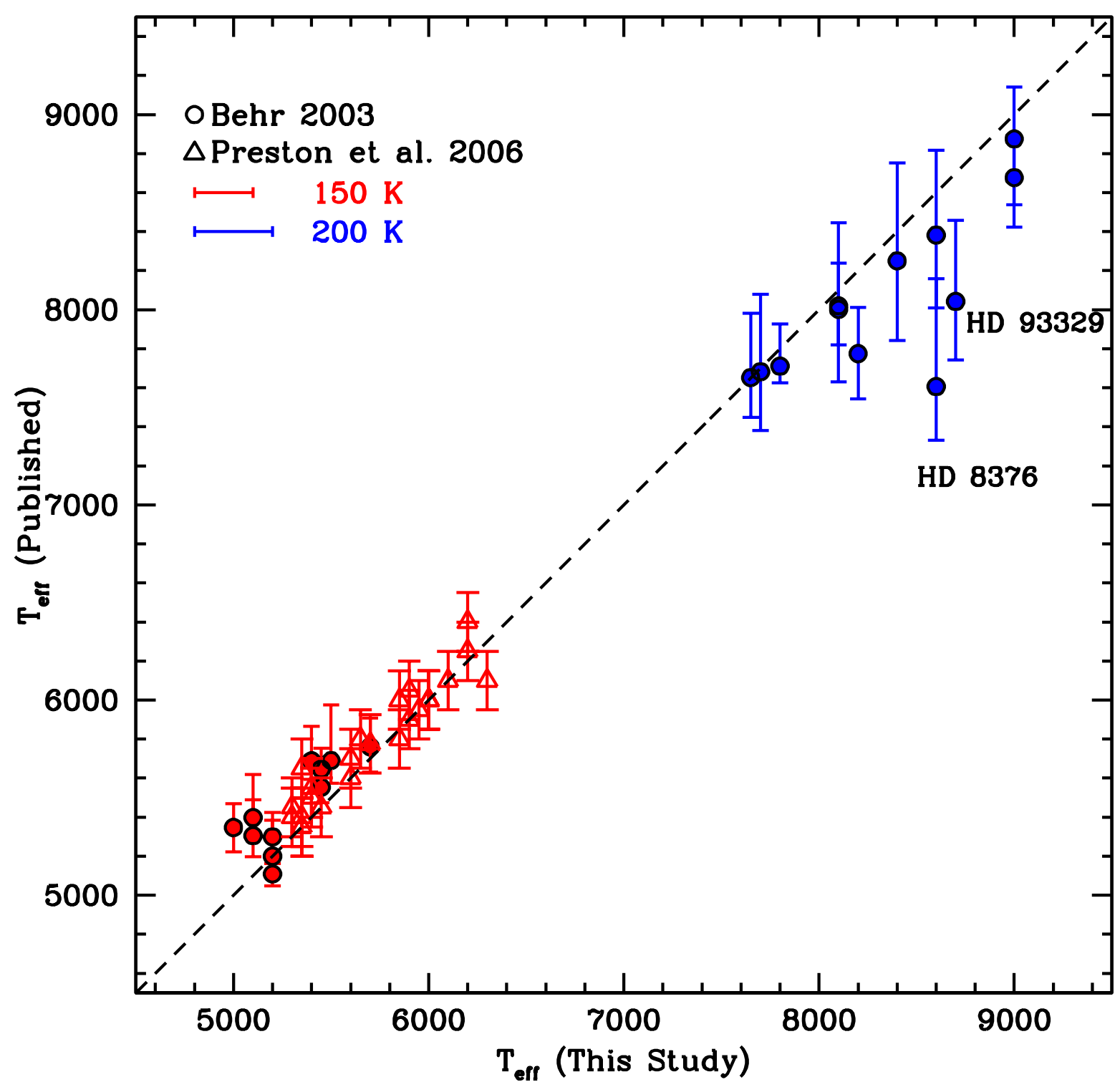

Fig. 5.- Comparison of spectroscopic $T_{\text {eff }}$ derived from this study with $T_{\text {eff }}$ values from Preston et al. (2006a) and Behr (2003b). The triangles and circles represent Preston et al. (2006a) and Behr (2003b) study, respectively. The red and blue colors correspond to RHB and BHB stars. For clarity in the figure, we do not plot error bars from our work for each star, but instead indicate typical $T_{\text {eff }}$ uncertainties for this study, $150 \mathrm{~K}$ and $200 \mathrm{~K}$ for RHB and BHB stars. Comparison of BHB stars can only be made with Behr (2003b). 


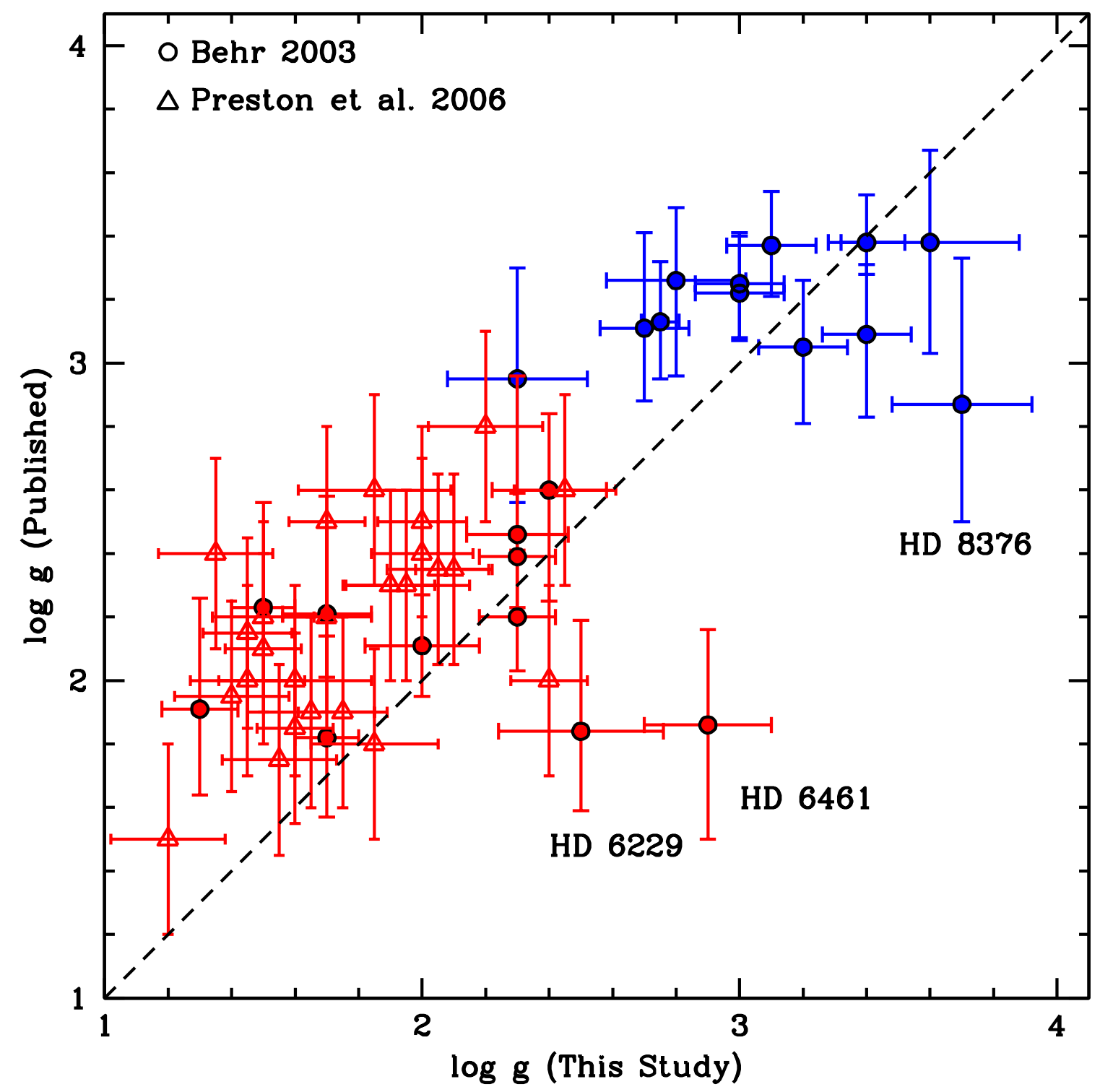

Fig. 6.- Comparison of spectroscopic $\log g$ derived from this study with $\log g$ derived by Preston et al. (2006a) and Behr (2003b). The triangles and circles represent Preston et al. (2006a) and Behr (2003b) study, respectively. The red and blue colors correspond to RHB and BHB stars. 


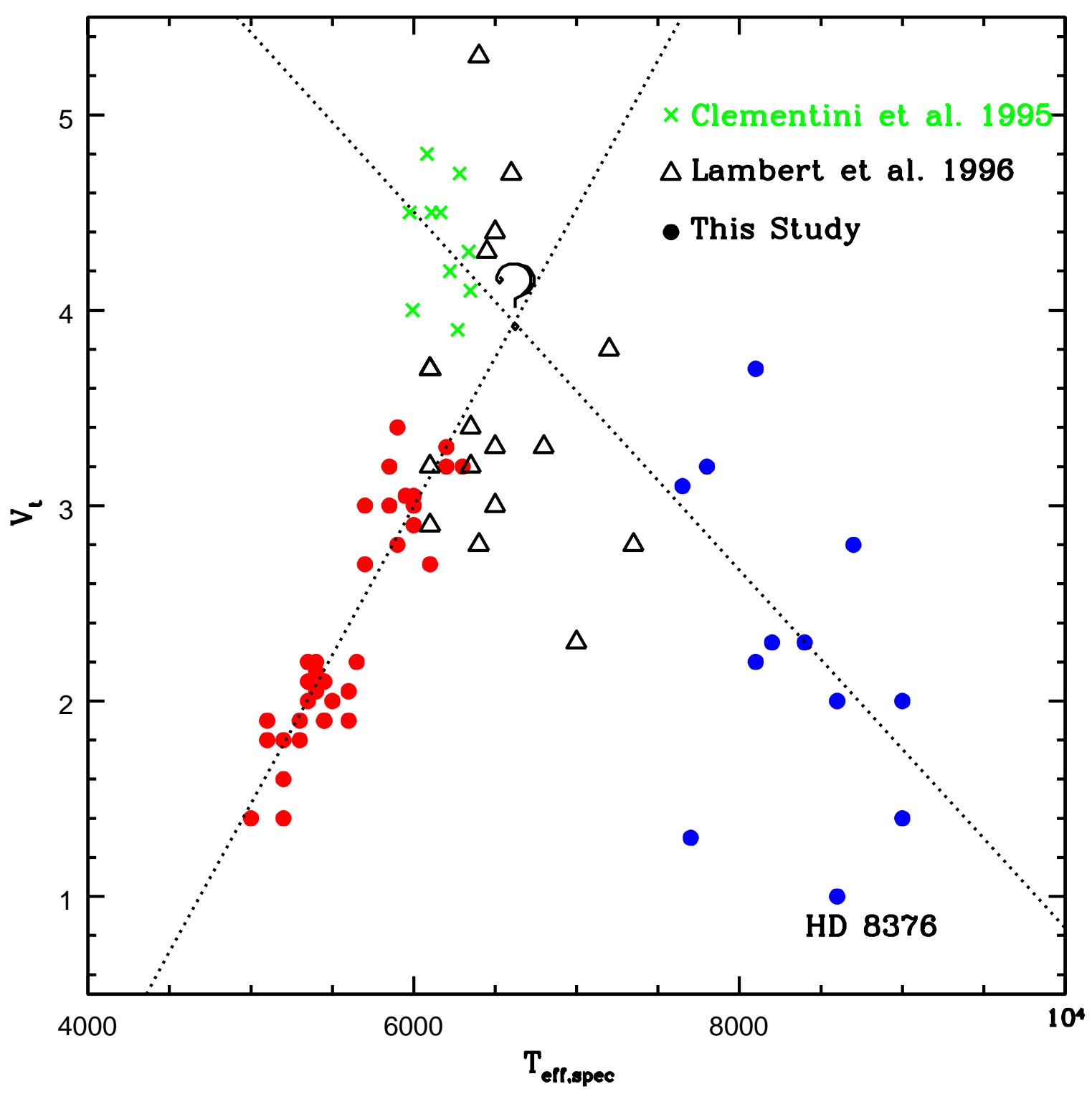

Fig. 7.- The correlation and anti-correlation between $v_{\mathrm{t}}$ and $T_{\text {eff }}$ for RHB and BHB stars. Linear least square equations were fitted to all the RHB stars and BHB stars, excluding HD 8376. The crosses and open triangles represent the $v_{\mathrm{t}}$ and $T_{\text {eff }}$ of RR Lyrs studies by Clementini et al. (1995) and Lambert et al. (1996), respectively. The readers are warned that there is no correlation in the RR Lyr IS region and beyond the intersection of dashed lines, where question mark is marked. 
Light odd $-\mathrm{Z}$ and $\alpha$-elements

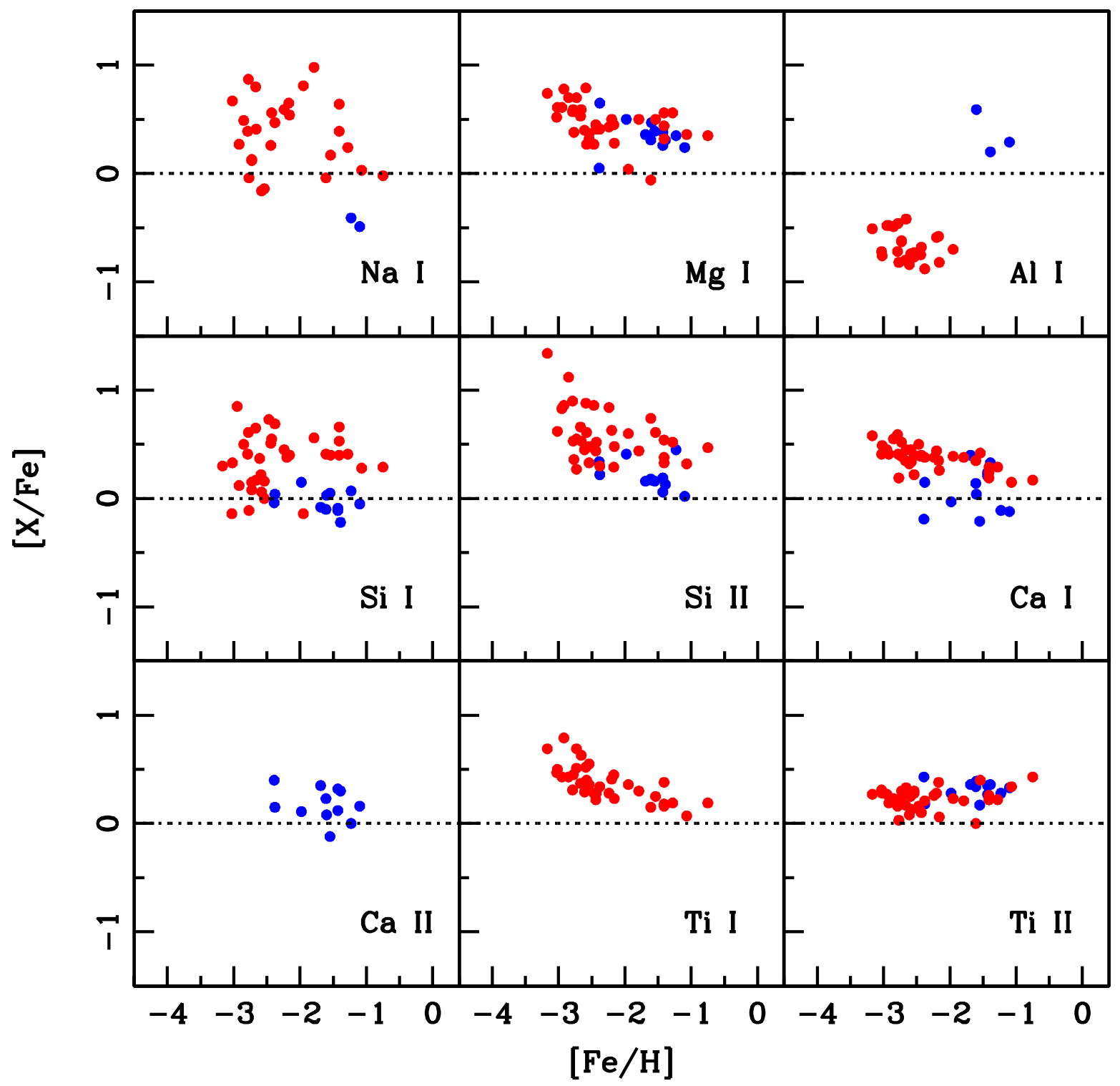

Fig. 8.- Abundance ratios of odd- $Z$ and $\alpha$-elements as a function of metallicity. NLTE corrections applied to Na I, Al I, Si I \& Si II as described in text. The red and blue dots represent RHB and BHB stars. 
Fe-peak elements

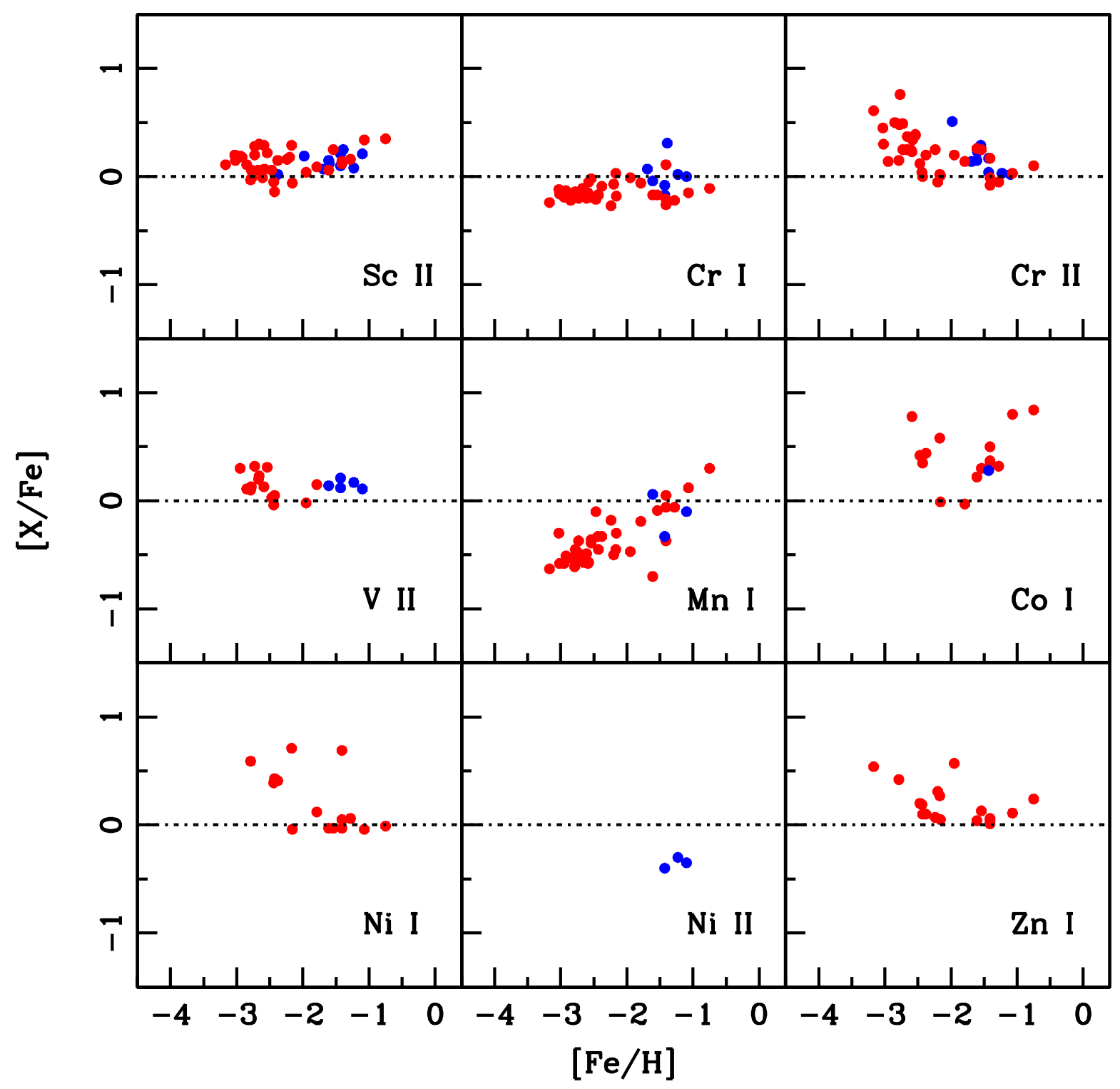

Fig. 9.- Abundance ratios of Fe-peak elements as a function of metallicity. The red and blue dots represent RHB and BHB stars. 


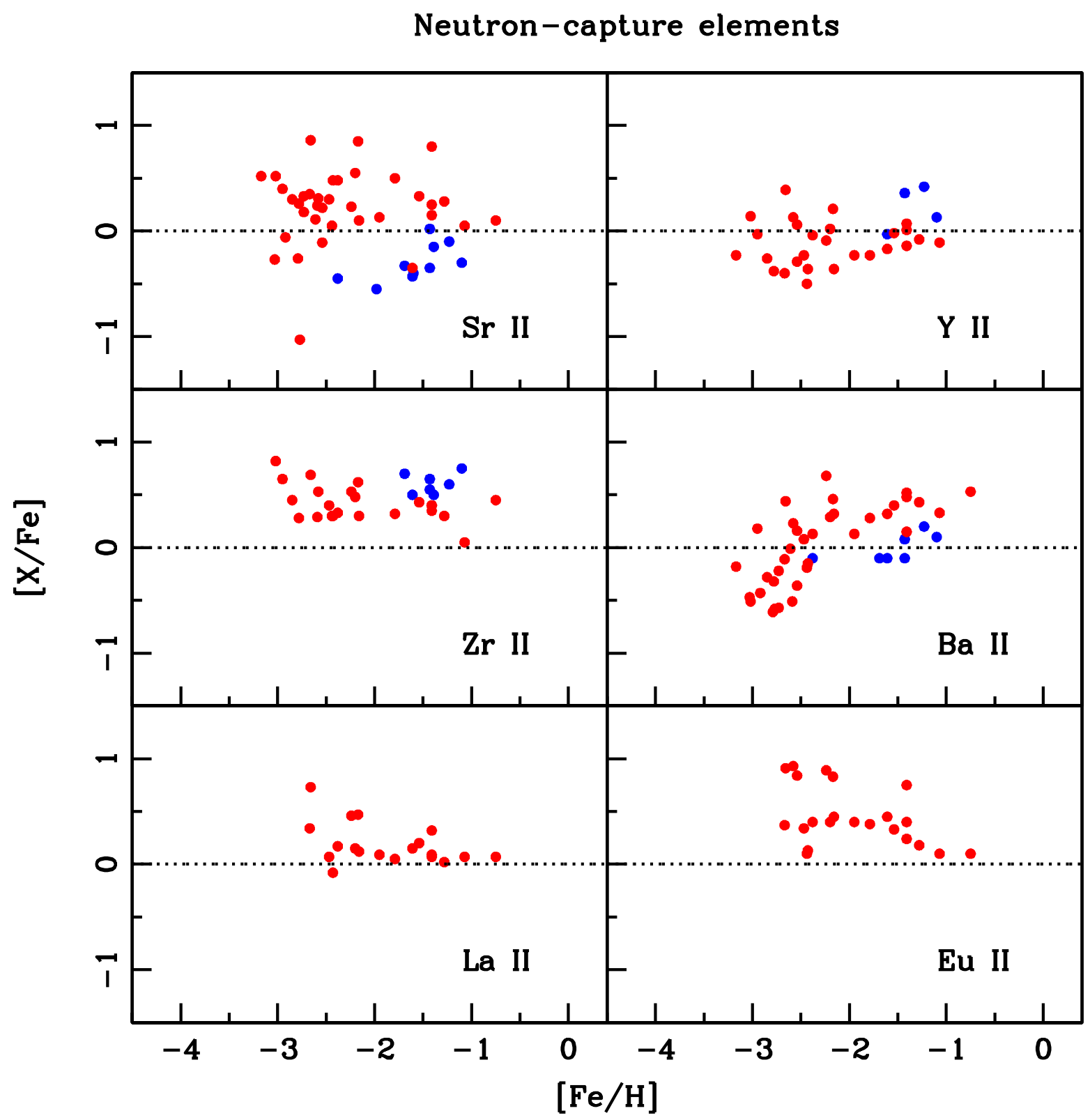

Fig. 10.- Abundance ratios of neutron-capture elements as a function of metallicity. The red and blue dots represent RHB and BHB stars. 
Light odd $-\mathrm{Z}$ and $\alpha$-elements

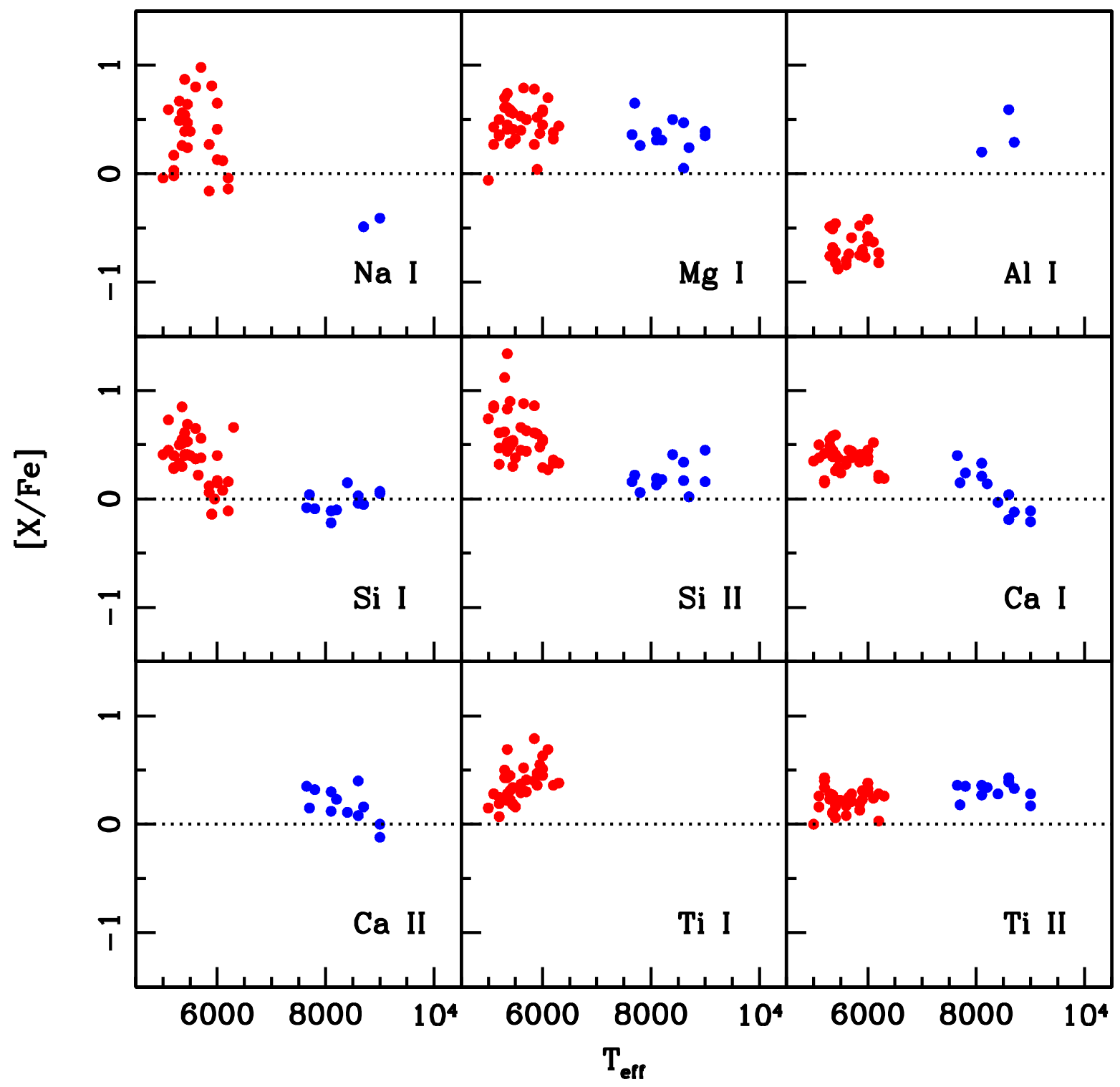

Fig. 11. - Abundance ratios of odd- $Z$ and $\alpha$-elements as a function of spectroscopic $T_{\text {eff }}$. NLTE corrections applied to Na I, Al I, Si I \& Si II as described in text. The red and blue dots represent RHB and BHB stars. 
Fe-peak elements

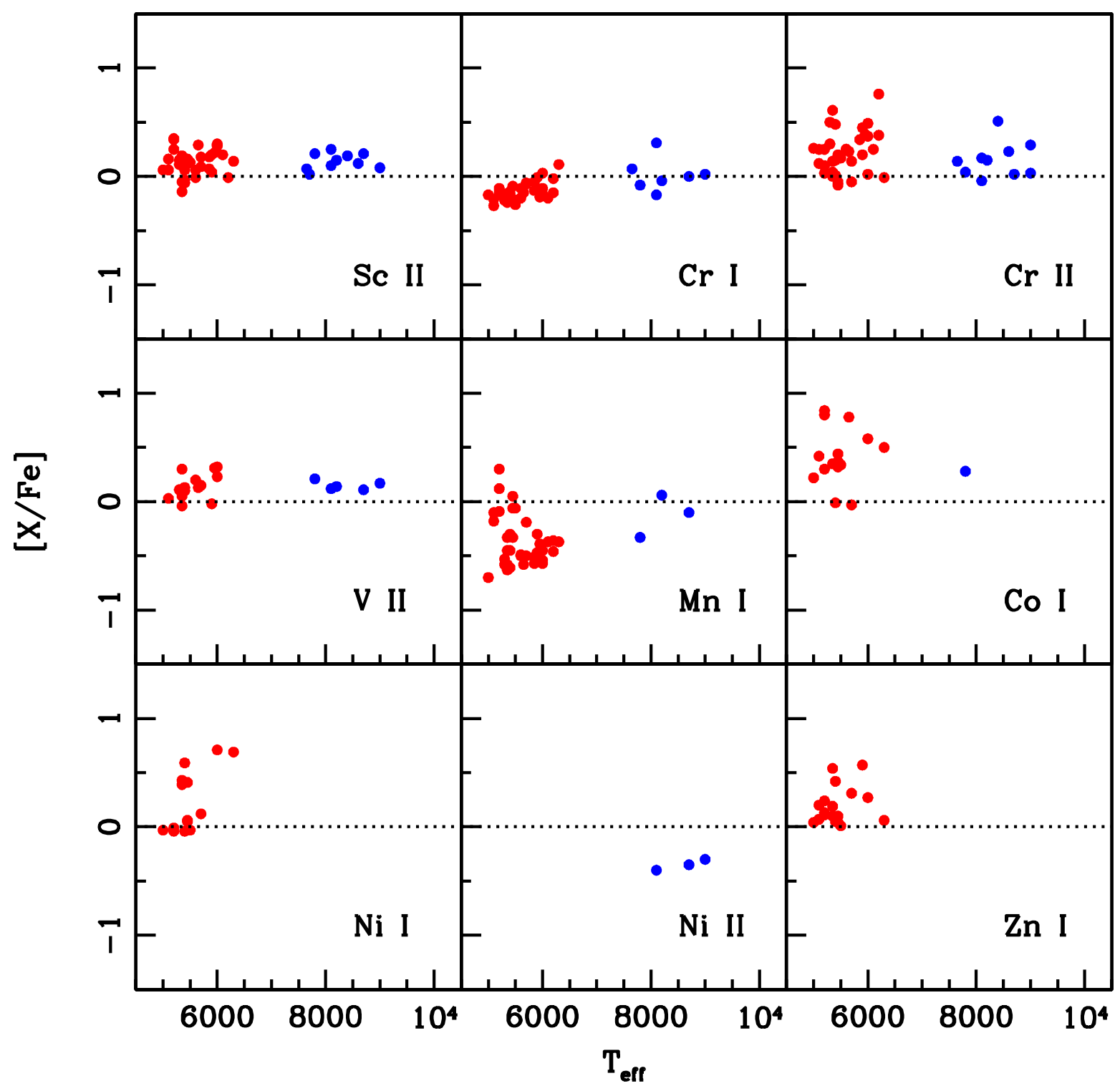

Fig. 12.- Abundance ratios of Fe-peak elements as a function of spectroscopic $T_{\text {eff }}$. The red and blue dots represent RHB and BHB stars. 


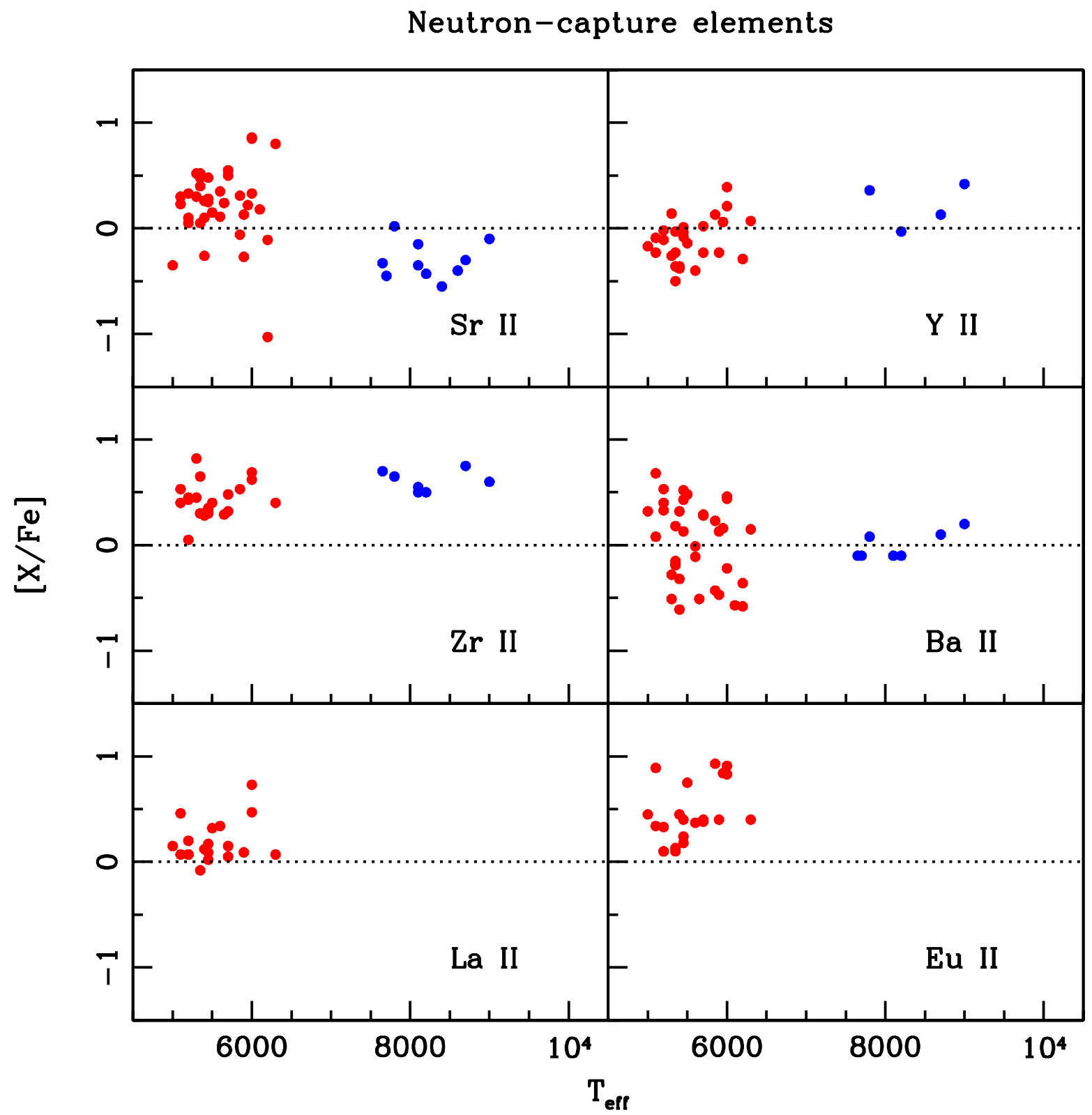

Fig. 13.- Abundance ratios of neutron-capture elements as a function of spectroscopic $T_{\text {eff }}$. The red and blue dots represent RHB and BHB stars. 


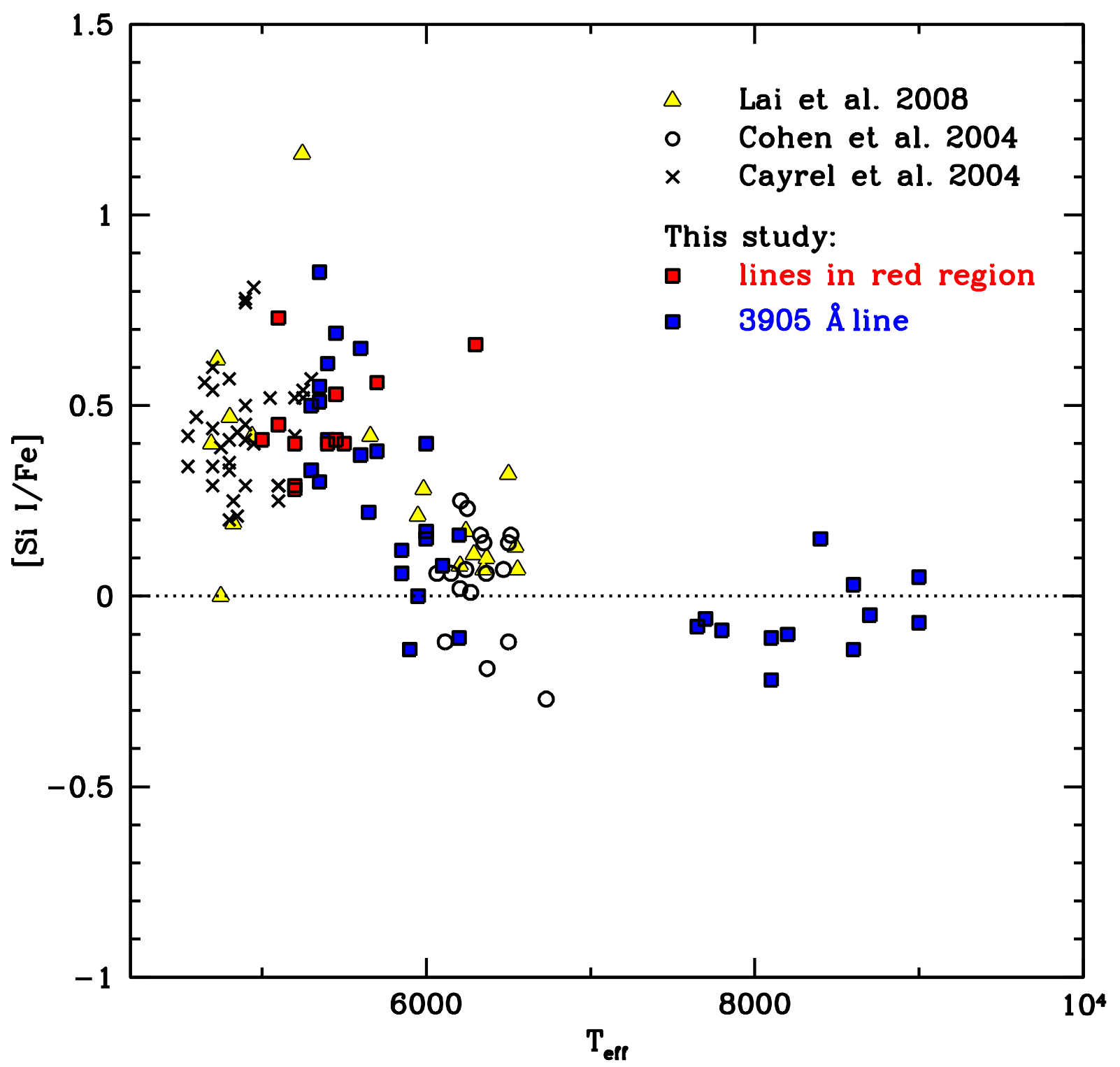

Fig. 14.- Abundance ratios of $[\mathrm{Si} \mathrm{I} / \mathrm{Fe}]$ vs spectroscopic $T_{\text {eff }}$, with the addition of data of very metal-poor stars giants from Cayrel et al. (2004) (crosses), low-luminosity near-turnoff stars from Cohen et al. (2004) (open circles) and stars in different evolutionary states from Lai et al. (2008) (yellow triangles). The derived $[\mathrm{Si} \mathrm{I} / \mathrm{Fe}]$ in this study is represented by filled rectangles. NLTE correction applied to $[\mathrm{Si} / \mathrm{Fe}]$ as described in text. The red and blue colors represent Si I lines in red spectral region and $3905 \AA$ line, respectively. 


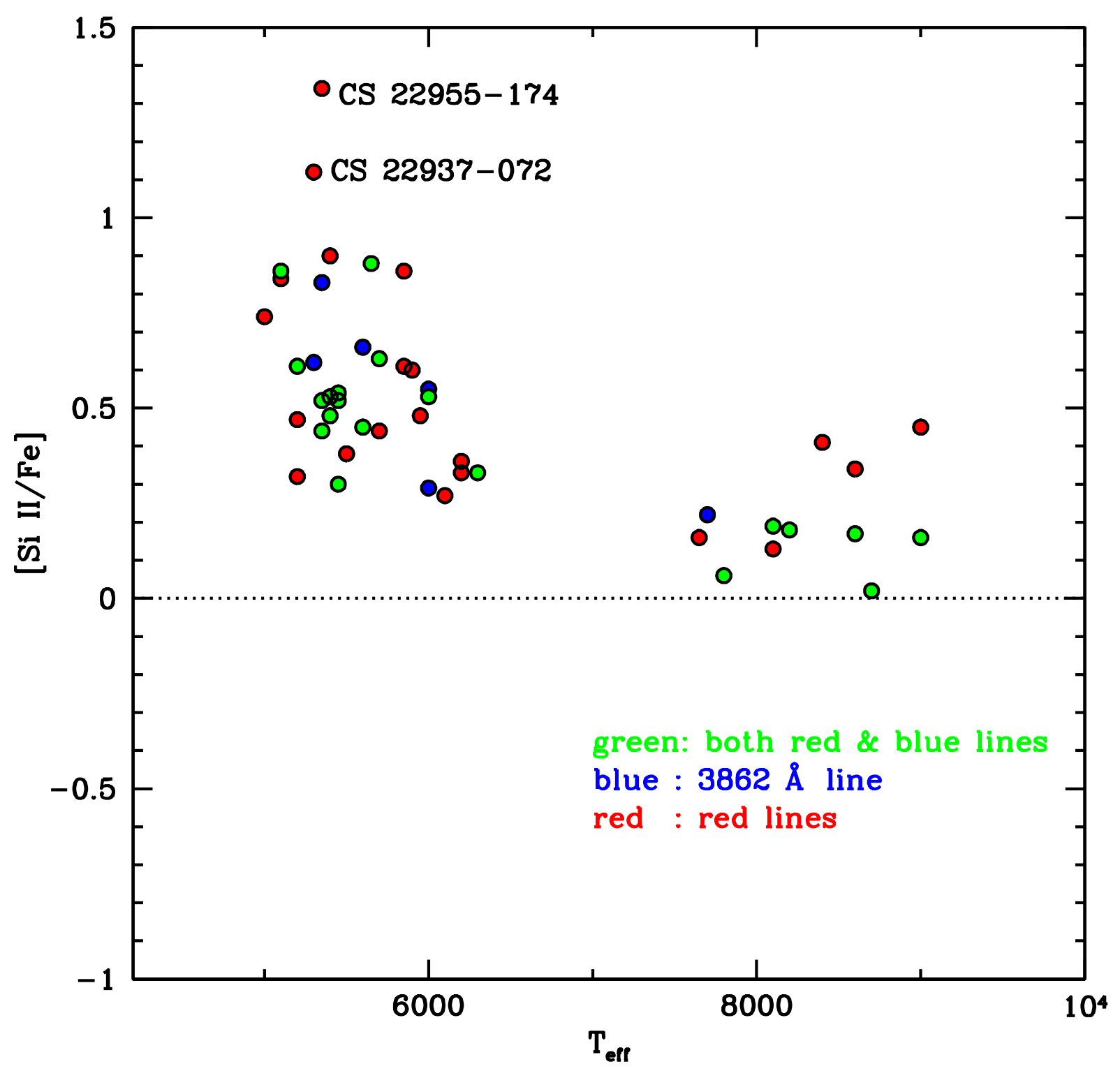

Fig. 15.- Abundance ratios of $[\mathrm{Si} \mathrm{II} / \mathrm{Fe}]$ vs spectroscopic $T_{\text {eff }}$. NLTE correction applied to $[\mathrm{Si} \mathrm{II} / \mathrm{Fe}]$ as described in text. The colors represent the usage of lines in different spectral regions for EW analysis. 


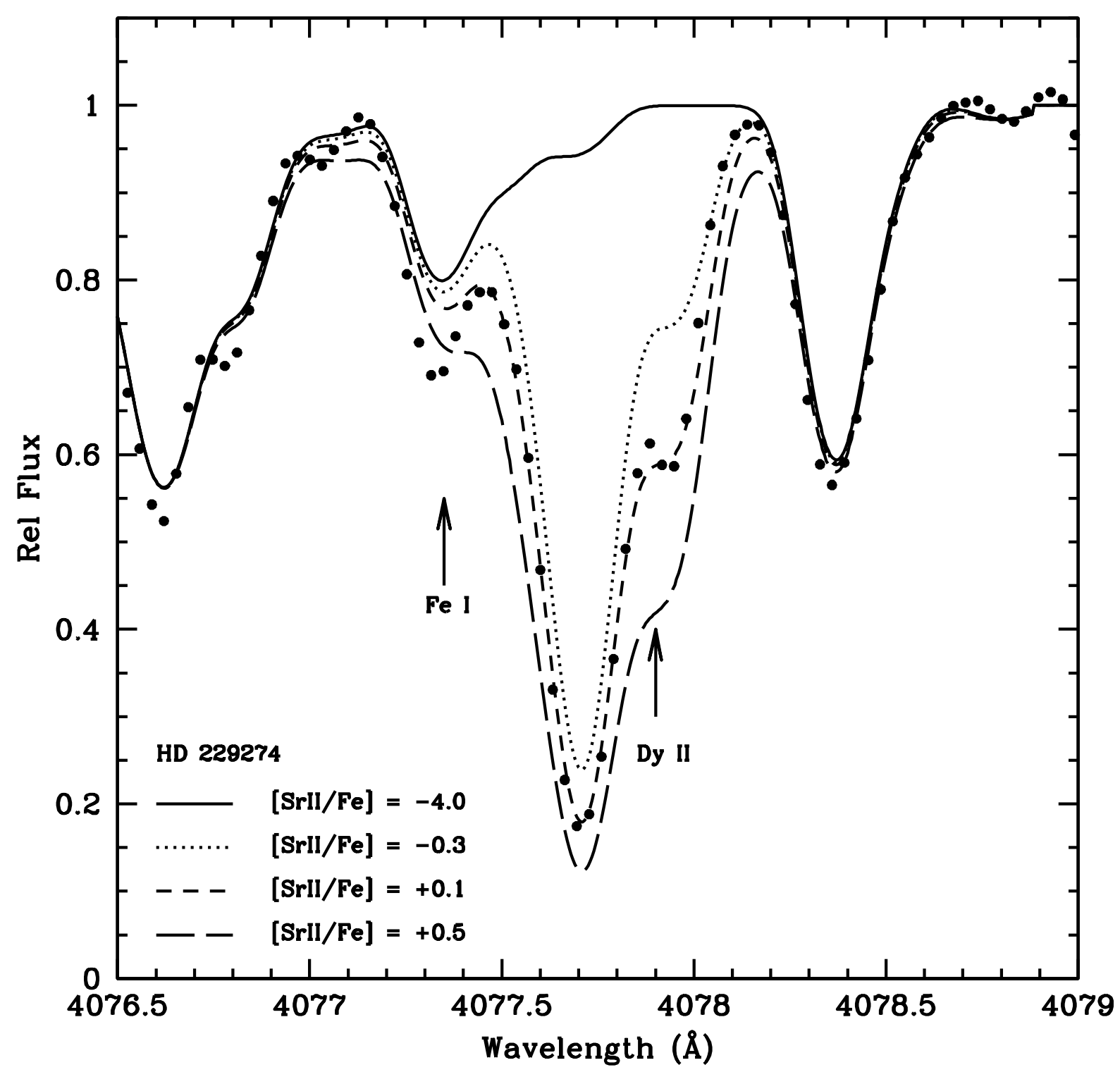

Fig. 16.- An example of synthesized Sr II $4077 \AA$ line superimposed on the observed spectrum. The assumed Fe abundance is the same as the metallicity used in the stellar parameters. The solid and medium dashed lines represent no $\mathrm{Sr}$ contribution and derived $\mathrm{Sr}$ abundance ratio for this line. The dotted and long dashed lines are \pm 0.4 dex of derived $\mathrm{Sr}$ abundance ratio. 


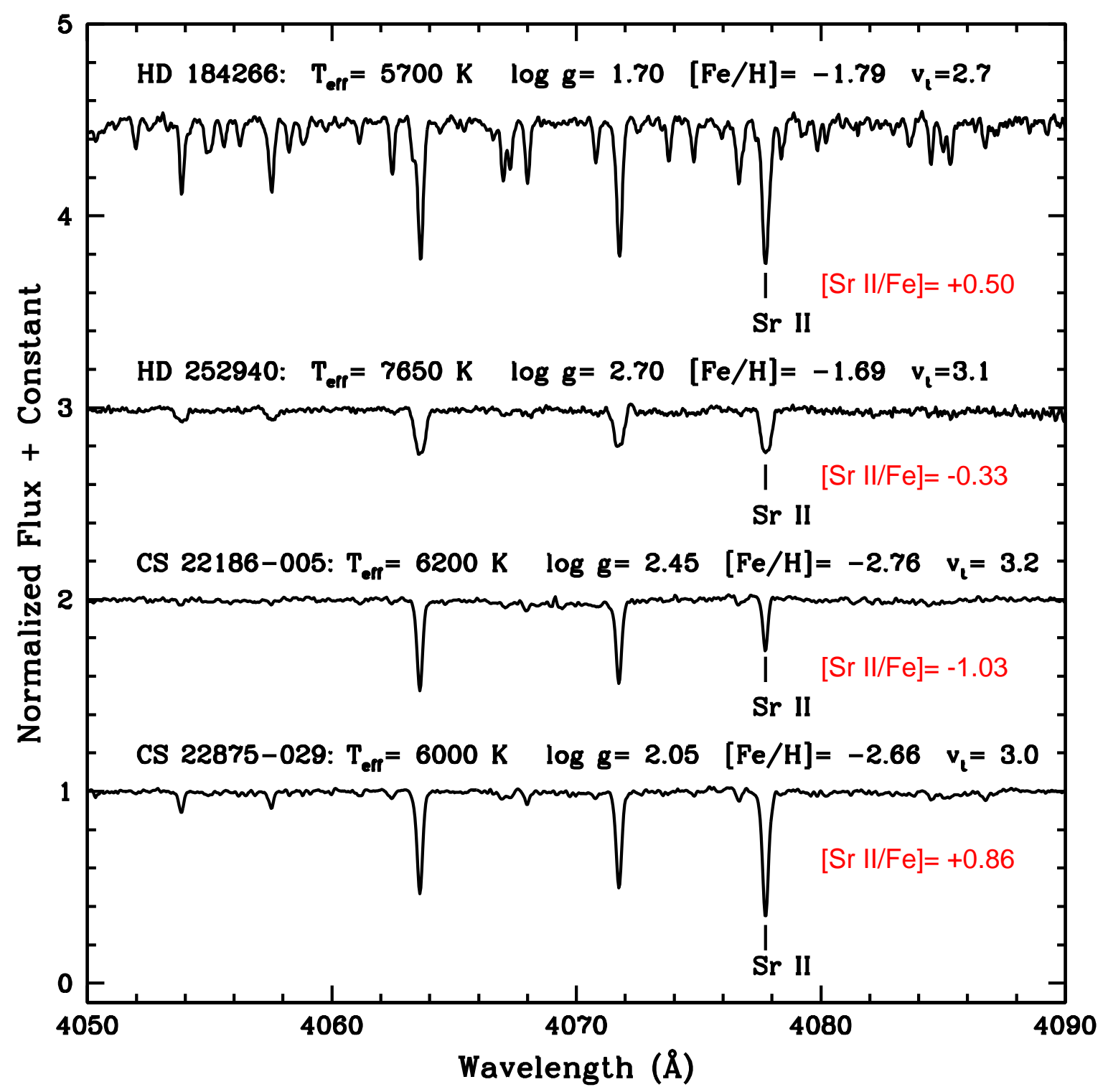

Fig. 17.- The top two spectra show the different Sr II line strength between RHB and BHB stars. As shown, Sr II line in BHB stars is not as strong as in RHB stars. The bottom two stars posses similar stellar parameters but show different line strength in Sr II line. 


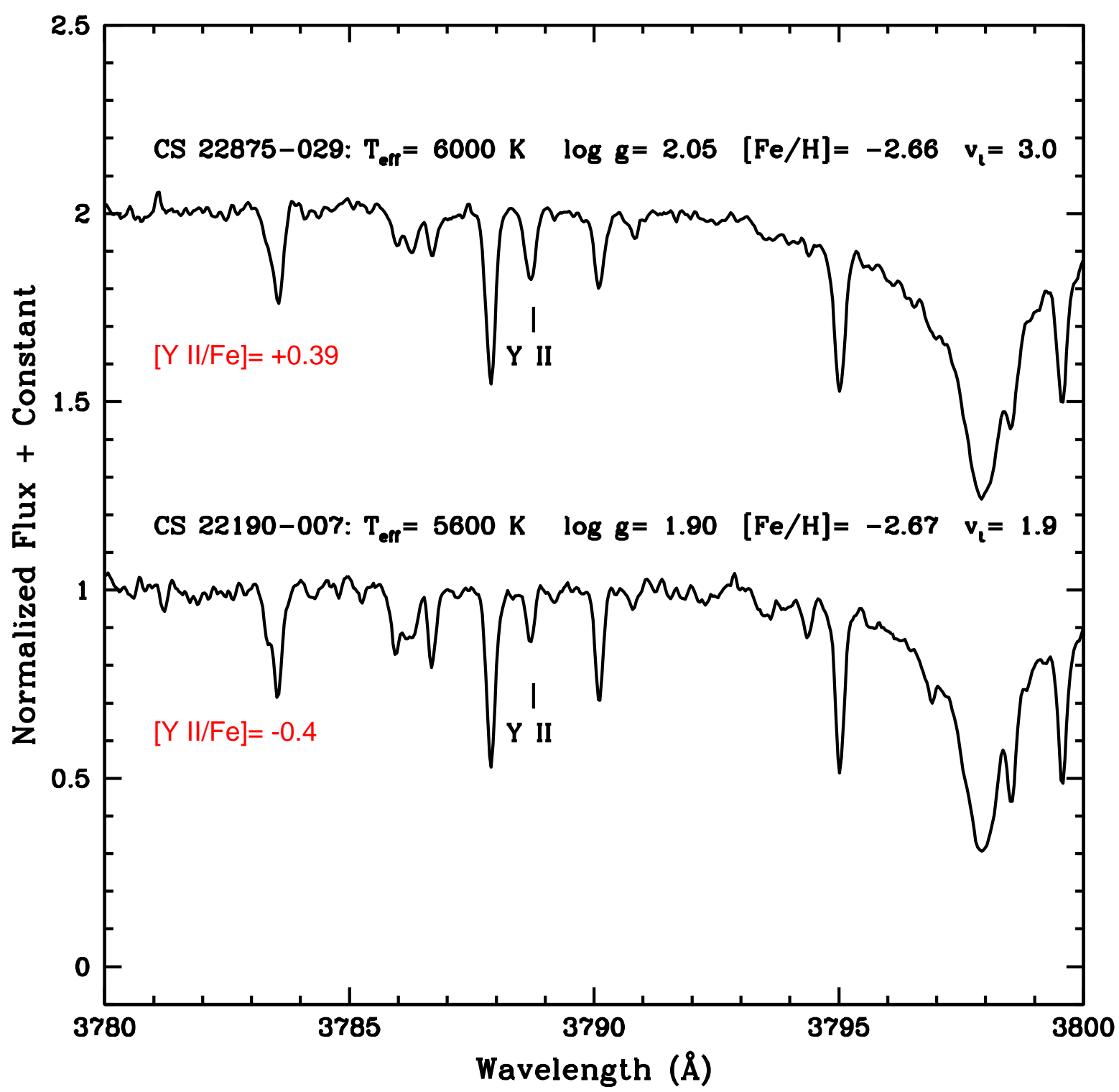

Fig. 18. - Comparison of Y II line strength of stars with similar $[\mathrm{Fe} / \mathrm{H}]$. The low and high Y II abundance ratios of these two stars contribute to the scatter of [Y II/Fe] vs $[\mathrm{Fe} / \mathrm{H}]$. 


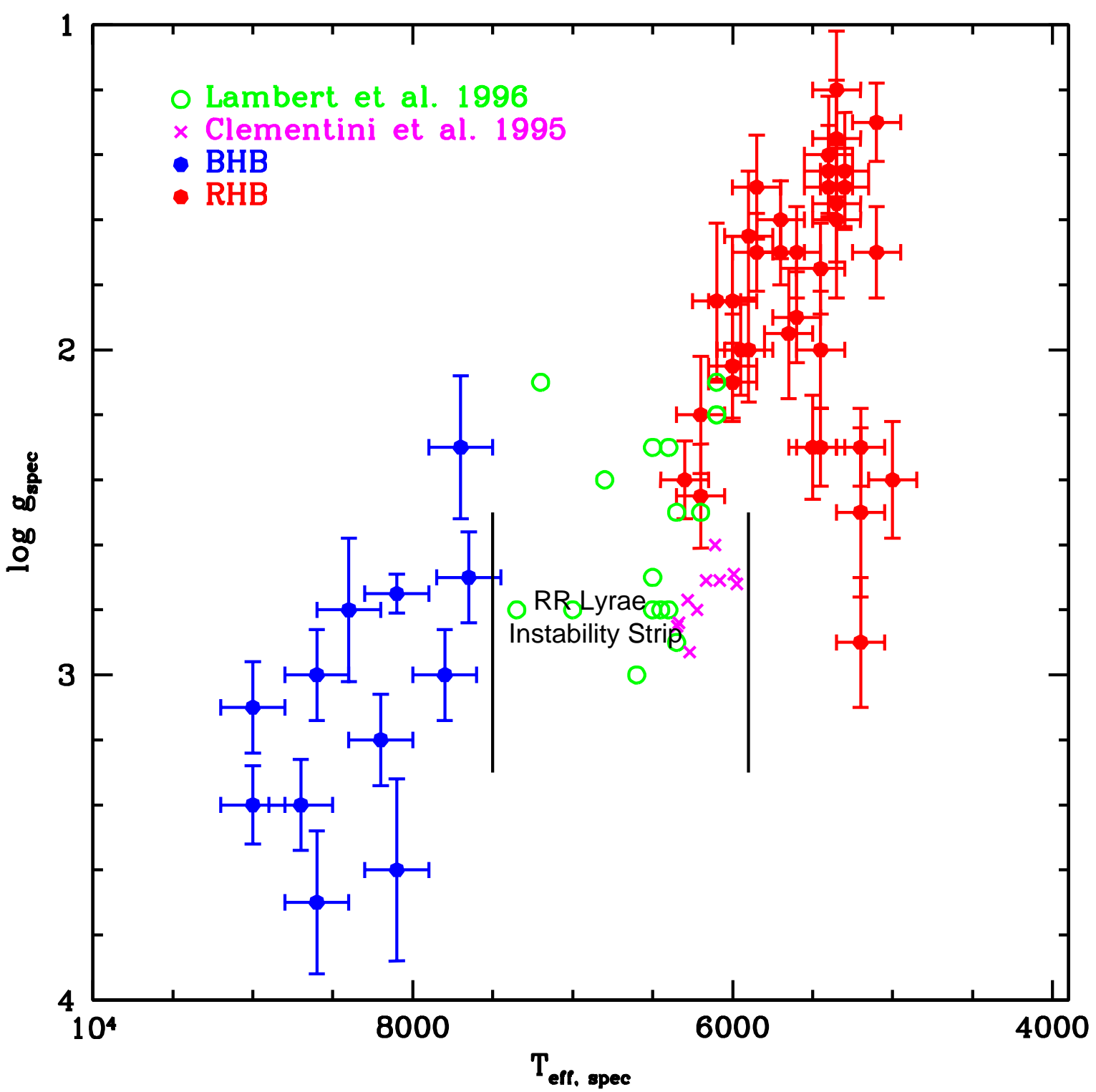

Fig. 19.- The spectroscopic $T_{\text {eff }}$ and $\log g$ of our RHB and BHB stars (red and blue dots), and $T_{\text {eff }}$ and $\log g$ of field RR Lyraes from Lambert et al. 1996 and Clementini et al. 1995) (green open circles \& magenta crosses) on the $T_{\text {eff }}-\log g$ plane. 


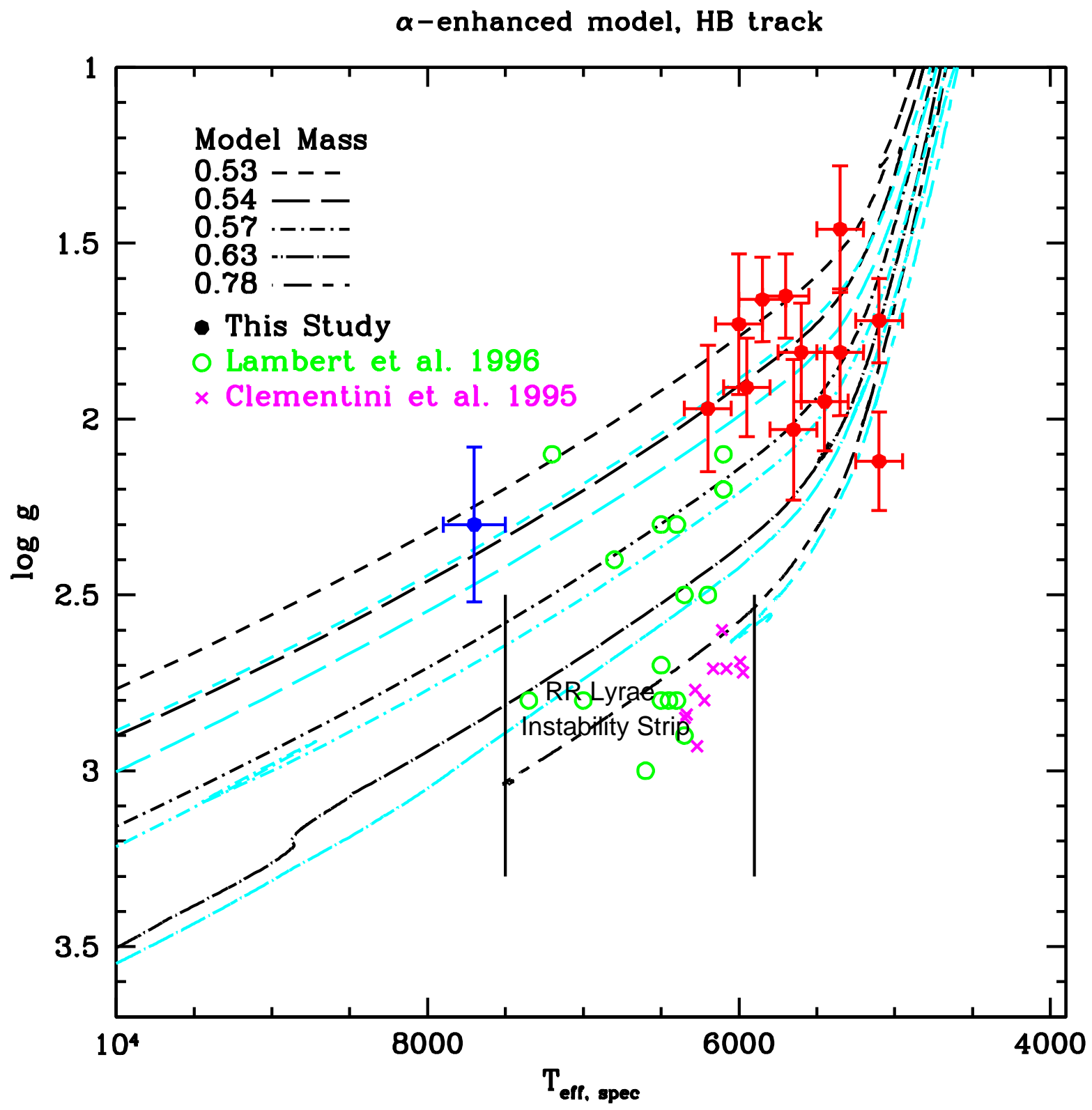

Fig. 20.- The spectroscopic $T_{\text {eff }}$ and photometric/spectroscopic $\log g$ of a set of our RHB and BHB stars (red and blue dots) overlaid on $\alpha$-enhanced HB tracks of $[\mathrm{M} / \mathrm{H}]=-1.79$, $Z=0.0003, Y=0.245$ (black) and $[\mathrm{M} / \mathrm{H}]=-2.27, Z=0.0001, Y=0.245$ (cyan). These HB tracks were used to derive the masses of this set of HB stars. The $T_{\text {eff }}$ and $\log g$ of field RR Lyraes are from Lambert et al. 1996 and Clementini et al. 1995 (green open circles \& magenta crosses). 


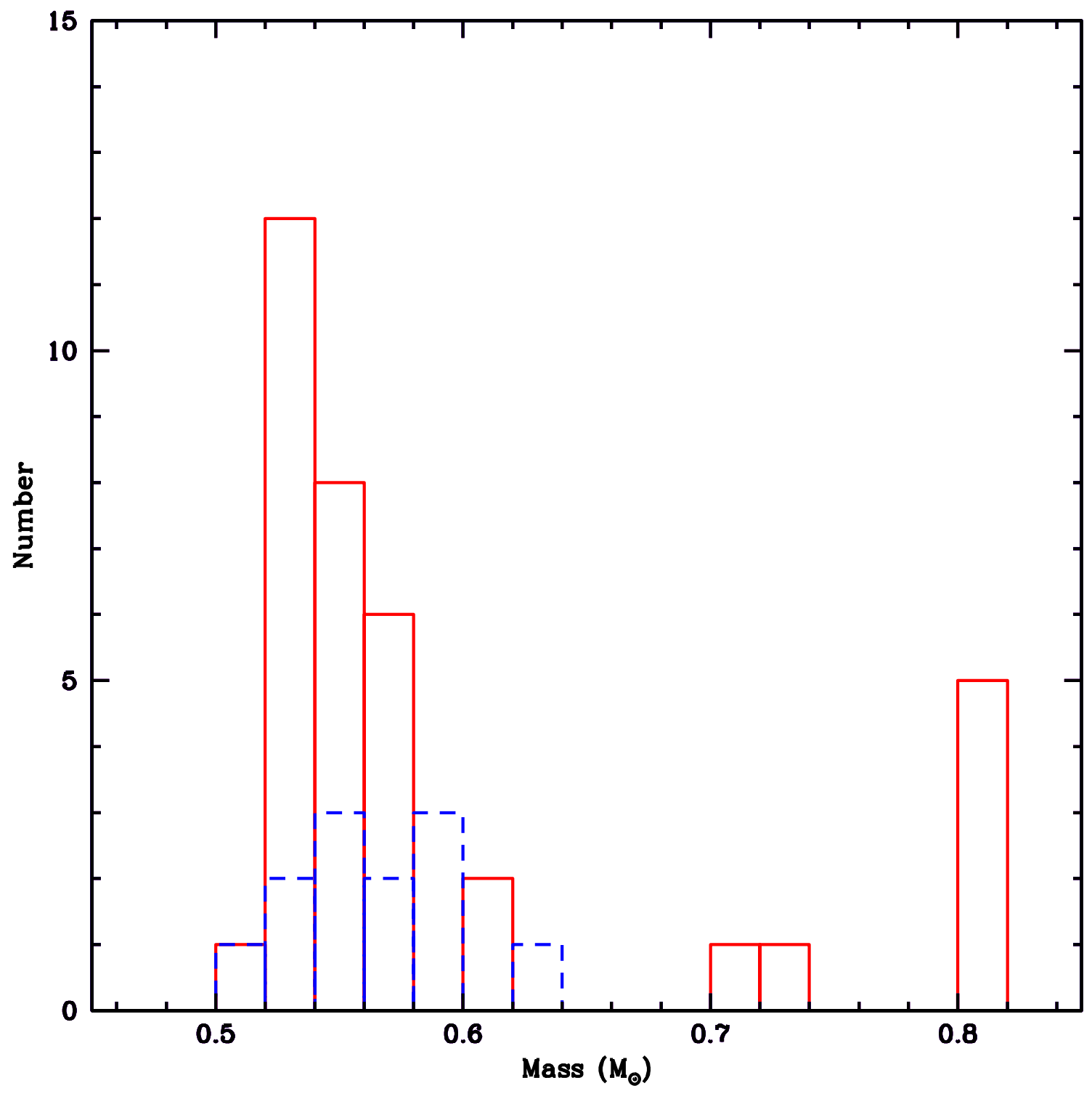

Fig. 21. - The red (solid) and blue (dashed) histograms represent the estimated RHB and BHB masses. The mean masses for RHB and BHB stars are $0.59 M_{\odot}$ and $0.56 M_{\odot}$. Excluding the upper mass limit RHB stars $\left(M>0.7 M_{\odot}\right)$, the mean masses are $0.56 M_{\odot}$ for both RHB and BHB stars. The median masses for RHB and BHB stars are $0.54 M_{\odot}$ and $0.56 M_{\odot}$, respectively. 

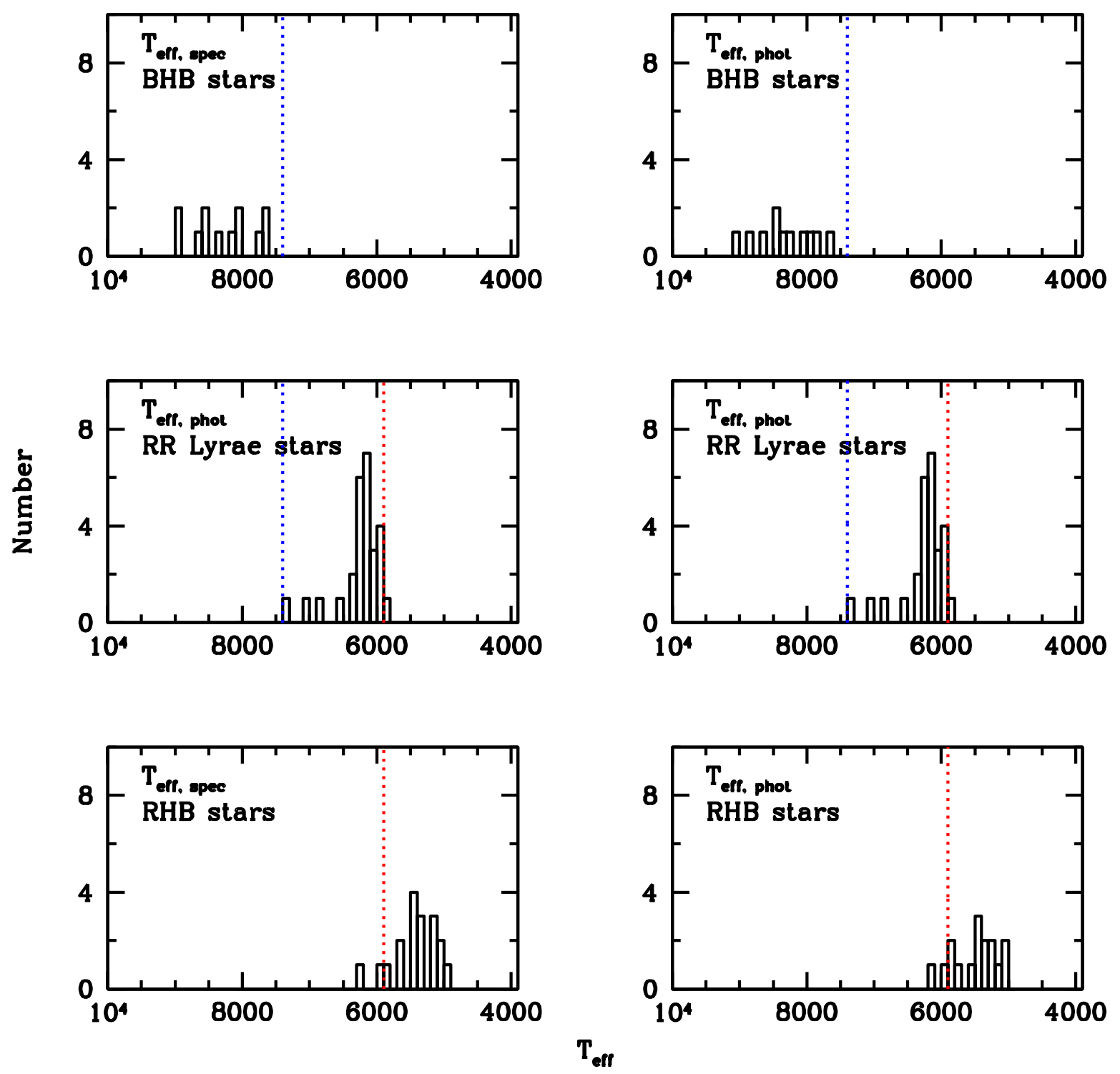

Fig. 22. - The top and bottom panels show the histograms of spectroscopic and photometric $T_{\text {eff }}$ of BHB and RHB stars. The middle panels (same) are the photometric $T_{\text {eff }}$ of field RR Lyr stars extracted from Lambert et al. (1996) and Clementini et al. (1995). The red and blue dotted lines represent the estimated fundamental red and blue edges of field RR Lyr IS for $[\mathrm{Fe} / \mathrm{H}]>-2.5$. 
Light odd-Z and $\alpha$-elements

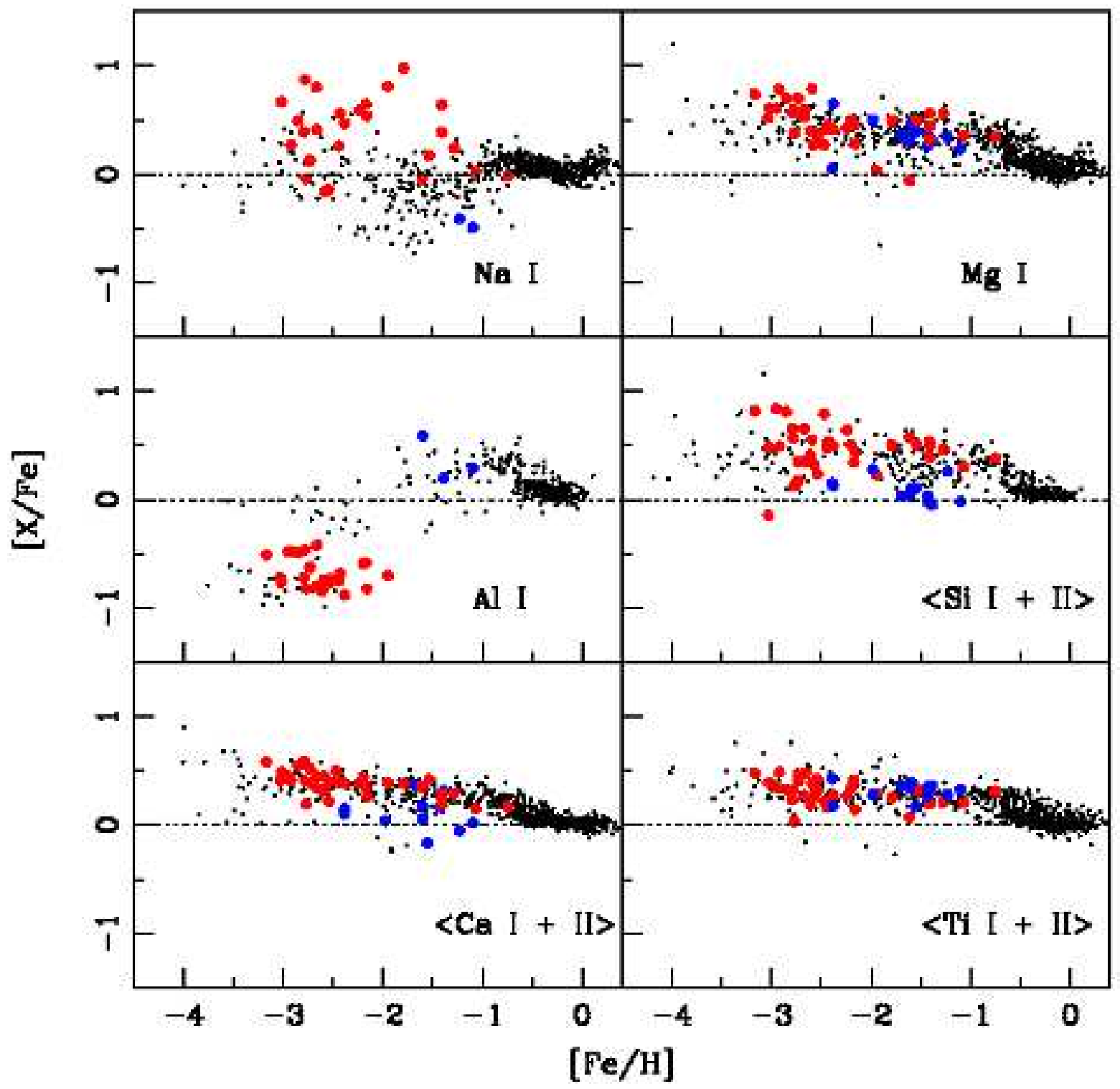

Fig. 23.- Abundance ratios of light odd-Z and $\alpha$-elements in this study superimposed on the data assembled by Venn et al. (2004) and us. Mean of neutral and ionized species are used for comparisons. NLTE corrections applied to Na I, Al I, Si I \& Si II for our HB stars. The red and blue dots correspond to RHB and BHB stars. 


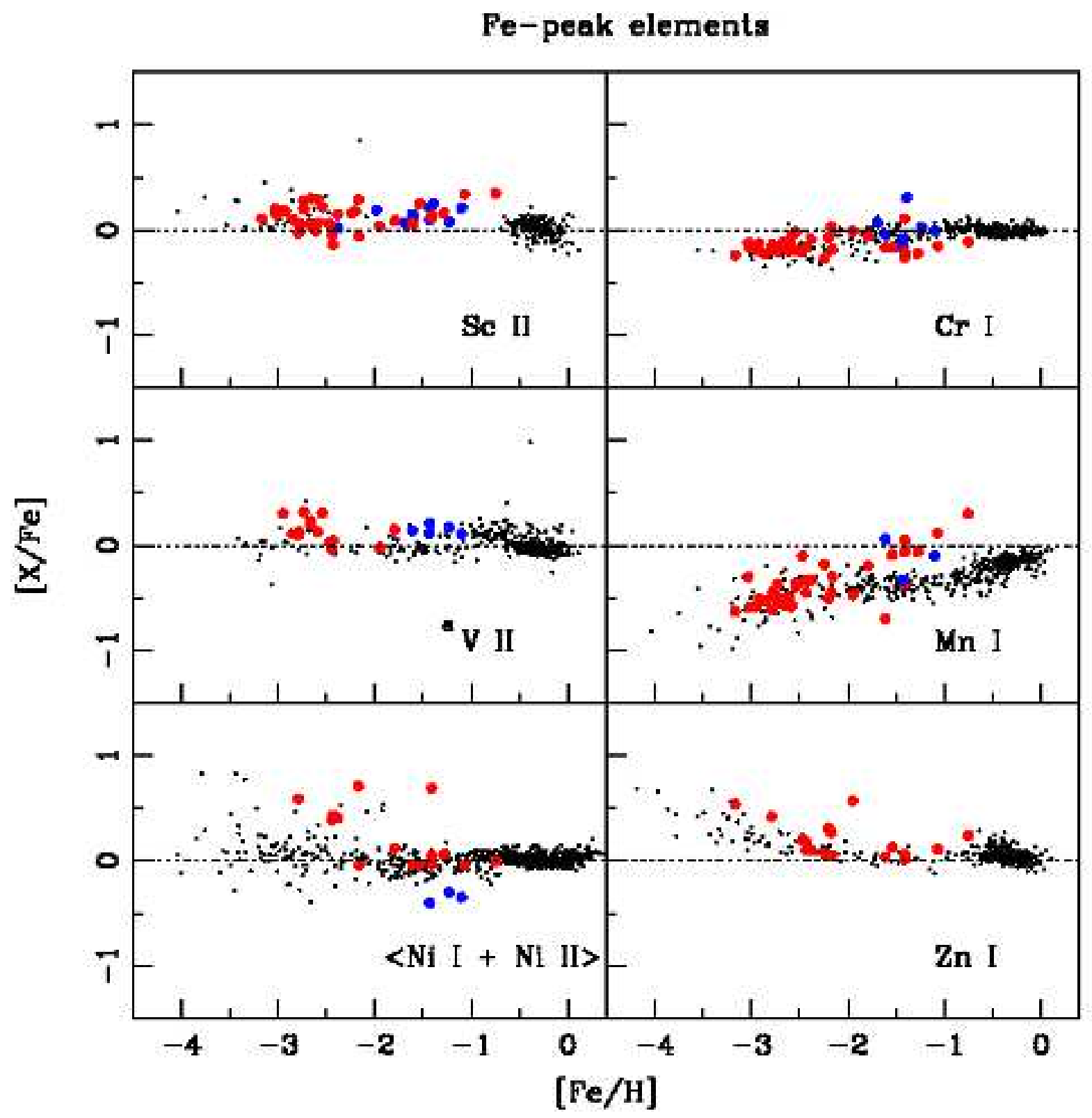

Fig. 24.- Same as Figure 23, except for Fe-peak elements. a: $[\mathrm{V} \mathrm{I} / \mathrm{Fe}]$ for stars possess $[\mathrm{Fe} / \mathrm{H}]>2.0$ is used for comparison. The red and blue dots correspond to RHB and BHB stars. 


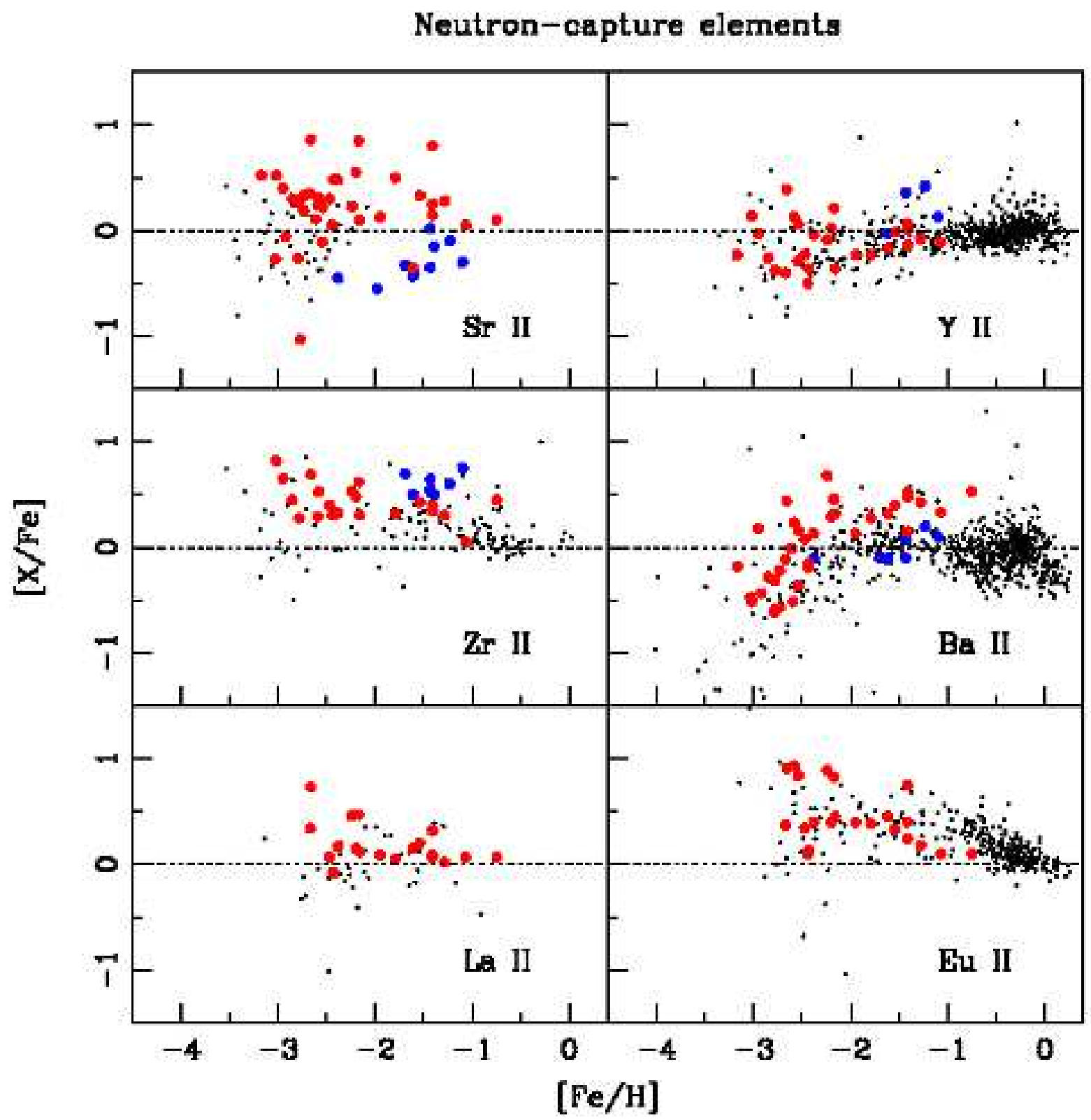

Fig. 25. - Same as Figure 23, except for $n$-capture elements. The red and blue dots correspond to RHB and BHB stars. 


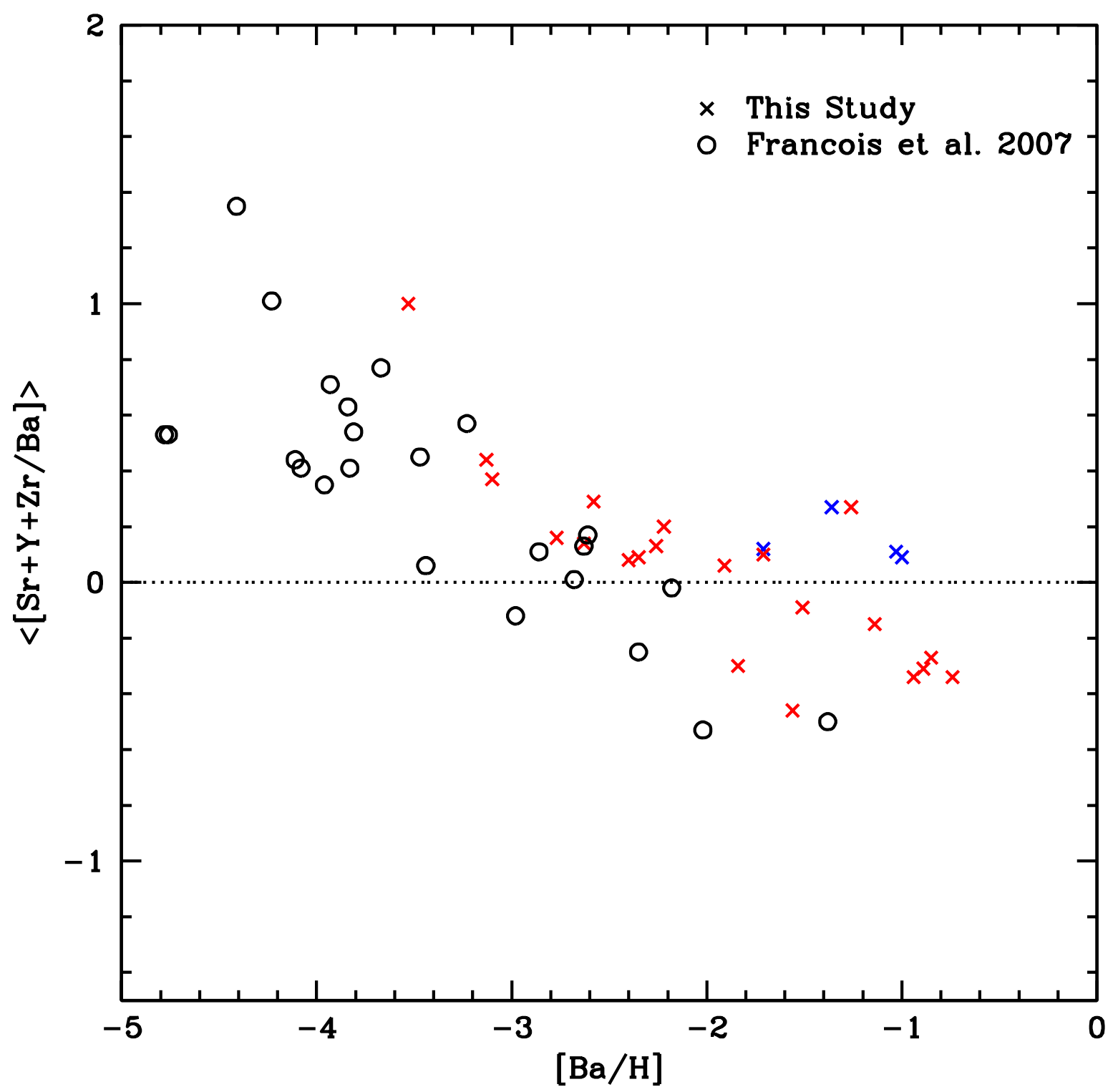

Fig. 26. - Mean abundance ratios of $[\mathrm{Sr}+\mathrm{Y}+\mathrm{Zr} / \mathrm{Ba}]$ vs $[\mathrm{Ba} / \mathrm{H}]$ (red crosses), with the additional data from François et al. (2007) (black open circles). 


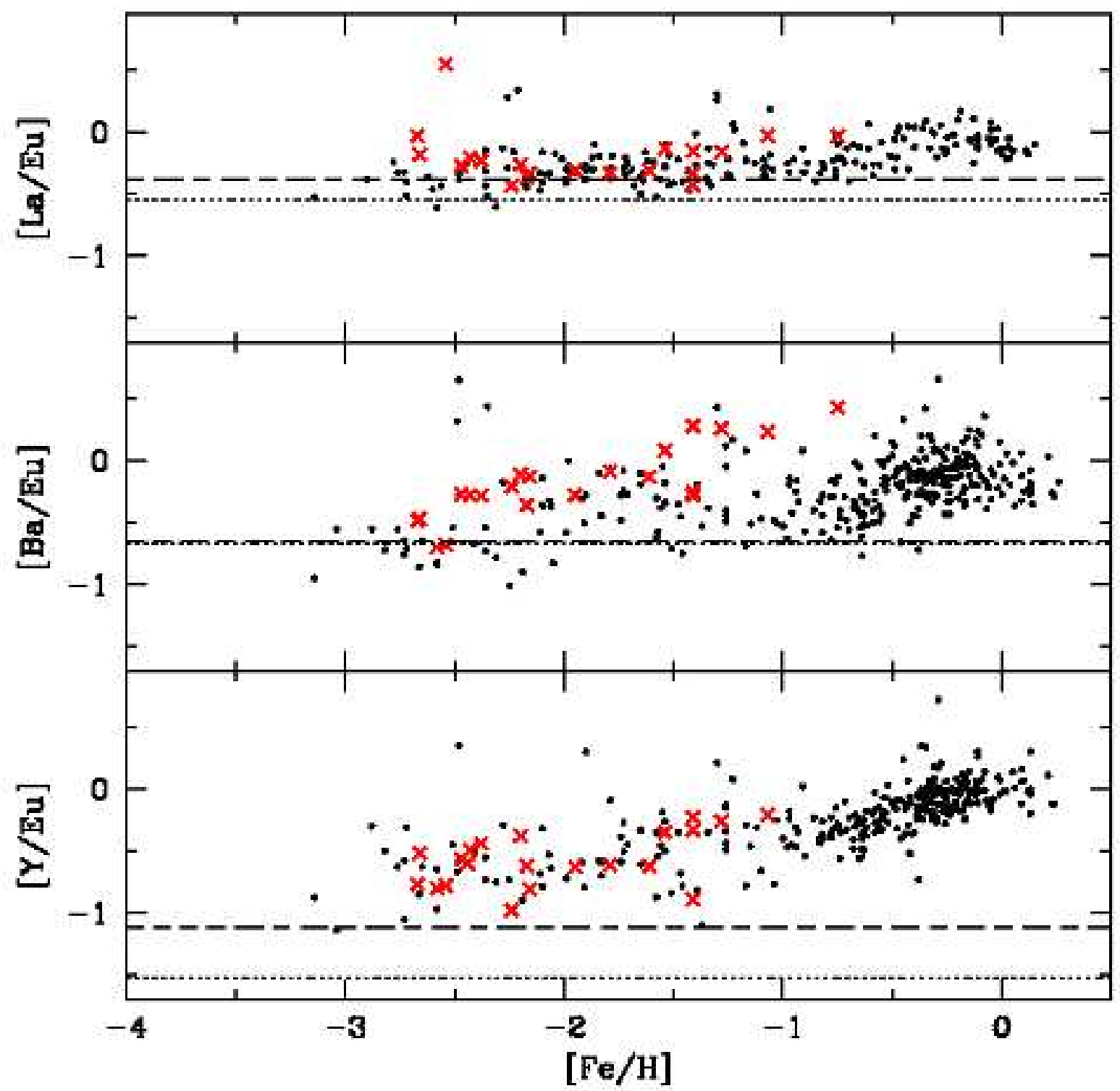

Fig. 27.- Comparison of light vs heavier $n$-capture elemental abundance ratios as a function of metallicity. These ratios are used to examine $s$ and $r$-process enrichment. The dashed and dotted lines represent the estimated pure $r$-process from solar system abundances of Arlandini et al. (1999) and Sneden et al. (2008), respectively. The red crosses correspond to our RHB stars. The black dots represent La, Ba, Y, Eu from Venn et al. (2004), La, Eu from Simmerer et al. (2004) and Woolf et al. (1995). 


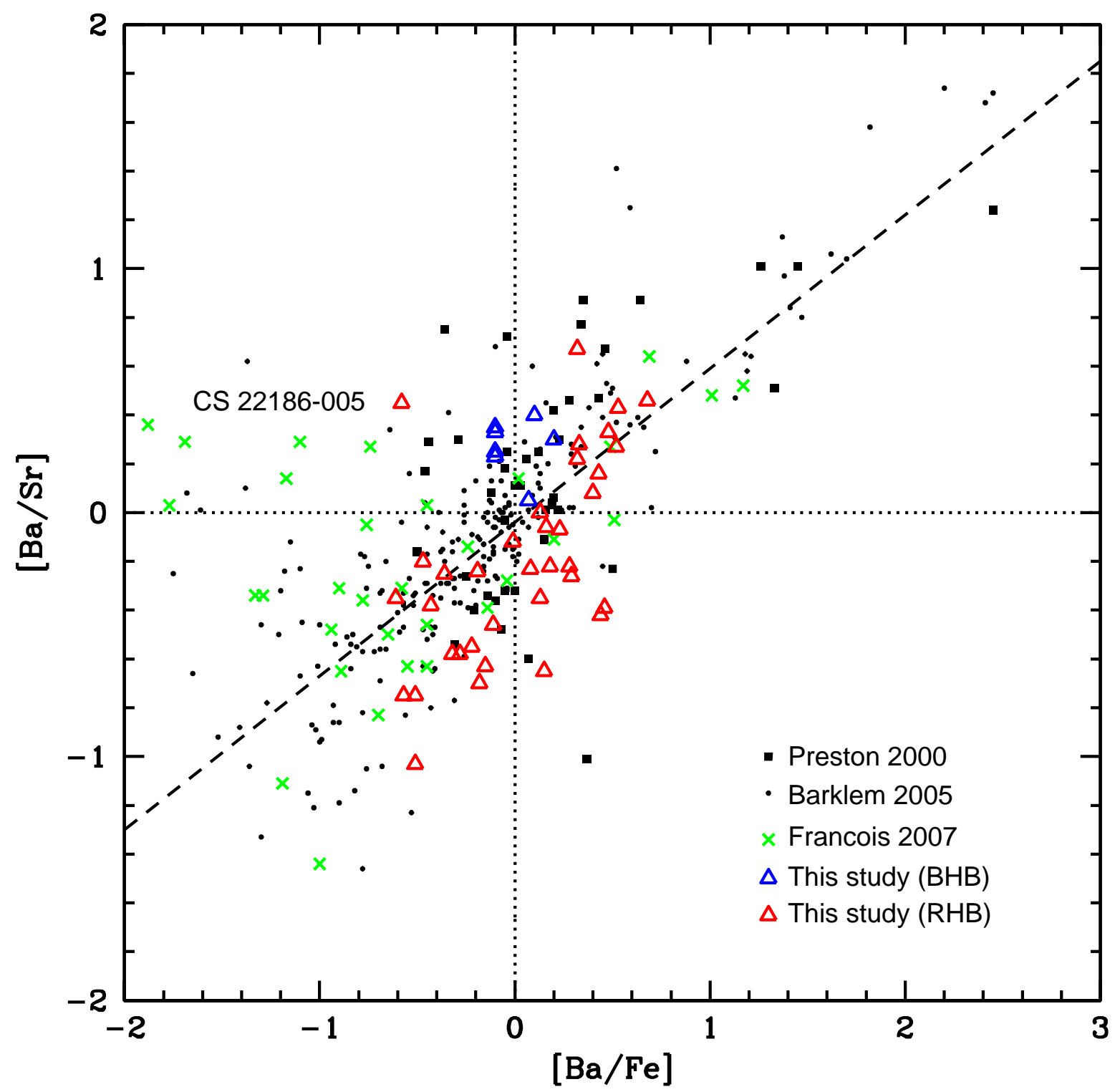

Fig. 28.- Abundance ratios of $[\mathrm{Ba} / \mathrm{Sr}]$ vs $[\mathrm{Ba} / \mathrm{Fe}]$. The long dashed line represent the linear correlation between $[\mathrm{Ba} / \mathrm{Sr}]$ and $[\mathrm{Ba} / \mathrm{Fe}]$ (see Sneden et al. 2008). Solid, black rectangulars and dots represent studies of Preston \& Sneden (2000) and Barklem et al. (2005), respectively. Study by François et al. (2007) is represented in green crosses. Our RHB and BHB stars are represented by red and blue open triangles. 
Table 1. Program stars.

\begin{tabular}{|c|c|c|c|c|c|c|c|c|c|c|c|}
\hline Star & $\begin{array}{l}\text { R.A.(J2000) } \\
\quad(\text { hr m s) }\end{array}$ & $\begin{array}{c}\text { Decl.(J2000) } \\
\left({ }^{\circ \prime \prime \prime}\right)\end{array}$ & $\begin{array}{c}B^{\mathrm{a}} \\
(\mathrm{mag})\end{array}$ & $\begin{array}{r}V^{\mathrm{a}, b} \\
(\mathrm{mag})\end{array}$ & $\begin{array}{c}J^{\mathrm{c}} \\
(\mathrm{mag})\end{array}$ & $\begin{array}{c}H^{\mathrm{c}} \\
(\mathrm{mag})\end{array}$ & $\begin{array}{c}K_{s}^{\mathrm{c}} \\
(\mathrm{mag})\end{array}$ & $\begin{array}{l}B-V \\
(\mathrm{mag})\end{array}$ & $\begin{array}{l}V-K \\
(\mathrm{mag})\end{array}$ & $\begin{array}{l}\mathrm{E}(B-V)^{\mathrm{d}} \\
\quad(\mathrm{mag})\end{array}$ & $\begin{array}{c}\mathrm{cE}(B-V) \\
\quad(\mathrm{mag})\end{array}$ \\
\hline \multicolumn{12}{|c|}{ RHB } \\
\hline HD 6229 & 010336.5 & +234606.4 & 9.31 & 8.60 & 7.088 & 6.646 & 6.575 & 0.71 & 2.025 & 0.034 & 0.021 \\
\hline HD 6461 & 010525.4 & -125412.1 & 8.4 & 7.65 & 6.149 & 5.676 & 5.587 & 0.75 & 2.063 & 0.025 & 0.013 \\
\hline HD 25532 & 040411.0 & +232427.1 & 8.85 & 8.24 & 6.688 & 6.327 & $\ldots$ & 0.61 & 1.057 & 0.191 & 0.162 \\
\hline HD 105546 & 120902.7 & +590105.1 & 9.4 & 8.61 & 7.152 & 6.756 & 6.674 & 0.79 & 0.980 & 0.022 & 0.010 \\
\hline HD 119516 & 134326.7 & +153431.1 & 9.52 & 9.13 & 7.771 & 7.431 & 7.366 & 0.39 & 1.764 & 0.031 & 0.018 \\
\hline $\mathrm{BD}+18^{\circ} 2890$ & 143213.5 & +172524.3 & 10.49 & 9.77 & 8.241 & 7.837 & 7.744 & 0.72 & 2.026 & 0.020 & 0.008 \\
\hline $\mathrm{BD}+11^{\circ} 2998$ & 163016.8 & +105951.7 & 9.70 & 9.07 & 7.619 & 7.271 & 7.185 & 0.63 & 1.885 & 0.057 & 0.041 \\
\hline $\mathrm{BD}+09^{\circ} 3223$ & 163335.6 & +0906 16.3 & 9.81 & 9.25 & 7.760 & 7.335 & 7.277 & 0.56 & 1.007 & 0.076 & 0.058 \\
\hline $\mathrm{BD}+17^{\circ} 3248$ & 172814.5 & +173035.8 & 9.99 & 9.37 & 7.876 & 7.391 & 7.338 & 0.62 & 0.956 & 0.059 & 0.043 \\
\hline HD 184266 & 193415.4 & -161900.2 & 8.16 & 7.57 & 6.252 & 5.913 & 5.830 & 0.59 & 1.740 & 0.142 & 0.118 \\
\hline HD 229274 & 202436.1 & +413002.6 & 9.63 & 9.06 & 7.622 & 7.288 & 7.213 & 0.57 & 1.847 & $\ldots$ & $\ldots$ \\
\hline CS 22882-001 & 002025.3 & -313904.0 & 15.22 & 14.82 & 13.677 & 13.362 & 13.317 & 0.40 & 1.503 & 0.018 & 0.006 \\
\hline CS 22190-007 & 035221.7 & -162430.0 & 14.66 & 14.20 & 13.059 & 12.706 & 12.656 & 0.46 & 1.544 & 0.031 & 0.018 \\
\hline CS 22186-005 & 041309.1 & -355038.7 & 13.33 & 12.96 & 11.902 & 11.625 & 11.581 & 0.37 & 1.379 & 0.012 & 0.001 \\
\hline CS 22191-029 & 044742.2 & -390726.0 & 14.46 & 14.05 & 12.947 & 12.646 & 12.614 & 0.41 & 1.436 & 0.019 & 0.007 \\
\hline CS 22883-037 & 142419.4 & +112925.0 & 15.28 & 14.73 & 13.733 & 13.425 & 13.378 & 0.55 & 1.352 & 0.028 & 0.015 \\
\hline CS 22878-121 & 164750.1 & +113912.0 & 14.53 & 13.99 & 12.620 & 12.288 & 12.169 & 0.54 & 1.821 & 0.043 & 0.029 \\
\hline CS 22891-184 & 192612.5 & -603409.0 & 14.33 & 13.83 & 12.574 & 12.274 & 12.187 & 0.50 & 1.643 & 0.070 & 0.053 \\
\hline CS 22896-110 & 193548.0 & -532617.0 & 14.09 & 13.56 & 12.180 & 11.791 & 11.780 & 0.53 & 1.780 & 0.060 & 0.044 \\
\hline CS 22940-077 & 204133.5 & -595036.0 & 14.66 & 14.13 & 12.679 & 12.300 & 12.220 & 0.53 & 1.910 & 0.070 & 0.053 \\
\hline CS 22955-174 & 204205.0 & -234912.7 & 14.88 & 14.38 & 13.179 & 12.843 & 12.770 & 0.50 & 1.610 & 0.049 & 0.034 \\
\hline CS 22940-070 & 204239.2 & -614041.0 & 15.35 & 14.87 & 13.686 & 13.368 & 13.312 & 0.48 & 1.558 & 0.056 & 0.040 \\
\hline CS 22879-103 & 204710.1 & -372652.6 & 14.79 & 14.30 & 13.095 & 12.747 & 12.661 & 0.49 & 1.639 & 0.044 & 0.030 \\
\hline CS 22879-097 & 204846.6 & -383049.4 & 14.68 & 14.22 & 13.031 & 12.684 & 12.617 & 0.46 & 1.603 & 0.048 & 0.033 \\
\hline CS 22940-121 & 205510.8 & -580054.0 & 14.71 & 14.16 & 12.738 & 12.339 & 12.267 & 0.55 & 1.893 & 0.053 & 0.038 \\
\hline CS 22898-043 & 211036.8 & -214451.8 & 14.49 & 14.06 & 12.909 & 12.674 & 12.650 & 0.43 & 1.410 & 0.050 & 0.035 \\
\hline CS 22937-072 & 211440.6 & -372451.8 & 14.55 & 14.02 & 12.646 & 12.301 & 12.221 & 0.53 & 1.799 & 0.040 & 0.026 \\
\hline CS 22948-006 & 213317.7 & -393942.8 & 15.56 & 15.07 & 13.774 & 13.405 & 13.334 & 0.49 & 1.736 & 0.030 & 0.017 \\
\hline CS 22944-039 & 214512.2 & -144122.0 & 14.85 & 14.30 & 12.976 & 12.616 & 12.500 & 0.55 & 1.800 & 0.049 & 0.034 \\
\hline CS 22951-077 & 215753.4 & -430806.0 & 14.11 & 13.61 & 12.258 & 11.944 & 11.845 & 0.50 & 1.765 & 0.016 & 0.004 \\
\hline CS 22881-039 & 220935.4 & -402551.2 & 15.52 & 15.12 & 13.915 & 13.746 & 13.646 & 0.40 & 1.474 & 0.014 & 0.003 \\
\hline CS 22886-043 & 222233.9 & -101411.0 & 15.18 & 14.72 & 13.564 & 13.247 & 13.178 & 0.46 & 1.542 & 0.047 & 0.032 \\
\hline CS 22875-029 & 222925.1 & -385747.5 & 14.08 & 13.68 & 12.584 & 12.298 & 12.267 & 0.40 & 1.413 & 0.013 & 0.002 \\
\hline CS 22888-047 & 232019.9 & -334546.9 & 15.01 & 14.61 & 13.460 & 13.194 & 13.127 & 0.40 & 1.483 & 0.019 & 0.007 \\
\hline CS 22941-027 & 233458.1 & -365205.7 & 14.40 & 14.05 & 13.060 & 12.721 & 12.747 & 0.35 & 1.303 & 0.016 & 0.004 \\
\hline CS 22945-056 & 235319.8 & -652941.0 & 14.485 & 14.09 & 12.984 & 12.692 & 12.616 & 0.40 & 1.474 & 0.020 & 0.008 \\
\hline \multicolumn{12}{|c|}{ BHB } \\
\hline HD 2857 & 003153.8 & -051542.9 & 10.12 & 9.95 & 9.481 & 9.354 & 9.323 & 0.17 & 0.627 & 0.041 & 0.027 \\
\hline HD 8376 & 012328.3 & +314712.3 & 9.72 & 9.59 & 9.248 & 9.163 & 9.130 & 0.13 & 0.460 & 0.051 & 0.036 \\
\hline HD 252940 & 061137.3 & +262730.1 & 9.4 & 9.096 & 8.440 & 8.371 & 8.302 & 0.30 & 0.794 & $\ldots$ & $\ldots$ \\
\hline HD 60778 & 073611.8 & -000815.6 & 9.19 & 9.12 & 8.746 & 8.662 & 8.666 & 0.07 & 0.454 & 0.104 & 0.084 \\
\hline HD 74721 & 084559.3 & +131548.7 & 8.76 & 8.71 & 8.521 & 8.525 & 8.522 & 0.05 & 0.188 & 0.031 & 0.018 \\
\hline HD 86986 & 100229.6 & +143325.2 & 8.11 & 8.01 & 7.610 & 7.499 & 7.499 & 0.10 & 0.511 & 0.031 & 0.018 \\
\hline
\end{tabular}


Table 1 -Continued

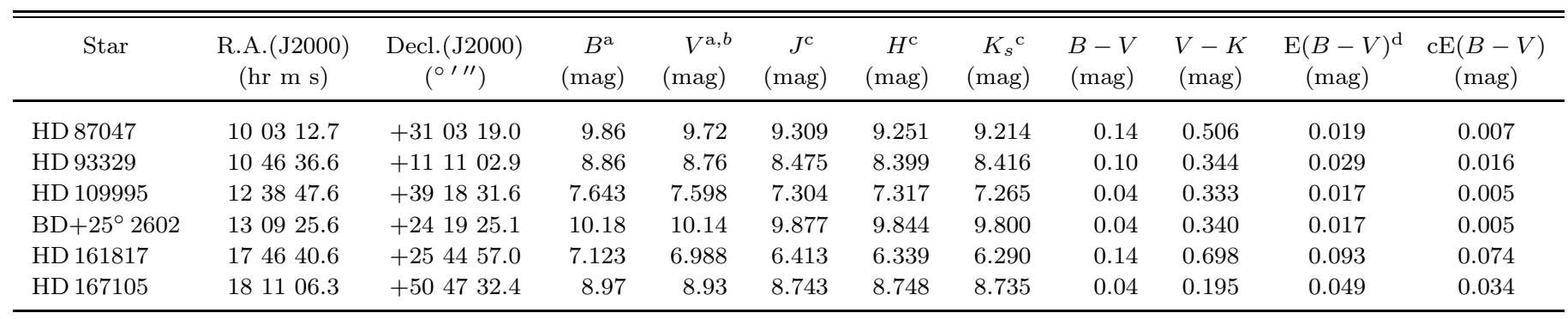

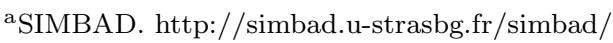

Beers et al. (1992).

c2MASS All-Sky Point Source Catalog(Skrutskie et al. 2006). http://tdc-www.harvard.edu/catalogs/tmpsc.html

${ }^{\mathrm{d}} \mathrm{Nasa} / \mathrm{IPAC}$ extragalactic database. 
Table 2. Observation Log.

\begin{tabular}{|c|c|c|c|c|c|c|c|}
\hline Star & UT Date & No. Integration & $\begin{array}{c}t_{\text {exp }} \\
(\mathrm{s})\end{array}$ & $\mathrm{S} / \mathrm{N}$ at $7000 \AA$ & $\mathrm{S} / \mathrm{N}$ at $5000 \AA$ & $\mathrm{S} / \mathrm{N}$ at $4000 \AA$ & Comments \\
\hline $\mathrm{BD}+09^{\circ} 3223$ & 30 Jun 2007 & 3 & 1800 & 223 & 230 & 95 & 1 \\
\hline $\mathrm{BD}+18^{\circ} 2890$ & 02 Jul 2007 & 3 & 1800 & 210 & 124 & 30 & 1 \\
\hline HD 180903 & 02 Jul 2007 & 3 & 1800 & 210 & 88 & 40 & 1,4 \\
\hline HD 229274 & 02 Jul 2007 & 3 & 1800 & 320 & 147 & 100 & 1 \\
\hline $\mathrm{BD}+17^{\circ} 3248$ & 04 Jul 2007 & 2 & 1800 & 280 & 108 & 66 & 1 \\
\hline HD 252940 & 20 Feb 2008 & 3 & 1800 & 188 & 135 & 63 & 1 \\
\hline HD 117880 & 21 Feb 2008 & 3 & 1800 & 196 & 96 & 86 & 1,3 \\
\hline HD 60778 & 21 Feb 2008 & 4 & $1 \times 1200,1 \times 1800$ & 200 & 125 & 64 & 1 \\
\hline HD 87112 & 21, 22 Feb 2008 & 5 & 1800 & 250 & 112 & 56 & 1,3 \\
\hline HD 25532 & 22 Feb 2008 & 3 & 1800 & 247 & 235 & 122 & 1 \\
\hline HD 109995 & 11 Apr 2009 & 4 & $3 \times 1200,1 \times 870$ & 370 & 124 & 72 & 2 \\
\hline HD 74721 & 11 Apr 2009 & 4 & $1 \times 1200,3 \times 1800$ & 200 & 156 & 86 & 2 \\
\hline HD 161817 & 11 Apr 2009 & 4 & 1200 & 430 & 270 & 73 & 2 \\
\hline HD 167105 & 11, 13 Apr 2009 & 4 & $3 \times 1800,1 \times 2400$ & 260 & 162 & 67 & 2 \\
\hline HD 93329 & 13 Apr 2009 & 5 & $1 \times 1000,3 \times 2400$ & 290 & 109 & 163 & 2 \\
\hline HD 87047 & 14 Apr 2009 & 3 & 2400 & 150 & 96 & 67 & 2 \\
\hline HD 105546 & 14 Apr 2009 & 4 & $3 \times 1800,1 \times 1400$ & 250 & 190 & 70 & 2 \\
\hline HD 8376 & 06 Oct 2009 & 3 & 1800 & 200 & 105 & 67 & 2 \\
\hline HD 2857 & 08,09 Oct 2009 & 4 & $3 \times 1800,1 \times 1000$ & 170 & 100 & 34 & 2 \\
\hline HD 6229 & 09 Oct 2009 & 3 & 1200 & 200 & 166 & 74 & 2 \\
\hline
\end{tabular}

${ }^{1}$ The echelle grating was blazed to obtain more red portion of the spectrum. See text for explanation.

${ }^{2}$ The echelle grating was blazed to obtain optima red and blue portion of the spectrum.

${ }^{3}$ Initial analysis was performed. Stellar parameters cannot be obtained due to the lack of measurable Fe I or Fe II lines. Excluded from this study.

${ }^{4} \mathrm{RR}$ Lyr, excluded from this study. 
Table 3. Equivalent width measurements of program stars.

\begin{tabular}{cccccc}
\hline \hline $\begin{array}{c}\text { Wavelength } \\
(\AA)\end{array}$ & Species & $\begin{array}{c}\text { E.P. } \\
(\mathrm{eV})\end{array}$ & $\log g f$ & Ref. & $\begin{array}{c}\text { EW } \\
(\mathrm{m} \AA)\end{array}$ \\
\hline \multicolumn{7}{c}{ HD 6229} \\
\hline 5682.63 & Na I & 2.102 & -0.71 & 1 & 49 \\
5688.19 & Na I & 2.104 & -0.46 & 1 & $\ldots$ \\
5339.93 & Fe I & 3.266 & -0.72 & 1 & 101 \\
5341.02 & Fe I & 1.608 & -1.95 & 1 & 141 \\
. &. &. &. &. &. \\
. &. &. &. &. &. \\
. &. &. &. &. &. \\
\hline
\end{tabular}

Note. - Table 3 is published in its entirety in the electronic edition of the Astronomical Journal. A portion is shown here for guidance regarding its form and content. 
Table 4. Input stellar atmosphere parameters and derived Fe metallicities.

\begin{tabular}{|c|c|c|c|c|c|c|c|c|c|c|}
\hline Star & $\begin{array}{l}T_{\text {eff }} \\
(\mathrm{K})\end{array}$ & $\begin{array}{l}\log g \\
(\operatorname{dex})\end{array}$ & {$[\mathrm{M} / \mathrm{H}]^{\mathrm{a}}$} & $\begin{array}{c}v_{t} \\
\left(\mathrm{~km} \mathrm{~s}^{-1}\right)\end{array}$ & {$[\mathrm{Fe} \mathrm{I} / \mathrm{H}]$} & $\sigma$ & $\mathrm{N}$ & {$[\mathrm{Fe} \mathrm{II} / \mathrm{H}]$} & $\sigma$ & $\mathrm{N}$ \\
\hline \multicolumn{11}{|c|}{ RHB } \\
\hline HD 6229 & 5200 & 2.50 & -1.07 & 1.60 & -1.07 & 0.13 & 98 & -1.06 & 0.13 & 20 \\
\hline HD 6461 & 5200 & 2.90 & -0.75 & 1.40 & -0.75 & 0.12 & 94 & -0.74 & 0.10 & 13 \\
\hline HD 25532 & 5450 & 2.00 & -1.41 & 2.10 & -1.41 & 0.06 & 44 & -1.42 & 0.09 & 8 \\
\hline HD 105546 & 5200 & 2.30 & -1.54 & 1.80 & -1.54 & 0.08 & 65 & -1.54 & 0.06 & 20 \\
\hline HD 119516 & 5400 & 1.50 & -2.16 & 2.20 & -2.16 & 0.06 & 49 & -2.16 & 0.05 & 15 \\
\hline $\mathrm{BD}+18^{\circ} 2890$ & 5000 & 2.40 & -1.61 & 1.40 & -1.61 & 0.07 & 51 & -1.61 & 0.09 & 8 \\
\hline $\mathrm{BD}+11^{\circ} 2998$ & 5450 & 2.30 & -1.28 & 1.90 & -1.28 & 0.08 & 59 & -1.29 & 0.06 & 10 \\
\hline $\mathrm{BD}+09^{\circ} 3223$ & 5100 & 1.30 & -2.47 & 1.90 & -2.47 & 0.05 & 48 & -2.46 & 0.06 & 11 \\
\hline $\mathrm{BD}+17^{\circ} 3248$ & 5100 & 1.70 & -2.24 & 1.80 & -2.24 & 0.06 & 38 & -2.23 & 0.07 & 13 \\
\hline HD 184266 & 5700 & 1.70 & -1.79 & 2.70 & -1.79 & 0.06 & 32 & -1.78 & 0.05 & 8 \\
\hline HD 229274 & 5500 & 2.30 & -1.41 & 2.00 & -1.41 & 0.08 & 44 & -1.42 & 0.08 & 12 \\
\hline CS 22882-001 & 5950 & 2.00 & -2.50 & 3.05 & -2.54 & 0.10 & 55 & -2.54 & 0.07 & 14 \\
\hline CS 22190-007 & 5600 & 1.90 & -2.50 & 1.90 & -2.67 & 0.09 & 93 & -2.67 & 0.07 & 15 \\
\hline CS 22186-005 & 6200 & 2.45 & -2.50 & 3.20 & -2.77 & 0.07 & 13 & -2.78 & 0.08 & 6 \\
\hline CS 22191-029 & 6000 & 2.10 & -2.50 & 2.90 & -2.73 & 0.09 & 53 & -2.72 & 0.06 & 10 \\
\hline CS $22883-037$ & 5900 & 1.65 & -1.95 & 2.80 & -1.95 & 0.11 & 73 & -1.94 & 0.10 & 17 \\
\hline CS $22878-121$ & 5450 & 1.75 & -2.38 & 1.90 & -2.38 & 0.12 & 110 & -2.37 & 0.07 & 24 \\
\hline CS $22891-184$ & 5600 & 1.70 & -2.50 & 2.05 & -2.61 & 0.07 & 86 & -2.61 & 0.07 & 16 \\
\hline CS 22896-110 & 5400 & 1.45 & -2.50 & 2.05 & -2.78 & 0.09 & 78 & -2.78 & 0.07 & 16 \\
\hline CS $22940-077$ & 5300 & 1.45 & -2.50 & 1.90 & -3.02 & 0.08 & 70 & -3.02 & 0.09 & 15 \\
\hline CS 22955-174 & 5350 & 1.35 & -2.50 & 2.20 & -3.17 & 0.09 & 45 & -3.17 & 0.08 & 7 \\
\hline CS $22940-070$ & 6300 & 2.40 & -1.41 & 3.20 & -1.41 & 0.07 & 24 & -1.42 & 0.06 & 7 \\
\hline CS 22879-103 & 5700 & 1.60 & -2.20 & 3.00 & -2.20 & 0.08 & 94 & -2.20 & 0.06 & 16 \\
\hline CS 22879-097 & 5650 & 1.95 & -2.50 & 2.20 & -2.59 & 0.10 & 76 & -2.58 & 0.10 & 14 \\
\hline CS $22940-121$ & 5350 & 1.60 & -2.50 & 2.10 & -2.95 & 0.09 & 73 & -2.94 & 0.12 & 14 \\
\hline CS $22898-043$ & 5900 & 2.00 & -2.50 & 3.40 & -3.03 & 0.05 & 12 & -3.03 & 0.08 & 2 \\
\hline CS 22937-072 & 5300 & 1.50 & -2.50 & 1.80 & -2.85 & 0.09 & 86 & -2.85 & 0.06 & 16 \\
\hline CS 22948-006 & 5400 & 1.40 & -2.50 & 2.15 & -2.79 & 0.09 & 83 & -2.79 & 0.09 & 13 \\
\hline CS 22944-039 & 5350 & 1.20 & -2.43 & 2.20 & -2.43 & 0.10 & 99 & -2.44 & 0.09 & 16 \\
\hline CS 22951-077 & 5350 & 1.55 & -2.44 & 2.00 & -2.44 & 0.09 & 97 & -2.43 & 0.09 & 13 \\
\hline CS 22881-039 & 6100 & 1.85 & -2.50 & 2.70 & -2.73 & 0.08 & 37 & -2.72 & 0.12 & 7 \\
\hline CS $22886-043$ & 6000 & 1.85 & -2.17 & 3.05 & -2.17 & 0.11 & 52 & -2.17 & 0.10 & 21 \\
\hline CS $22875-029$ & 6000 & 2.05 & -2.50 & 3.00 & -2.66 & 0.09 & 62 & -2.66 & 0.08 & 12 \\
\hline CS 22888-047 & 5850 & 1.70 & -2.50 & 3.20 & -2.58 & 0.08 & 58 & -2.57 & 0.06 & 11 \\
\hline CS $22941-027$ & 6200 & 2.20 & -2.50 & 3.30 & -2.54 & 0.07 & 36 & -2.53 & 0.09 & 10 \\
\hline CS 22945-056 & 5850 & 1.50 & -2.50 & 3.00 & -2.92 & 0.07 & 33 & -2.92 & 0.08 & 7 \\
\hline \multicolumn{11}{|c|}{ ВHB } \\
\hline HD 2857 & 8100 & 3.60 & -1.39 & 3.70 & -1.39 & 0.13 & 12 & -1.38 & 0.14 & 14 \\
\hline HD 8376 & 8600 & 3.70 & -2.39 & 1.00 & -2.39 & 0.11 & 9 & -2.38 & 0.11 & 6 \\
\hline HD 252940 & 7650 & 2.70 & -1.69 & 3.10 & -1.69 & 0.07 & 11 & -1.68 & 0.07 & 10 \\
\hline HD 60778 & 8100 & 2.75 & -1.43 & 2.20 & -1.43 & 0.06 & 20 & -1.43 & 0.03 & 11 \\
\hline HD 74721 & 9000 & 3.40 & -1.23 & 1.40 & -1.23 & 0.05 & 13 & -1.21 & 0.06 & 13 \\
\hline HD 86986 & 8200 & 3.20 & -1.61 & 2.30 & -1.61 & 0.09 & 34 & -1.59 & 0.07 & 23 \\
\hline
\end{tabular}


Table 4-Continued

\begin{tabular}{lccccccccccr}
\hline \hline \multicolumn{1}{c}{ Star } & $\begin{array}{c}T_{\text {eff }} \\
(\mathrm{K})\end{array}$ & $\begin{array}{c}\log g \\
(\mathrm{dex})\end{array}$ & {$[\mathrm{M} / \mathrm{H}]^{\mathrm{a}}$} & $\begin{array}{c}v_{t} \\
\left(\mathrm{~km} \mathrm{~s}^{-1}\right)\end{array}$ & {$[\mathrm{Fe} \mathrm{I} / \mathrm{H}]$} & $\sigma$ & $\mathrm{N}$ & {$[\mathrm{Fe} \mathrm{II} / \mathrm{H}]$} & $\sigma$ & $\mathrm{N}$ \\
\hline HD 87047 & 7700 & 2.30 & -2.38 & 1.30 & -2.38 & 0.03 & 4 & -2.37 & 0.11 & 7 \\
HD 93329 & 8700 & 3.40 & -1.10 & 2.80 & -1.10 & 0.07 & 35 & -1.11 & 0.07 & 27 \\
HD 109995 & 8600 & 3.00 & -1.60 & 2.00 & -1.60 & 0.05 & 7 & -1.59 & 0.07 & 18 \\
BD +25 2602 & 8400 & 2.80 & -1.98 & 2.30 & -1.98 & 0.07 & 5 & -1.98 & 0.11 & 8 \\
HD 161817 & 7800 & 3.00 & -1.43 & 3.20 & -1.43 & 0.09 & 57 & -1.45 & 0.07 & 28 \\
HD 167105 & 9000 & 3.10 & -1.55 & 2.00 & -1.55 & 0.03 & 3 & -1.54 & 0.07 & 18 \\
\hline
\end{tabular}

anput model metallicity. 
Table 5. Abundance ratios from $\mathrm{Mg}, \mathrm{Si}$ and $\mathrm{Ca}$

\begin{tabular}{|c|c|c|c|c|c|c|c|c|c|c|c|c|c|c|c|c|c|c|}
\hline Star & {$[\mathrm{Na} \mathrm{I} / \mathrm{Fe}]$} & $\sigma$ & $\mathrm{N}$ & {$[\mathrm{Mg} \mathrm{I} / \mathrm{Fe}]$} & $\sigma$ & $\mathrm{N}$ & {$[\mathrm{Si} \mathrm{I} / \mathrm{Fe}]$} & $\sigma$ & $\mathrm{N}$ & {$[\mathrm{Si} \mathrm{II} / \mathrm{Fe}]$} & $\sigma$ & $\mathrm{N}$ & {$[\mathrm{Ca} \mathrm{I} / \mathrm{Fe}]$} & $\sigma$ & $\mathrm{N}$ & {$[\mathrm{Ca}$ II $/ \mathrm{Fe}]$} & $\sigma$ & $\mathrm{N}$ \\
\hline \multicolumn{19}{|c|}{ RHB } \\
\hline HD 6229 & 0.03 & 0.06 & 5 & 0.36 & 0.04 & 3 & 0.28 & 0.06 & 5 & 0.32 & 0.03 & 2 & 0.15 & 0.11 & 12 & $\ldots$ & $\ldots$ & .. \\
\hline HD 6461 & -0.02 & 0.10 & 3 & 0.35 & 0.15 & 2 & 0.29 & 0.02 & 6 & 0.47 & 0.16 & 2 & 0.17 & 0.09 & 13 & $\ldots$ & $\cdots$ & ... \\
\hline HD 25532 & 0.64 & 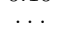 & 1 & 0.56 & & 1 & 0.53 & 0.07 & 5 & 0.54 & 0.18 & 2 & 0.29 & 0.05 & 4 & $\ldots$ & $\ldots$ & $\ldots$ \\
\hline HD 105546 & 0.17 & $\ldots$ & 1 & 0.50 & 0.08 & 3 & 0.40 & 0.10 & 6 & 0.61 & 0.20 & 3 & 0.42 & 0.09 & 12 & $\cdots$ & $\cdots$ & $\cdots$ \\
\hline HD 119516 & 0.54 & $\ldots$ & 1 & 0.28 & $\ldots$ & 1 & 0.40 & & 1 & 0.48 & 0.17 & 2 & 0.26 & 0.07 & 7 & $\ldots$ & $\cdots$ & $\cdots$ \\
\hline $\mathrm{BD}+18^{\circ} 2890$ & -0.04 & 0.02 & 4 & -0.06 & $\ldots$ & 1 & 0.41 & 0.08 & 6 & 0.74 & & 1 & 0.35 & 0.07 & 12 & $\cdots$ & $\cdots$ & $\cdots$ \\
\hline $\mathrm{BD}+11^{\circ} 2998$ & 0.24 & $\ldots$ & 1 & 0.56 & 0.12 & 2 & 0.41 & 0.07 & 5 & 0.52 & 0.07 & 3 & 0.29 & 0.09 & 7 & $\ldots$ & $\ldots$ & $\ldots$ \\
\hline $\mathrm{BD}+09^{\circ} 3223$ & & $\ldots$ & $\ldots$ & 0.27 & $\ldots$ & 1 & 0.73 & & 1 & 0.86 & 0.16 & 2 & 0.50 & 0.06 & 11 & $\ldots$ & & $\ldots$ \\
\hline $\mathrm{BD}+17^{\circ} 3248$ & 0.59 & $\ldots$ & 1 & 0.43 & 0.26 & 2 & 0.45 & & 1 & 0.84 & & 1 & 0.38 & 0.05 & 7 & $\ldots$ & & \\
\hline HD 184266 & 0.98 & $\ldots$ & 1 & 0.50 & 0.03 & 2 & 0.56 & 0.02 & 2 & 0.44 & $\ldots$ & 1 & 0.38 & 0.09 & 7 & $\ldots$ & $\ldots$ & $\ldots$ \\
\hline HD 229274 & 0.39 & 0.02 & 2 & 0.32 & 0.05 & 3 & 0.40 & 0.08 & 7 & 0.38 & 0.17 & 2 & 0.24 & 0.07 & 7 & $\ldots$ & $\ldots$ & $\ldots$ \\
\hline CS 22882-001 & & & & 0.37 & 0.01 & 2 & 0.00 & . & 1 & 0.48 & 0.06 & 2 & 0.40 & 0.09 & 6 & $\ldots$ & $\ldots$ & $\ldots$ \\
\hline CS $22190-007$ & 0.80 & 0.10 & 2 & 0.53 & 0.13 & 3 & 0.65 & $\ldots$ & 1 & 0.66 & & 1 & 0.35 & 0.08 & 10 & $\cdots$ & $\cdots$ & $\ldots$ \\
\hline CS $22186-005$ & -0.04 & $\ldots$ & 1 & 0.38 & 0.06 & 2 & $-0.11^{\mathrm{a}}$ & $\ldots$ & 1 & $0.36^{\mathrm{a}}$ & $\ldots$ & 1 & 0.19 & $\ldots$ & 1 & $\ldots$ & $\ldots$ & $\ldots$ \\
\hline CS 22191-029 & 0.13 & 0.02 & 2 & 0.57 & 0.15 & 4 & $0.15^{\mathrm{a}}$ & $\ldots$ & 1 & 0.55 & $\ldots$ & 1 & 0.39 & 0.10 & 9 & $\ldots$ & $\ldots$ & $\ldots$ \\
\hline CS 22883-037 & 0.81 & $\ldots$ & 1 & 0.04 & $\ldots$ & 1 & -0.14 & & 1 & 0.60 & 0.20 & 2 & 0.40 & 0.08 & 8 & $\cdots$ & $\ldots$ & $\ldots$ \\
\hline CS $22878-121$ & 0.47 & 0.26 & 2 & 0.41 & 0.08 & 5 & 0.69 & $\ldots$ & 1 & 0.30 & 0.14 & 2 & 0.38 & 0.08 & 13 & $\ldots$ & $\ldots$ & $\ldots$ \\
\hline CS $22891-184$ & & & $\ldots$ & 0.40 & 0.13 & 5 & 0.37 & $\ldots$ & 1 & 0.45 & 0.08 & 2 & 0.32 & 0.05 & 9 & $\ldots$ & $\ldots$ & $\ldots$ \\
\hline CS $22896-110$ & 0.87 & 0.02 & 2 & 0.59 & 0.10 & 3 & 0.61 & $\ldots$ & 1 & 0.53 & 0.12 & 3 & 0.41 & 0.06 & 8 & $\ldots$ & $\ldots$ & $\ldots$ \\
\hline CS $22940-077$ & 0.67 & 0.00 & 2 & 0.61 & 0.07 & 4 & 0.33 & $\ldots$ & 1 & 0.62 & $\ldots$ & 1 & 0.49 & 0.08 & 9 & $\cdots$ & $\cdots$ & $\ldots$ \\
\hline CS $22955-174$ & $\ldots$ & $\ldots$ & $\ldots$ & 0.74 & 0.04 & 4 & 0.30 & $\ldots$ & 1 & 1.34 & & 1 & 0.58 & 0.09 & 6 & $\ldots$ & $\ldots$ & $\ldots$ \\
\hline CS $22940-070$ & $\ldots$ & $\ldots$ & $\cdots$ & 0.44 & $\ldots$ & 1 & 0.66 & 0.11 & 4 & 0.33 & 0.05 & 2 & 0.19 & 0.06 & 6 & $\cdots$ & $\ldots$ & $\ldots$ \\
\hline CS $22879-103$ & $\ldots$ & $\ldots$ & $\ldots$ & 0.50 & 0.09 & 3 & 0.38 & & 1 & 0.63 & 0.05 & 3 & 0.44 & 0.06 & 12 & $\ldots$ & $\ldots$ & $\ldots$ \\
\hline CS $22879-097$ & $\ldots$ & $\ldots$ & $\ldots$ & 0.79 & 0.03 & 2 & 0.22 & $\ldots$ & 1 & 0.88 & 0.20 & 2 & 0.45 & 0.10 & 9 & $\cdots$ & $\ldots$ & $\ldots$ \\
\hline CS $22940-121$ & $\ldots$ & $\ldots$ & $\ldots$ & 0.61 & 0.04 & 4 & 0.85 & $\ldots$ & 1 & 0.83 & $\ldots$ & 1 & 0.45 & 0.07 & 4 & $\ldots$ & $\ldots$ & $\ldots$ \\
\hline CS $22898-043$ & $\ldots$ & $\ldots$ & $\ldots$ & 0.52 & 0.02 & 3 & -0.14 & & 1 & $\cdots$ & & ... & 0.41 & 0.03 & 3 & $\cdots$ & $\ldots$ & \\
\hline CS $22937-072$ & 0.49 & 0.08 & 2 & 0.70 & 0.10 & 3 & 0.50 & $\ldots$ & 1 & 1.12 & 0.02 & 2 & 0.55 & 0.07 & 8 & $\ldots$ & $\ldots$ & $\ldots$ \\
\hline CS $22948-006$ & 0.39 & 0.13 & 2 & 0.57 & 0.06 & 2 & 0.41 & $\ldots$ & 1 & 0.90 & 0.16 & 2 & 0.59 & 0.09 & 12 & $\ldots$ & $\ldots$ & $\ldots$ \\
\hline CS 22944-039 & 0.56 & 0.15 & 2 & 0.41 & 0.02 & 2 & 0.55 & $\ldots$ & 1 & 0.52 & 0.15 & 2 & 0.40 & 0.07 & 10 & $\ldots$ & $\ldots$ & $\ldots$ \\
\hline CS 22951-077 & 0.26 & 0.04 & 2 & 0.45 & 0.09 & 4 & 0.51 & $\ldots$ & 1 & 0.44 & 0.01 & 2 & 0.39 & 0.07 & 15 & $\ldots$ & $\ldots$ & $\ldots$ \\
\hline CS 22881-039 & 0.12 & 0.05 & 2 & 0.70 & 0.01 & 2 & $0.08^{\mathrm{a}}$ & $\cdots$ & 1 & $0.27^{\mathrm{a}}$ & & 1 & 0.52 & 0.09 & 4 & $\cdots$ & $\cdots$ & $\cdots$ \\
\hline CS $22886-043$ & 0.65 & 0.18 & 2 & 0.45 & 0.08 & 3 & $0.40^{\mathrm{a}}$ & $\ldots$ & 1 & 0.29 & $\ldots$ & 1 & 0.35 & 0.09 & 6 & $\ldots$ & $\ldots$ & $\ldots$ \\
\hline CS $22875-029$ & 0.41 & $\ldots$ & 1 & 0.59 & $\ldots$ & 1 & $0.17^{\mathrm{a}}$ & & 1 & $0.53^{\mathrm{a}}$ & 0.10 & 3 & 0.45 & 0.04 & 6 & $\ldots$ & $\ldots$ & \\
\hline CS $22888-047$ & -0.16 & $\ldots$ & 1 & 0.27 & 0.01 & 2 & 0.06 & $\ldots$ & 1 & 0.61 & $\ldots$ & 1 & 0.34 & 0.09 & 7 & $\ldots$ & $\ldots$ & $\ldots$ \\
\hline CS $22941-027$ & -0.14 & 0.10 & 2 & 0.32 & 0.10 & 2 & $0.16^{\mathrm{a}}$ & $\ldots$ & 1 & $0.33^{\mathrm{a}}$ & $\ldots$ & 1 & 0.22 & 0.11 & 4 & $\ldots$ & $\ldots$ & $\ldots$ \\
\hline CS $22945-056$ & 0.27 & $\ldots$ & 1 & 0.78 & 0.18 & 2 & 0.12 & $\ldots$ & 1 & 0.86 & $\ldots$ & 1 & 0.41 & 0.11 & 3 & $\cdots$ & $\cdots$ & $\ldots$ \\
\hline \multicolumn{19}{|c|}{ BHB } \\
\hline HD 2857 & $\ldots$ & $\ldots$ & $\ldots$ & 0.31 & 0.14 & 2 & $-0.22^{\mathrm{a}}$ & & 1 & $0.13^{\mathrm{a}}$ & 0.08 & 2 & 0.33 & $\ldots$ & 1 & 0.30 & & 1 \\
\hline HD 8376 & $\ldots$ & $\ldots$ & $\ldots$ & 0.05 & 0.05 & 2 & $-0.04^{\mathrm{a}}$ & $\ldots$ & 1 & $0.34^{\mathrm{a}}$ & & 1 & -0.19 & 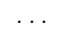 & 1 & 0.40 & & 1 \\
\hline HD 252940 & $\ldots$ & $\ldots$ & $\ldots$ & 0.36 & 0.01 & 2 & $-0.08^{\mathrm{a}}$ & $\ldots$ & 1 & $0.16^{\mathrm{a}}$ & & 1 & 0.40 & 0.07 & 4 & 0.35 & $\ldots$ & 1 \\
\hline HD 60778 & $\ldots$ & $\ldots$ & $\ldots$ & 0.38 & 0.02 & 2 & $-0.11^{\mathrm{a}}$ & $\ldots$ & 1 & $0.19^{\mathrm{a}}$ & 0.22 & 2 & 0.21 & 0.08 & 5 & 0.12 & $\ldots$ & 1 \\
\hline HD 74721 & $-0.41^{\mathrm{a}}$ & $\ldots$ & 1 & 0.35 & 0.02 & 2 & $0.07^{\mathrm{a}}$ & $\ldots$ & 1 & $0.45^{\mathrm{a}}$ & 0.21 & 2 & -0.11 & $\ldots$ & 1 & 0.00 & $\ldots$ & 1 \\
\hline HD 86986 & $\ldots$ & $\ldots$ & $\ldots$ & 0.31 & 0.02 & 2 & $-0.10^{\mathrm{a}}$ & & 1 & $0.18^{\mathrm{a}}$ & 0.18 & 3 & 0.14 & 0.07 & 2 & 0.23 & $\ldots$ & 1 \\
\hline HD 87047 & & $\ldots$ & $\ldots$ & 0.65 & $\ldots$ & 1 & $0.04^{\mathrm{a}}$ & $\ldots$ & 1 & $0.22^{\mathrm{a}}$ & & 1 & 0.15 & $\ldots$ & 1 & 0.15 & $\ldots$ & 1 \\
\hline HD 93329 & $-0.49^{\mathrm{a}}$ & $\ldots$ & 1 & 0.24 & $\ldots$ & 1 & $-0.05^{\mathrm{a}}$ & $\ldots$ & 1 & $0.02^{\mathrm{a}}$ & 0.22 & 3 & -0.12 & $\ldots$ & 1 & 0.16 & $\ldots$ & 1 \\
\hline HD 109995 & & $\ldots$ & & 0.47 & $\ldots$ & 1 & $0.03^{\mathrm{a}}$ & $\ldots$ & 1 & $0.17^{\mathrm{a}}$ & 0.18 & 3 & 0.04 & $\ldots$ & 1 & 0.08 & $\ldots$ & 1 \\
\hline
\end{tabular}


Table 5-Continued

\begin{tabular}{|c|c|c|c|c|c|c|c|c|c|c|c|c|c|c|c|c|c|c|}
\hline Star & {$[\mathrm{Na} \mathrm{I} / \mathrm{Fe}]$} & $\sigma$ & $\mathrm{N}$ & {$[\mathrm{Mg} \mathrm{I} / \mathrm{Fe}]$} & $\sigma$ & $\mathrm{N}$ & {$[\mathrm{Si} \mathrm{I} / \mathrm{Fe}]$} & $\sigma$ & $\mathrm{N}$ & {$[\mathrm{Si} \mathrm{II} / \mathrm{Fe}]$} & $\sigma$ & $\mathrm{N}$ & {$[\mathrm{Ca} \mathrm{I} / \mathrm{Fe}]$} & $\sigma$ & $\mathrm{N}$ & {$[\mathrm{Ca} \mathrm{II} / \mathrm{Fe}]$} & $\sigma$ & $\mathrm{N}$ \\
\hline $\mathrm{BD}+25^{\circ} 2602$ & $\ldots$ & $\ldots$ & $\ldots$ & 0.50 & 0.05 & 2 & $0.15^{\mathrm{a}}$ & $\ldots$ & 1 & $0.41^{\mathrm{a}}$ & 0.17 & 2 & -0.03 & & 1 & 0.11 & $\ldots$ & 1 \\
\hline HD 161817 & $\cdots$ & $\ldots$ & $\ldots$ & 0.26 & 0.00 & 2 & $-0.09^{\mathrm{a}}$ & $\ldots$ & 1 & $0.06^{\mathrm{a}}$ & 0.15 & 3 & 0.24 & 0.05 & 8 & 0.32 & $\ldots$ & 1 \\
\hline HD 167105 & $\ldots$ & $\ldots$ & & 0.39 & 0.06 & 2 & $0.05^{\mathrm{a}}$ & $\ldots$ & 1 & $0.16^{\mathrm{a}}$ & 0.20 & 3 & -0.21 & $\ldots$ & 1 & -0.12 & & 1 \\
\hline
\end{tabular}

${ }^{\mathrm{a}}$ NLTE correction. 
Table 6. Abundance ratios of $\mathrm{Al}, \mathrm{Ti}, \mathrm{Sc}$ and $\mathrm{Cr}$

\begin{tabular}{|c|c|c|c|c|c|c|c|c|c|c|c|c|c|c|c|c|c|c|}
\hline Star & {$[\mathrm{Al} \mathrm{I} / \mathrm{Fe}]$} & $\sigma$ & $\mathrm{N}$ & {$[\mathrm{Ti} \mathrm{I} / \mathrm{Fe}]$} & $\sigma$ & $\mathrm{N}$ & {$[\mathrm{Ti} \mathrm{II} / \mathrm{Fe}]$} & $\sigma$ & $\mathrm{N}$ & {$[\mathrm{Sc}$ II $/ \mathrm{Fe}]$} & $\sigma$ & $\mathrm{N}$ & {$[\mathrm{Cr} \mathrm{I} / \mathrm{Fe}]$} & $\sigma$ & $\mathrm{N}$ & {$[\mathrm{Cr} \mathrm{II} / \mathrm{Fe}]$} & $\sigma$ & $\mathrm{N}$ \\
\hline \multicolumn{19}{|c|}{ RHB } \\
\hline HD 6229 & $\ldots$ & $\ldots$ & $\cdots$ & 0.07 & 0.08 & 13 & 0.34 & 0.14 & 10 & 0.34 & 0.12 & 4 & -0.15 & 0.08 & 5 & 0.03 & 0.14 & 5 \\
\hline HD 6461 & $\ldots$ & $\ldots$ & $\cdots$ & 0.19 & 0.10 & 13 & 0.43 & 0.10 & 9 & 0.35 & 0.11 & 4 & -0.11 & 0.05 & 2 & 0.10 & 0.20 & 5 \\
\hline HD 25532 & $\ldots$ & $\cdots$ & $\cdots$ & 0.18 & 0.07 & 8 & 0.22 & 0.09 & 7 & 0.12 & 0.06 & 2 & -0.21 & 0.12 & 4 & -0.08 & 0.17 & 5 \\
\hline HD 105546 & & $\ldots$ & $\ldots$ & 0.25 & 0.02 & 9 & 0.40 & 0.10 & 8 & 0.25 & 0.08 & 3 & -0.17 & 0.11 & 7 & 0.25 & 0.19 & 6 \\
\hline HD 119516 & -0.82 & $\ldots$ & 1 & 0.23 & 0.06 & 5 & 0.06 & 0.13 & 5 & -0.06 & & 1 & -0.18 & 0.06 & 5 & 0.01 & 0.10 & 5 \\
\hline $\mathrm{BD}+18^{\circ} 2890$ & & $\ldots$ & $\ldots$ & 0.15 & 0.09 & 6 & 0.00 & 0.08 & 3 & 0.06 & 0.09 & 2 & -0.17 & 0.01 & 2 & 0.26 & & 1 \\
\hline $\mathrm{BD}+11^{\circ} 2998$ & $\ldots$ & $\ldots$ & $\ldots$ & 0.19 & 0.04 & 10 & 0.22 & 0.12 & 6 & 0.16 & 0.05 & 3 & -0.22 & 0.08 & 4 & -0.05 & 0.12 & 3 \\
\hline $\mathrm{BD}+09^{\circ} 3223$ & & $\ldots$ & $\ldots$ & 0.28 & 0.08 & 8 & 0.16 & 0.09 & 9 & 0.06 & 0.02 & 3 & -0.21 & 0.07 & 4 & 0.12 & 0.18 & 2 \\
\hline $\mathrm{BD}+17^{\circ} 3248$ & & $\ldots$ & $\ldots$ & 0.28 & 0.06 & 6 & 0.26 & 0.09 & 8 & 0.16 & 0.07 & 2 & -0.27 & 0.08 & 5 & 0.25 & 0.09 & 4 \\
\hline HD 184266 & $\ldots$ & $\cdots$ & $\ldots$ & 0.30 & 0.07 & 6 & 0.21 & 0.10 & 5 & 0.09 & 0.02 & 3 & -0.06 & 0.06 & 3 & 0.14 & 0.17 & 5 \\
\hline HD 229274 & & $\ldots$ & $\ldots$ & 0.16 & 0.05 & 9 & 0.22 & 0.12 & 6 & 0.13 & 0.02 & 3 & -0.26 & 0.03 & 3 & 0.17 & 0.18 & 4 \\
\hline CS $22882-001$ & -0.77 & $\ldots$ & 1 & 0.55 & & 1 & 0.30 & 0.09 & 22 & 0.22 & 0.02 & 2 & -0.19 & & 1 & 0.39 & & 1 \\
\hline CS $22190-007$ & -0.80 & 0.17 & 2 & 0.37 & 0.10 & 4 & 0.17 & 0.08 & 23 & 0.06 & 0.13 & 4 & -0.11 & 0.16 & 6 & 0.25 & 0.04 & 2 \\
\hline CS 22186-005 & -0.82 & $\ldots$ & 1 & $\ldots$ & $\ldots$ & $\cdots$ & 0.03 & 0.04 & 6 & -0.01 & $\ldots$ & 1 & -0.15 & 0.11 & 4 & 0.76 & $\ldots$ & 1 \\
\hline CS $22191-029$ & -0.62 & 0.08 & 2 & 0.51 & 0.03 & 3 & 0.30 & 0.09 & 14 & 0.28 & 0.05 & 3 & -0.16 & 0.08 & 3 & 0.49 & $\ldots$ & 1 \\
\hline CS 22883-037 & -0.70 & $\ldots$ & 1 & 0.36 & $\ldots$ & 1 & 0.23 & 0.11 & 10 & 0.04 & 0.04 & 3 & -0.01 & 0.16 & 5 & 0.20 & 0.08 & 3 \\
\hline CS $22878-121$ & -0.88 & $\ldots$ & 1 & 0.34 & 0.11 & 6 & 0.21 & 0.10 & 27 & 0.15 & 0.09 & 6 & -0.09 & 0.12 & 9 & 0.20 & 0.12 & 4 \\
\hline CS $22891-184$ & -0.84 & 0.05 & 2 & 0.29 & 0.04 & 4 & 0.08 & 0.06 & 21 & -0.01 & 0.04 & 3 & -0.20 & 0.06 & 5 & 0.25 & 0.06 & 2 \\
\hline CS $22896-110$ & -0.46 & 0.21 & 2 & 0.45 & 0.08 & 5 & 0.19 & 0.11 & 17 & 0.06 & 0.01 & 3 & -0.14 & 0.14 & 6 & 0.48 & 0.11 & 2 \\
\hline CS $22940-077$ & -0.76 & $\ldots$ & 1 & 0.50 & 0.12 & 6 & 0.28 & 0.10 & 17 & 0.15 & 0.11 & 5 & -0.16 & 0.13 & 5 & 0.30 & & 1 \\
\hline CS $22955-174$ & -0.51 & $\ldots$ & 1 & 0.69 & 0.02 & 2 & 0.27 & 0.06 & 14 & 0.11 & 0.05 & 2 & -0.24 & 0.10 & 3 & 0.61 & 0.05 & 2 \\
\hline CS $22940-070$ & & $\ldots$ & $\ldots$ & 0.38 & 0.09 & 4 & 0.26 & 0.06 & 9 & 0.14 & 0.04 & 2 & 0.11 & & 1 & -0.01 & 0.16 & 2 \\
\hline CS $22879-103$ & -0.59 & 0.14 & 2 & 0.41 & 0.09 & 6 & 0.28 & 0.06 & 15 & 0.18 & 0.00 & 2 & -0.07 & 0.09 & 6 & -0.05 & 0.07 & 3 \\
\hline CS 22879-097 & -0.74 & $\ldots$ & 1 & 0.52 & 0.12 & 5 & 0.25 & 0.08 & 16 & 0.29 & 0.13 & 4 & -0.15 & 0.15 & 3 & 0.23 & 0.16 & 3 \\
\hline CS $22940-121$ & -0.48 & $\ldots$ & 1 & 0.43 & 0.13 & 3 & 0.27 & 0.10 & 15 & 0.19 & 0.12 & 3 & -0.19 & 0.13 & 4 & 0.14 & $\ldots$ & 1 \\
\hline CS 22898-043 & -0.72 & $\ldots$ & 1 & 0.47 & & 1 & 0.31 & 0.08 & 10 & 0.20 & & 1 & -0.12 & 0.11 & 2 & 0.45 & & 1 \\
\hline CS $22937-072$ & -0.49 & $\ldots$ & 1 & 0.43 & 0.09 & 9 & 0.23 & 0.09 & 20 & 0.11 & 0.05 & 4 & -0.22 & 0.07 & 3 & 0.50 & $\ldots$ & 1 \\
\hline CS 22948-006 & -0.72 & & 1 & 0.31 & 0.04 & 5 & 0.16 & 0.08 & 16 & -0.03 & 0.01 & 2 & -0.17 & 0.23 & 4 & 0.15 & 0.13 & 4 \\
\hline CS $22944-039$ & -0.68 & 0.16 & 2 & 0.28 & 0.14 & 3 & 0.10 & 0.11 & 19 & -0.14 & 0.08 & 3 & -0.17 & 0.05 & 4 & 0.00 & 0.06 & 4 \\
\hline CS $22951-077$ & -0.75 & 0.17 & 2 & 0.22 & 0.03 & 3 & 0.11 & 0.07 & 17 & -0.05 & 0.14 & 3 & -0.17 & 0.10 & 7 & 0.04 & 0.15 & 3 \\
\hline CS 22881-039 & -0.63 & 0.02 & 2 & 0.69 & & 1 & 0.24 & 0.08 & 15 & 0.20 & 0.05 & 2 & -0.20 & 0.11 & 4 & 0.25 & & 1 \\
\hline CS $22886-043$ & -0.58 & 0.14 & 2 & 0.45 & 0.05 & 3 & 0.38 & 0.13 & 6 & 0.29 & 0.18 & 2 & 0.03 & 0.13 & 6 & 0.02 & 0.11 & 2 \\
\hline CS 22875-029 & -0.42 & $\ldots$ & 1 & 0.63 & 0.01 & 3 & 0.33 & 0.08 & 18 & 0.30 & 0.10 & 3 & -0.11 & 0.08 & 3 & 0.37 & 0.11 & 3 \\
\hline CS $22888-047$ & -0.75 & 0.03 & 2 & 0.40 & 0.13 & 3 & 0.13 & 0.08 & 17 & 0.07 & 0.14 & 3 & -0.05 & 0.11 & 4 & 0.34 & 0.13 & 2 \\
\hline CS 22941-027 & -0.73 & 0.07 & 2 & 0.36 & $\ldots$ & 1 & 0.28 & 0.08 & 12 & $\ldots$ & $\ldots$ & $\ldots$ & -0.02 & 0.12 & 3 & 0.38 & 0.10 & 5 \\
\hline CS 22945-056 & -0.48 & & 1 & 0.79 & $\ldots$ & 1 & 0.19 & 0.06 & 8 & 0.18 & 0.04 & 3 & -0.13 & 0.06 & 3 & & & \\
\hline \multicolumn{19}{|c|}{ BHB } \\
\hline HD 2857 & $0.20^{\mathrm{a}}$ & $\cdots$ & 1 & $\ldots$ & $\ldots$ & $\therefore$ & 0.36 & 0.07 & 8 & 0.25 & 0.08 & 2 & 0.31 & $\cdots$ & 1 & -0.04 & 0.14 & 2 \\
\hline HD 8376 & & $\ldots$ & $\ldots$ & $\ldots$ & $\ldots$ & $\ldots$ & 0.43 & 0.07 & 11 & & 0.00 & & & & 工 & & & 2 \\
\hline HD 252940 & $\ldots$ & $\ldots$ & $\ldots$ & $\ldots$ & $\ldots$ & $\ldots$ & 0.36 & 0.07 & 8 & 0.07 & $\ldots$ & 1 & 0.07 & 0.06 & 2 & 0.14 & 0.02 & 2 \\
\hline HD 60778 & $\ldots$ & $\cdots$ & $\ldots$ & $\ldots$ & $\ldots$ & $\cdots$ & 0.27 & 0.12 & 11 & 0.10 & $\ldots$ & 1 & -0.17 & $\ldots$ & 1 & 0.17 & 0.06 & 2 \\
\hline HD 74721 & $\ldots$ & $\ldots$ & $\ldots$ & $\ldots$ & $\ldots$ & $\ldots$ & 0.28 & 0.09 & 11 & 0.08 & 0.05 & 2 & 0.02 & 0.06 & 4 & 0.03 & 0.15 & 7 \\
\hline HD 86986 & & $\ldots$ & $\ldots$ & $\ldots$ & $\ldots$ & & 0.34 & 0.05 & 12 & 0.15 & 0.04 & 2 & -0.04 & 0.12 & 5 & 0.15 & 0.12 & 7 \\
\hline HD 87047 & & $\ldots$ & $\ldots$ & $\ldots$ & $\ldots$ & $\ldots$ & 0.18 & 0.06 & 4 & 0.02 & & 1 & & & $\ldots$ & & & .. \\
\hline HD 93329 & $0.29^{\mathrm{a}}$ & $\ldots$ & 1 & $\ldots$ & $\ldots$ & $\ldots$ & 0.33 & 0.09 & 14 & 0.21 & 0.08 & 2 & 0.00 & 0.09 & 4 & 0.02 & 0.14 & 7 \\
\hline HD 109995 & $0.59^{\mathrm{a}}$ & $\ldots$ & 1 & $\ldots$ & $\ldots$ & $\ldots$ & 0.39 & 0.08 & 10 & 0.12 & $\ldots$ & 1 & & & $\ldots$ & 0.23 & 0.09 & 3 \\
\hline
\end{tabular}


Table 6 - Continued

\begin{tabular}{|c|c|c|c|c|c|c|c|c|c|c|c|c|c|c|c|c|c|c|}
\hline Star & {$[\mathrm{Al} \mathrm{I} / \mathrm{Fe}]$} & $\sigma$ & $\mathrm{N}$ & {$[\mathrm{Ti} \mathrm{I} / \mathrm{Fe}]$} & $\sigma$ & $\mathrm{N}$ & [Ti II/Fe] & $\sigma$ & $\mathrm{N}$ & {$[\mathrm{Sc} \mathrm{II} / \mathrm{Fe}]$} & $\sigma$ & $\mathrm{N}$ & {$[\mathrm{Cr} \mathrm{I} / \mathrm{Fe}]$} & $\sigma$ & $\mathrm{N}$ & {$[\mathrm{Cr} \mathrm{II} / \mathrm{Fe}]$} & $\sigma$ & $\mathrm{N}$ \\
\hline $\mathrm{BD}+25^{\circ} 2602$ & $\ldots$ & $\ldots$ & $\ldots$ & $\ldots$ & $\ldots$ & $\ldots$ & 0.28 & 0.07 & 8 & 0.19 & & 1 & & $\ldots$ & $\cdots$ & 0.51 & $\ldots$ & 1 \\
\hline HD 161817 & $\ldots$ & $\ldots$ & $\ldots$ & $\ldots$ & $\ldots$ & $\ldots$ & 0.35 & 0.13 & 25 & 0.21 & 0.03 & 3 & -0.08 & 0.09 & 3 & 0.04 & 0.14 & 8 \\
\hline HD 167105 & $\ldots$ & $\ldots$ & $\cdots$ & $\ldots$ & $\ldots$ & $\cdots$ & 0.17 & 0.05 & 6 & $\ldots$ & $\ldots$ & ... & $\ldots$ & $\ldots$ & $\ldots$ & 0.29 & 0.11 & 3 \\
\hline
\end{tabular}

${ }^{a}$ NLTE correction 
Table 7. Abundance ratios of Fe-peak elements: V, Mn, Co, Ni and Zn

\begin{tabular}{|c|c|c|c|c|c|c|c|c|c|c|c|c|c|c|c|c|c|c|}
\hline Star & {$[\mathrm{V}$ II $/ \mathrm{Fe}]$} & $\sigma$ & $\mathrm{N}$ & {$[\mathrm{Mn} \mathrm{I} / \mathrm{Fe}]$} & $\sigma$ & $\mathrm{N}$ & {$[\mathrm{Co} \mathrm{I} / \mathrm{Fe}]$} & $\sigma$ & $\mathrm{N}$ & {$[\mathrm{Ni} \mathrm{I} / \mathrm{Fe}]$} & $\sigma$ & $\mathrm{N}$ & {$[\mathrm{Ni}$ II/Fe] } & $\sigma$ & $\mathrm{N}$ & {$[\mathrm{Zn} \mathrm{I} / \mathrm{Fe}]$} & $\sigma$ & $\mathrm{N}$ \\
\hline \multicolumn{19}{|c|}{ RHB } \\
\hline HD 6229 & $\ldots$ & $\ldots$ & $\ldots$ & 0.12 & 0.27 & 3 & 0.80 & $\ldots$ & 1 & -0.04 & 0.09 & 9 & $\ldots$ & $\ldots$ & $\ldots$ & 0.11 & 0.04 & 2 \\
\hline HD 6461 & $\ldots$ & $\ldots$ & $\ldots$ & 0.30 & $\ldots$ & 1 & 0.84 & $\ldots$ & 1 & -0.01 & 0.1 & 9 & $\ldots$ & $\ldots$ & $\ldots$ & 0.24 & $\cdots$ & 1 \\
\hline HD 25532 & $\ldots$ & $\ldots$ & $\ldots$ & 0.05 & 0.07 & 3 & 0.37 & $\ldots$ & 1 & 0.05 & 0.12 & 4 & $\ldots$ & $\ldots$ & $\ldots$ & 0.04 & $\ldots$ & 1 \\
\hline HD 105546 & $\ldots$ & $\ldots$ & $\ldots$ & -0.09 & 0.16 & 5 & 0.30 & 0.08 & 2 & -0.03 & 0.13 & 5 & $\ldots$ & $\ldots$ & $\ldots$ & 0.13 & 0.05 & 2 \\
\hline HD 119516 & $\ldots$ & $\ldots$ & $\ldots$ & -0.30 & 0.08 & 3 & -0.01 & $\ldots$ & 1 & -0.04 & $\ldots$ & 1 & $\ldots$ & $\ldots$ & $\ldots$ & 0.05 & 0.04 & 2 \\
\hline $\mathrm{BD}+18^{\circ} 2890$ & $\ldots$ & & $\cdots$ & -0.70 & 0.08 & 3 & 0.22 & $\ldots$ & 1 & -0.03 & 0.09 & 3 & $\ldots$ & $\ldots$ & $\ldots$ & 0.04 & $\ldots$ & 1 \\
\hline $\mathrm{BD}+11^{\circ} 2998$ & $\ldots$ & $\ldots$ & $\ldots$ & -0.06 & 0.15 & 4 & 0.32 & 0.04 & 2 & 0.06 & 0.03 & 2 & $\ldots$ & $\ldots$ & $\ldots$ & $\ldots$ & $\ldots$ & $\ldots$ \\
\hline $\mathrm{BD}+09^{\circ} 3223$ & 0.03 & $\ldots$ & 1 & -0.10 & 0.11 & 4 & 0.42 & $\ldots$ & 1 & $\ldots$ & $\ldots$ & $\ldots$ & $\ldots$ & $\ldots$ & $\ldots$ & 0.20 & $\ldots$ & 1 \\
\hline $\mathrm{BD}+17^{\circ} 3248$ & & $\ldots$ & $\ldots$ & -0.18 & 0.08 & 4 & $\ldots$ & $\ldots$ & .. & $\ldots$ & $\ldots$ & $\ldots$ & $\ldots$ & $\ldots$ & $\ldots$ & 0.07 & 0.01 & 2 \\
\hline HD 184266 & 0.15 & $\ldots$ & 1 & -0.19 & 0.11 & 4 & -0.03 & $\ldots$ & 1 & 0.12 & $\ldots$ & 1 & $\ldots$ & $\ldots$ & $\ldots$ & & $\ldots$ & $\cdots$ \\
\hline HD 229274 & $\ldots$ & $\ldots$ & $\ldots$ & -0.06 & 0.24 & 4 & 0.34 & 0.15 & 2 & -0.03 & 0.11 & 6 & $\ldots$ & $\ldots$ & $\ldots$ & 0.01 & 0.01 & 2 \\
\hline CS 22882-001 & 0.31 & $\ldots$ & 1 & -0.39 & 0.05 & 3 & $\ldots$ & $\ldots$ & $\cdots$ & $\ldots$ & $\ldots$ & $\ldots$ & $\ldots$ & $\ldots$ & $\ldots$ & $\ldots$ & $\ldots$ & $\ldots$ \\
\hline CS $22190-007$ & 0.20 & & 1 & -0.50 & 0.02 & 3 & $\ldots$ & $\ldots$ & & $\ldots$ & $\ldots$ & & $\ldots$ & $\ldots$ & & $\ldots$ & $\ldots$ & $\ldots$ \\
\hline CS 22186-005 & & $\ldots$ & $\ldots$ & -0.46 & 0.05 & 3 & $\ldots$ & $\ldots$ & $\ldots$ & $\ldots$ & $\ldots$ & $\ldots$ & $\ldots$ & $\ldots$ & $\ldots$ & $\ldots$ & $\ldots$ & $\ldots$ \\
\hline CS $22191-029$ & 0.32 & $\ldots$ & 1 & -0.54 & 0.05 & 3 & $\ldots$ & $\ldots$ & $\ldots$ & $\ldots$ & $\ldots$ & $\ldots$ & $\ldots$ & $\ldots$ & $\ldots$ & $\ldots$ & $\ldots$ & $\ldots$ \\
\hline CS 22883-037 & -0.02 & $\ldots$ & 1 & -0.47 & 0.05 & 3 & $\ldots$ & $\ldots$ & $\ldots$ & $\ldots$ & $\ldots$ & $\ldots$ & $\ldots$ & $\ldots$ & $\ldots$ & 0.57 & $\ldots$ & 1 \\
\hline CS $22878-121$ & $\ldots$ & $\ldots$ & $\ldots$ & -0.33 & 0.17 & 3 & 0.44 & $\ldots$ & 1 & 0.41 & $\ldots$ & 1 & $\ldots$ & $\ldots$ & $\ldots$ & 0.10 & $\ldots$ & 1 \\
\hline CS $22891-184$ & $\ldots$ & $\ldots$ & $\ldots$ & -0.49 & 0.07 & 3 & $\ldots$ & $\ldots$ & $\ldots$ & . & $\ldots$ & $\ldots$ & $\ldots$ & $\ldots$ & $\ldots$ & $\cdots$ & $\cdots$ & $\ldots$ \\
\hline CS $22896-110$ & 0.13 & $\ldots$ & 1 & -0.45 & 0.09 & 3 & $\ldots$ & $\ldots$ & $\ldots$ & $\ldots$ & $\ldots$ & $\ldots$ & $\ldots$ & $\ldots$ & $\ldots$ & $\ldots$ & $\ldots$ & $\ldots$ \\
\hline CS $22940-077$ & $\ldots$ & $\ldots$ & $\ldots$ & -0.58 & 0.08 & 3 & $\ldots$ & $\ldots$ & $\ldots$ & $\ldots$ & $\ldots$ & $\ldots$ & $\ldots$ & $\ldots$ & $\ldots$ & $\ldots$ & $\ldots$ & $\ldots$ \\
\hline CS $22955-174$ & $\ldots$ & $\ldots$ & $\ldots$ & -0.63 & 0.02 & 3 & $\ldots$ & $\ldots$ & & $\ldots$ & $\ldots$ & $\ldots$ & $\ldots$ & $\ldots$ & $\ldots$ & 0.54 & $\ldots$ & 1 \\
\hline CS $22940-070$ & $\ldots$ & $\ldots$ & $\ldots$ & -0.37 & 0.05 & 3 & 0.50 & $\ldots$ & 1 & 0.69 & $\ldots$ & 1 & $\ldots$ & $\ldots$ & $\ldots$ & 0.06 & $\ldots$ & 1 \\
\hline CS $22879-103$ & $\ldots$ & $\ldots$ & $\ldots$ & -0.50 & 0.04 & 3 & $\ldots$ & $\ldots$ & $\ldots$ & $\ldots$ & $\ldots$ & $\ldots$ & $\ldots$ & $\ldots$ & $\ldots$ & 0.31 & $\ldots$ & 1 \\
\hline CS $22879-097$ & 0.13 & 0.02 & 2 & -0.58 & 0.05 & 3 & 0.78 & $\ldots$ & 1 & $\ldots$ & $\ldots$ & $\ldots$ & $\ldots$ & $\ldots$ & $\ldots$ & $\ldots$ & $\ldots$ & $\ldots$ \\
\hline CS $22940-121$ & 0.30 & 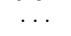 & 1 & -0.58 & 0.02 & 3 & $\ldots$ & $\ldots$ & $\ldots$ & $\ldots$ & $\ldots$ & $\ldots$ & $\ldots$ & $\ldots$ & $\ldots$ & $\ldots$ & $\ldots$ & $\ldots$ \\
\hline CS $22898-043$ & & $\ldots$ & & -0.30 & 0.05 & 3 & $\ldots$ & $\ldots$ & $\ldots$ & $\ldots$ & $\ldots$ & $\ldots$ & $\ldots$ & $\ldots$ & $\ldots$ & $\ldots$ & $\ldots$ & $\ldots$ \\
\hline CS $22937-072$ & 0.11 & 0.01 & 2 & -0.53 & 0.06 & 3 & $\ldots$ & $\ldots$ & $\ldots$ & $\ldots$ & $\ldots$ & $\ldots$ & $\ldots$ & $\ldots$ & $\ldots$ & $\ldots$ & $\ldots$ & $\ldots$ \\
\hline CS 22948-006 & 0.10 & $\ldots$ & 1 & -0.61 & 0.02 & 3 & $\ldots$ & $\ldots$ & & 0.59 & $\ldots$ & 1 & $\ldots$ & $\ldots$ & $\ldots$ & 0.42 & $\ldots$ & 1 \\
\hline CS 22944-039 & 0.05 & $\ldots$ & 1 & -0.45 & 0.04 & 3 & 0.35 & $\ldots$ & 1 & 0.43 & $\ldots$ & 1 & $\ldots$ & $\ldots$ & $\ldots$ & 0.10 & $\ldots$ & 1 \\
\hline CS 22951-077 & -0.04 & 0.02 & 2 & -0.33 & 0.17 & 3 & $\ldots$ & $\ldots$ & $\cdots$ & 0.39 & $\ldots$ & 1 & $\ldots$ & $\ldots$ & $\ldots$ & 0.19 & $\ldots$ & 1 \\
\hline CS 22881-039 & $\ldots$ & $\ldots$ & $\cdots$ & -0.37 & 0.02 & 3 & $\ldots$ & $\ldots$ & & $\ldots$ & $\ldots$ & & $\ldots$ & $\ldots$ & $\ldots$ & $\ldots$ & $\ldots$ & $\cdots$ \\
\hline CS $22886-043$ & $\ldots$ & $\ldots$ & $\ldots$ & -0.45 & 0.04 & 3 & 0.58 & $\ldots$ & 1 & 0.71 & $\ldots$ & 1 & $\ldots$ & $\ldots$ & $\ldots$ & 0.27 & $\ldots$ & 1 \\
\hline CS 22875-029 & 0.23 & $\ldots$ & 1 & -0.57 & 0.05 & 3 & $\ldots$ & $\ldots$ & $\ldots$ & $\ldots$ & $\ldots$ & $\ldots$ & $\ldots$ & $\ldots$ & $\ldots$ & $\ldots$ & $\ldots$ & $\ldots$ \\
\hline CS $22888-047$ & $\ldots$ & $\ldots$ & $\ldots$ & -0.57 & 0.08 & 3 & $\ldots$ & $\ldots$ & $\ldots$ & $\ldots$ & $\ldots$ & $\ldots$ & $\ldots$ & $\ldots$ & $\ldots$ & $\ldots$ & $\ldots$ & $\ldots$ \\
\hline CS 22941-027 & $\ldots$ & $\ldots$ & $\ldots$ & -0.36 & 0.04 & 3 & $\ldots$ & $\ldots$ & $\ldots$ & $\ldots$ & $\ldots$ & $\ldots$ & $\ldots$ & $\ldots$ & $\ldots$ & $\ldots$ & $\ldots$ & $\ldots$ \\
\hline CS 22945-056 & $\cdots$ & $\cdots$ & $\cdots$ & -0.51 & 0.05 & 3 & $\cdots$ & $\cdots$ & & $\cdots$ & $\cdots$ & $\cdots$ & $\cdots$ & $\cdots$ & $\cdots$ & $\cdots$ & $\cdots$ & $\cdots$ \\
\hline \multicolumn{19}{|c|}{ BHB } \\
\hline HD 2857 & $\ldots$ & $\ldots$ & $\ldots$ & $\ldots$ & $\ldots$ & $\ldots$ & $\ldots$ & $\ldots$ & $\ldots$ & $\ldots$ & $\ldots$ & $\ldots$ & $\ldots$ & $\ldots$ & $\ldots$ & $\ldots$ & $\ldots$ & $\ldots$ \\
\hline HD 8376 & $\ldots$ & $\ldots$ & & & $\ldots$ & $\cdots$ & $\ldots$ & $\ldots$ & & $\ldots$ & $\ldots$ & & $\ldots$ & $\ldots$ & $\ldots$ & $\ldots$ & $\cdots$ & $\ldots$ \\
\hline HD 252940 & $\ldots$ & $\ldots$ & $\ldots$ & $\ldots$ & $\ldots$ & $\ldots$ & $\ldots$ & $\ldots$ & $\ldots$ & $\ldots$ & $\ldots$ & $\ldots$ & $\ldots$ & $\ldots$ & $\ldots$ & $\ldots$ & $\ldots$ & $\ldots$ \\
\hline HD 60778 & 0.12 & $\ldots$ & 1 & $\ldots$ & $\ldots$ & $\ldots$ & $\ldots$ & $\ldots$ & $\ldots$ & $\ldots$ & $\ldots$ & $\ldots$ & -0.40 & $\ldots$ & 1 & $\ldots$ & $\ldots$ & $\ldots$ \\
\hline HD 74721 & 0.17 & 0.04 & 2 & $\ldots$ & $\ldots$ & $\ldots$ & $\ldots$ & $\ldots$ & $\ldots$ & $\ldots$ & $\ldots$ & $\ldots$ & -0.30 & $\ldots$ & 1 & $\ldots$ & $\ldots$ & $\ldots$ \\
\hline HD 86986 & 0.14 & 0.09 & 2 & 0.06 & 0.32 & 3 & $\ldots$ & $\ldots$ & $\ldots$ & $\ldots$ & $\ldots$ & $\ldots$ & $\ldots$ & $\ldots$ & $\ldots$ & $\ldots$ & $\ldots$ & $\cdots$ \\
\hline HD 87047 & $\ldots$ & $\ldots$ & $\cdots$ & $\ldots$ & $\ldots$ & $\cdots$ & $\ldots$ & $\cdots$ & $\ldots$ & $\ldots$ & $\ldots$ & $\cdots$ & $\ldots$ & $\ldots$ & $\ldots$ & $\ldots$ & $\cdots$ & $\cdots$ \\
\hline HD 93329 & 0.11 & 0.07 & 2 & -0.10 & 0.02 & 2 & $\ldots$ & $\ldots$ & $\ldots$ & $\ldots$ & $\ldots$ & $\ldots$ & -0.35 & $\ldots$ & 1 & $\ldots$ & $\ldots$ & $\ldots$ \\
\hline HD 109995 & $\ldots$ & $\ldots$ & & $\ldots$ & $\ldots$ & $\cdots$ & $\ldots$ & $\ldots$ & & & $\ldots$ & $\cdots$ & $\ldots$ & $\cdots$ & $\ldots$ & $\cdots$ & $\cdots$ & $\cdots$ \\
\hline
\end{tabular}


Table 7 - Continued

\begin{tabular}{|c|c|c|c|c|c|c|c|c|c|c|c|c|c|c|c|c|c|c|}
\hline Star & {$[\mathrm{V}$ II/Fe] } & $\sigma$ & $\mathrm{N}$ & {$[\mathrm{Mn} \mathrm{I} / \mathrm{Fe}]$} & $\sigma$ & $\mathrm{N}$ & {$[\mathrm{Co} \mathrm{I} / \mathrm{Fe}]$} & $\sigma$ & $\mathrm{N}$ & {$[\mathrm{Ni} \mathrm{I} / \mathrm{Fe}]$} & $\sigma$ & $\mathrm{N}$ & {$[\mathrm{Ni} \mathrm{II} / \mathrm{Fe}]$} & $\sigma$ & $\mathrm{N}$ & {$[\mathrm{Zn} \mathrm{I} / \mathrm{Fe}]$} & $\sigma$ & $\mathrm{N}$ \\
\hline $\mathrm{BD}+25^{\circ} 2602$ & $\ldots$ & $\ldots$ & $\ldots$ & $\ldots$ & & & & $\ldots$ & $\ldots$ & $\ldots$ & $\ldots$ & $\ldots$ & & & $\ldots$ & $\ldots$ & & $\ldots$ \\
\hline HD 161817 & 0.21 & 0.06 & 2 & -0.33 & 0.10 & 3 & 0.28 & $\ldots$ & 1 & $\ldots$ & $\ldots$ & $\ldots$ & $\ldots$ & $\ldots$ & $\ldots$ & $\ldots$ & $\ldots$ & $\ldots$ \\
\hline HD 167105 & $\ldots$ & & 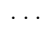 & $\ldots$ & & $\ldots$ & & $\ldots$ & $\ldots$ & .. & $\ldots$ & $\ldots$ & $\ldots$ & $\ldots$ & $\ldots$ & $\ldots$ & $\ldots$ & \\
\hline
\end{tabular}


Table 8. Abundance ratios of neutron-capture elements: Sr, Y, Zr, Ba, La and Eu

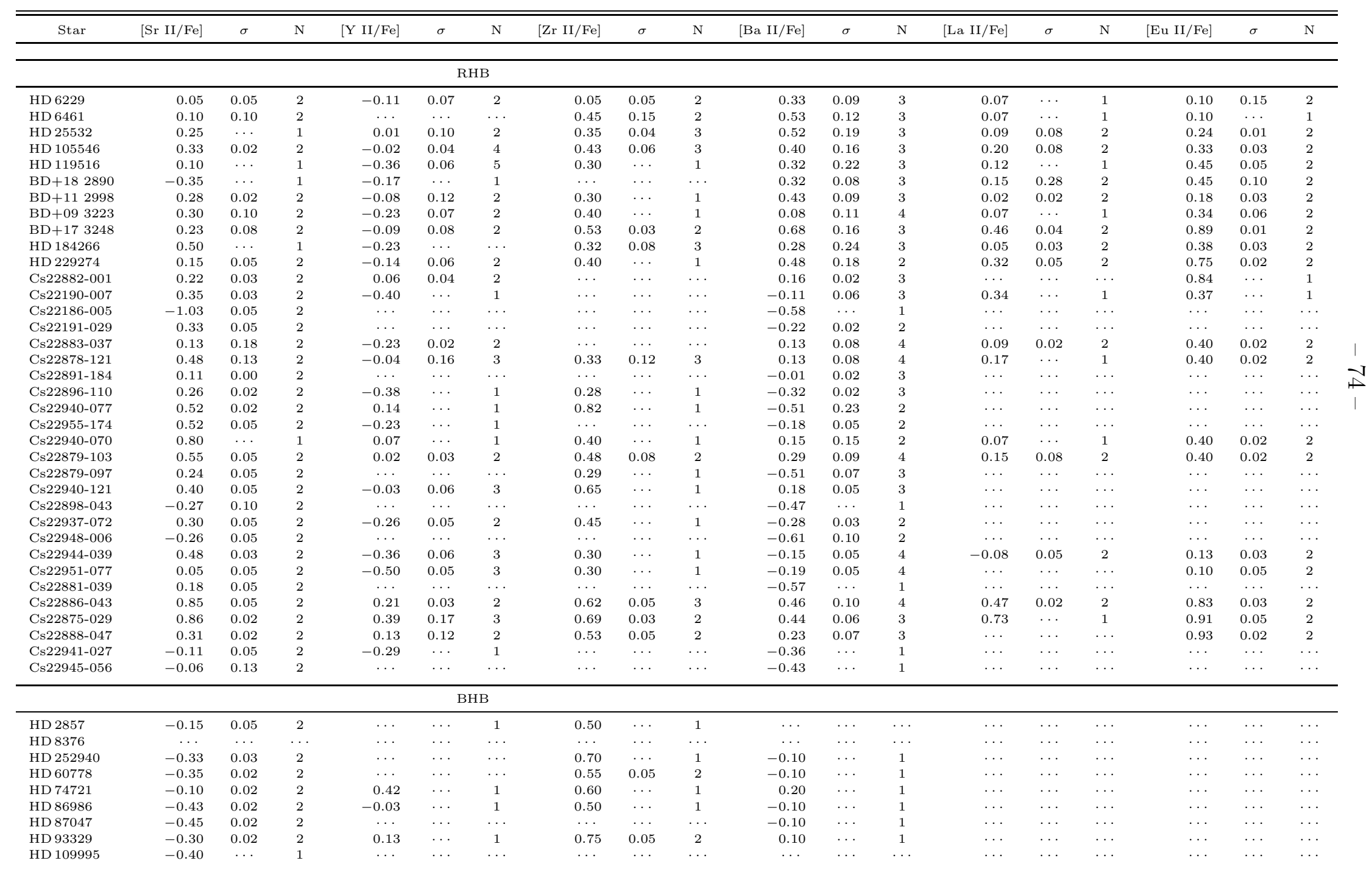


Table 8-Continued

\begin{tabular}{|c|c|c|c|c|c|c|c|c|c|c|c|c|c|c|c|c|c|c|}
\hline Star & {$[\mathrm{Sr}$ II/Fe] } & $\sigma$ & $\mathrm{N}$ & {$[\mathrm{Y}$ II $/ \mathrm{Fe}]$} & $\sigma$ & $\mathrm{N}$ & {$[\mathrm{Zr}$ II/Fe] } & $\sigma$ & $\mathrm{N}$ & {$[\mathrm{Ba} \mathrm{II} / \mathrm{Fe}]$} & $\sigma$ & $\mathrm{N}$ & {$[\mathrm{La} \mathrm{II} / \mathrm{Fe}]$} & $\sigma$ & $\mathrm{N}$ & [Eu II/Fe] & $\sigma$ & $\mathrm{N}$ \\
\hline $\mathrm{BD}+252602$ & -0.55 & & 1 & . . & $\ldots$ & $\ldots$ & $\ldots$ & $\ldots$ & $\ldots$ & & $\ldots$ & $\ldots$ & $\ldots$ & $\ldots$ & $\ldots$ & $\ldots$ & $\ldots$ & $\ldots$ \\
\hline HD 161817 & 0.02 & 0.08 & 2 & 0.36 & 0.01 & 2 & 0.65 & $\ldots$ & 1 & 0.08 & 0.03 & 2 & $\ldots$ & $\ldots$ & $\ldots$ & $\ldots$ & $\ldots$ & $\ldots$ \\
\hline HD 167105 & $\ldots$ & $\ldots$ & $\cdots$ & $\ldots$ & $\ldots$ & $\cdots$ & $\ldots$ & $\ldots$ & $\ldots$ & $\ldots$ & $\ldots$ & $\ldots$ & $\ldots$ & $\ldots$ & $\ldots$ & $\ldots$ & $\ldots$ & $\ldots$ \\
\hline
\end{tabular}


Table 9. Mean abundance ratios of various elements.

\begin{tabular}{lrccr}
\hline \hline Element & RHB & $N$ & BHB & \multicolumn{1}{c}{$N$} \\
\hline Na I & 0.37 & 27 & -0.45 & 2 \\
Mg I & 0.47 & 36 & 0.36 & 12 \\
Al I & -0.67 & 25 & 0.36 & 3 \\
Si I & 0.35 & 36 & -0.03 & 12 \\
Si II & 0.59 & 35 & 0.21 & 12 \\
Ca I & 0.37 & 36 & 0.07 & 12 \\
Ca II & $\ldots$ & $\ldots$ & 0.18 & 12 \\
Sc II & 0.13 & 35 & 0.14 & 10 \\
Ti I & 0.37 & 35 & $\ldots$ & $\ldots$ \\
Ti II & 0.23 & 36 & 0.31 & 12 \\
V II & 0.14 & 14 & 0.15 & 5 \\
Cr I & -0.14 & 36 & 0.02 & 7 \\
Cr II & 0.23 & 35 & 0.15 & 10 \\
Mn I & -0.37 & 36 & -0.13 & 3 \\
Co I & 0.41 & 15 & 0.28 & 1 \\
Ni I & 0.22 & 15 & $\ldots$ & $\ldots$ \\
Ni II & $\ldots$ & $\ldots$ & -0.35 & 3 \\
Zn I & 0.19 & 18 & $\ldots$ & $\ldots$ \\
Sr II & 0.23 & 36 & -0.30 & 10 \\
Y II & -0.12 & 27 & 0.22 & 4 \\
Zr II & 0.42 & 23 & 0.61 & 7 \\
Ba II & 0.03 & 36 & 0.00 & 7 \\
La II & 0.19 & 19 & $\ldots$ & $\ldots$ \\
Eu II & 0.45 & 22 & $\ldots$ & $\ldots$ \\
\hline & & & &
\end{tabular}


Table 10. Sensitivity of $[\mathrm{X} / \mathrm{Fe}]$ with stellar parameters.

\begin{tabular}{|c|c|c|c|c|}
\hline Stellar Parameters & $\begin{array}{l}\text { Species } \\
\Delta[\mathrm{X} / \mathrm{Fe}]\end{array}$ & CS 22898-043 & $\begin{array}{c}\text { Star } \\
\text { HS } 25532\end{array}$ & $\mathrm{BD}+18^{\circ} 2890$ \\
\hline$T_{\text {eff }}+150$ & $\mathrm{Na} \mathrm{I}$ & $\ldots$ & +0.16 & +0.16 \\
\hline$(\mathrm{K})$ & $\mathrm{Mg} \mathrm{I}$ & +0.09 & +0.08 & +0.25 \\
\hline $\log g+0.15$ & $\mathrm{NaI}$ & $\ldots$ & -0.05 & -0.03 \\
\hline$(\operatorname{dex})$ & Mg I & +0.01 & -0.02 & -0.01 \\
\hline$[\mathrm{M} / \mathrm{H}]+0.1$ & $\mathrm{NaI}$ & $\ldots$ & -0.01 & +0.00 \\
\hline$(\mathrm{dex})$ & $\mathrm{Mg} \mathrm{I}$ & $\ldots$ & -0.01 & -0.01 \\
\hline$v_{\mathrm{t}}+0.2$ & $\mathrm{Na} \mathrm{I}$ & $\ldots$ & -0.01 & -0.05 \\
\hline$\left(\mathrm{km} \mathrm{s}^{-1}\right)$ & $\mathrm{Mg} \mathrm{I}$ & -0.05 & -0.10 & -0.07 \\
\hline
\end{tabular}

Note. - Table 10 is published in its entirety in the electronic edition of the Astronomical Journal. A portion is shown here for guidance regarding its form and content. 
Table 11. Sensitivity of $[\mathrm{X} / \mathrm{Fe}]$ with stellar parameters for BHB star.

\begin{tabular}{llc}
\hline \hline Stellar Parameters & Species & $\begin{array}{c}\text { Star } \\
\end{array}$ \\
& $\Delta[\mathrm{X} / \mathrm{Fe}]$ & HD 93329 \\
\hline$T_{\text {eff }}+200$ & $\mathrm{Na} \mathrm{I}$ & +0.18 \\
$(\mathrm{~K})$ & $\mathrm{Mg} \mathrm{I}$ & +0.14 \\
$\log g+0.15$ & $\mathrm{Na} \mathrm{I}$ & -0.03 \\
$(\mathrm{dex})$ & $\mathrm{Mg} \mathrm{I}$ & -0.04 \\
{$[\mathrm{M} / \mathrm{H}]+0.1$} & $\mathrm{Na} \mathrm{I}$ & +0.01 \\
$(\mathrm{dex})$ & $\mathrm{Mg} \mathrm{I}$ & +0.00 \\
$v_{\mathrm{t}}+0.2$ & $\mathrm{Na} \mathrm{I}$ & -0.02 \\
$\left(\mathrm{~km} \mathrm{~s}^{-1}\right)$ & $\mathrm{Mg} \mathrm{I}$ & -0.01 \\
\hline
\end{tabular}

Note. - Table 11 is published in its entirety in the electronic edition of the Astronomical Journal. A portion is shown here for guidance regarding its form and content. 
Table 12. Comparison of HB model

\begin{tabular}{ccccc}
\hline \hline Model & $\begin{array}{c}\text { Mass } \\
\left(M / M_{\odot}\right)\end{array}$ & $\begin{array}{c}\log T_{\text {eff }} \\
(\mathrm{K})\end{array}$ & $\Delta \log g^{\mathrm{a}}$ & $\Delta \log L^{\mathrm{a}}$ \\
\hline Lee \& Demarque $(1990)$ & 0.56 & 4.22 & +0.02 & -0.02 \\
Lee \& Demarque $(1990)$ & 0.56 & 4.26 & +0.11 & -0.11 \\
Lee \& Demarque $(1990)$ & 0.78 & 3.86 & -0.01 & +0.01 \\
Lee \& Demarque $(1990)$ & 0.78 & 3.72 & +0.09 & -0.09 \\
\hline
\end{tabular}

a Pietrinferni et al. (2006) minus Lee \& Demarque (1990) model 
Table 13. Estimated HB masses and Parameters Used

\begin{tabular}{|c|c|c|c|c|}
\hline Stars & $\begin{array}{c}T_{\text {eff }, \text { spec }} \\
(\mathrm{K})\end{array}$ & $\begin{array}{l}\log g \\
(\operatorname{dex})\end{array}$ & $\begin{array}{c}{[\mathrm{Fe} / \mathrm{H}]} \\
(\mathrm{dex})\end{array}$ & $\begin{array}{c}\text { Mass } \\
M_{\odot}\end{array}$ \\
\hline \multicolumn{5}{|c|}{ RHB } \\
\hline HD 6229 & 5200 & $2.86^{\mathrm{a}}$ & -1.07 & 0.80 \\
\hline HD 6461 & 5200 & $3.26^{\mathrm{a}}$ & -0.75 & 0.80 \\
\hline HD 25532 & 5450 & $2.20^{\mathrm{a}}$ & -1.41 & 0.60 \\
\hline HD 105546 & 5200 & $2.66^{\mathrm{a}}$ & -1.54 & 0.80 \\
\hline HD 119516 & 5400 & $1.73^{\mathrm{a}}$ & -2.16 & 0.54 \\
\hline $\mathrm{BD}+18^{\circ} 2890$ & 5000 & $2.89^{\mathrm{a}}$ & -1.61 & 0.80 \\
\hline $\mathrm{BD}+11^{\circ} 2998$ & 5450 & $2.50^{\mathrm{a}}$ & -1.28 & 0.72 \\
\hline $\mathrm{BD}+09^{\circ} 3223$ & 5100 & $1.72^{\mathrm{a}}$ & -2.47 & 0.61 \\
\hline $\mathrm{BD}+17^{\circ} 3248$ & 5100 & $2.12^{\mathrm{a}}$ & -2.24 & 0.80 \\
\hline HD 184266 & 5700 & $1.75^{\mathrm{a}}$ & -1.79 & 0.52 \\
\hline HD 229274 & 5500 & $2.47^{\mathrm{a}}$ & -1.41 & 0.73 \\
\hline CS 22882-001 & 5950 & $1.91^{\mathrm{a}}$ & -2.54 & 0.54 \\
\hline CS 22190-007 & 5600 & $2.01^{\mathrm{a}}$ & -2.67 & 0.58 \\
\hline CS 22186-005 & 6200 & $2.22^{\mathrm{a}}$ & -2.77 & 0.57 \\
\hline CS 22191-029 & 6000 & $1.98^{\mathrm{a}}$ & -2.73 & 0.55 \\
\hline CS 22883-037 & 5900 & $1.59^{\mathrm{a}}$ & -1.95 & 0.52 \\
\hline CS 22878-121 & 5450 & $1.95^{\mathrm{a}}$ & -2.38 & 0.57 \\
\hline CS 22891-184 & 5600 & $1.81^{\mathrm{a}}$ & -2.61 & 0.54 \\
\hline CS 22896-110 & 5400 & $1.68^{\mathrm{a}}$ & -2.78 & 0.54 \\
\hline CS 22940-077 & 5300 & $1.74^{\mathrm{a}}$ & -3.02 & 0.56 \\
\hline CS 22955-174 & 5350 & $1.61^{\mathrm{a}}$ & -3.17 & 0.54 \\
\hline CS 22940-070 & 6300 & $2.12^{\mathrm{a}}$ & -1.41 & 0.53 \\
\hline CS 22879-103 & 5700 & $1.65^{\mathrm{a}}$ & -2.20 & 0.52 \\
\hline CS 22879-097 & 5650 & $2.03^{\mathrm{a}}$ & -2.59 & 0.57 \\
\hline CS 22940-121 & 5350 & $1.86^{\mathrm{a}}$ & -2.95 & 0.57 \\
\hline CS 22898-043 & 5900 & $1.94^{\mathrm{a}}$ & -3.03 & 0.55 \\
\hline CS 22937-072 & 5300 & $1.79^{\mathrm{a}}$ & -2.85 & 0.57 \\
\hline CS 22948-006 & 5400 & $1.63^{\mathrm{a}}$ & -2.79 & 0.54 \\
\hline CS 22944-039 & 5350 & $1.46^{\mathrm{a}}$ & -2.43 & 0.52 \\
\hline CS 22951-077 & 5350 & $1.81^{\mathrm{a}}$ & -2.44 & 0.56 \\
\hline CS 22881-039 & 6100 & $1.68^{\mathrm{a}}$ & -2.73 & 0.53 \\
\hline CS 22886-043 & 6000 & $1.73^{\mathrm{a}}$ & -2.17 & 0.52 \\
\hline CS 22875-029 & 6000 & $1.93^{\mathrm{a}}$ & -2.66 & 0.54 \\
\hline CS 22888-047 & 5850 & $1.66^{\mathrm{a}}$ & -2.58 & 0.53 \\
\hline CS 22941-027 & 6200 & $1.97^{\mathrm{a}}$ & -2.54 & 0.54 \\
\hline CS 22945-056 & 5850 & $1.46^{\mathrm{a}}$ & -2.92 & 0.52 \\
\hline \multicolumn{5}{|c|}{ BHB } \\
\hline HD 2857 & 8100 & $2.48^{\mathrm{b}}$ & -1.39 & 0.52 \\
\hline HD 8376 & 8600 & $2.38^{\mathrm{b}}$ & -2.39 & 0.52 \\
\hline HD 252940 & 7650 & $1.77^{\mathrm{b}}$ & -1.69 & 0.56 \\
\hline HD 60778 & 8100 & $1.63^{\mathrm{b}}$ & -1.43 & 0.54 \\
\hline HD 74721 & 9000 & $1.93^{\mathrm{b}}$ & -1.23 & 0.59 \\
\hline HD 86986 & 8200 & $2.04^{\mathrm{b}}$ & -1.61 & 0.63 \\
\hline
\end{tabular}


Table 13-Continued

\begin{tabular}{lcccc}
\hline \hline \multicolumn{1}{c}{ Stars } & $\begin{array}{c}T_{\text {eff,spec }} \\
(\mathrm{K})\end{array}$ & $\begin{array}{c}\log g \\
(\mathrm{dex})\end{array}$ & $\begin{array}{c}{[\mathrm{Fe} / \mathrm{H}]} \\
(\mathrm{dex})\end{array}$ & $\begin{array}{c}\text { Mass } \\
M_{\odot}\end{array}$ \\
\hline HD 87047 & 7700 & $1.35^{\mathrm{b}}$ & -2.38 & 0.53 \\
HD 93329 & 8700 & $2.04^{\mathrm{b}}$ & -1.10 & 0.59 \\
HD 109995 & 8600 & $1.68^{\mathrm{b}}$ & -1.60 & 0.56 \\
BD +25 2602 & 8400 & $1.56^{\mathrm{b}}$ & -1.98 & 0.55 \\
HD 161817 & 7800 & $2.01^{\mathrm{b}}$ & -1.43 & 0.59 \\
HD 167105 & 9000 & $1.63^{\mathrm{b}}$ & -1.55 & 0.56 \\
\hline
\end{tabular}

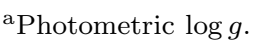

${ }^{\mathrm{b}}$ Spectroscopic $\log g$. 


\section{Table 14. Data Sources}

\begin{tabular}{ll}
\hline \hline \multicolumn{1}{c}{ References } \\
\hline Venn et al. (2004) & \multicolumn{1}{c}{ Element } \\
\hline Cohen et al. (2004) & $\mathrm{Ni}, \mathrm{Mg}, \mathrm{Ca}, \mathrm{Tr}, \mathrm{Tr}, \mathrm{Ni}, \mathrm{Y}, \mathrm{Ba}, \mathrm{La}, \mathrm{Mu}$ \\
Lai et al. (2008) & $\mathrm{Si}, \mathrm{Al}, \mathrm{Sc}, \mathrm{V}, \mathrm{Mn}, \mathrm{Zn}, \mathrm{Sr}, \mathrm{Zr}$ \\
Fulbright (2000) & $\mathrm{Si}, \mathrm{Al}, \mathrm{Cr}, \mathrm{V}, \mathrm{Zr}$ \\
Reddy et al. (2003) & $\mathrm{Al}, \mathrm{Sc}, \mathrm{Cr}, \mathrm{V}, \mathrm{Mn}, \mathrm{Ni}, \mathrm{Zn}$ \\
Sobeck et al. (2006) & $\mathrm{Mn}$ \\
Cavrel et al. (2004) & $\mathrm{Si}, \mathrm{Zn}$ \\
Stephens \& Boesgaard (2002) & $\mathrm{Si}, \mathrm{Ni}$ \\
Nissen et al. (2007) & $\mathrm{Ni}$ \\
\hline
\end{tabular}

